\title{
WestVirginiaUniversity
}

THE RESEARCH REPOSITORY @ WVU

Graduate Theses, Dissertations, and Problem Reports

2014

\section{Plasmonic Gold Nanohole Arrays for Surface-Enhanced Raman Scattering}

Peng Zheng

Follow this and additional works at: https://researchrepository.wvu.edu/etd

\section{Recommended Citation}

Zheng, Peng, "Plasmonic Gold Nanohole Arrays for Surface-Enhanced Raman Scattering" (2014). Graduate Theses, Dissertations, and Problem Reports. 7043.

https://researchrepository.wvu.edu/etd/7043

This Thesis is protected by copyright and/or related rights. It has been brought to you by the The Research Repository @ WVU with permission from the rights-holder(s). You are free to use this Thesis in any way that is permitted by the copyright and related rights legislation that applies to your use. For other uses you must obtain permission from the rights-holder(s) directly, unless additional rights are indicated by a Creative Commons license in the record and/ or on the work itself. This Thesis has been accepted for inclusion in WVU Graduate Theses, Dissertations, and Problem Reports collection by an authorized administrator of The Research Repository @ WVU. For more information, please contact researchrepository@mail.wvu.edu. 
Plasmonic Gold Nanohole Arrays for Surface-Enhanced Raman Scattering

\author{
Peng Zheng
}

\author{
Thesis submitted \\ to the Benjamin M. Statler College of Engineering and Mineral Resources \\ at West Virginia University \\ in partial fulfillment of the requirements for the degree of \\ Master of Science in \\ Mechanical Engineering
}
Nianqiang Wu, Ph.D., Chair, Advisor
Yuxin Liu, Ph.D., Co-advisor
Ever J. Barbero, Ph.D.
Yon Rojanasakul, Ph.D.

Department of Mechanical and Aerospace Engineering

Morgantown, West Virginia

2014

Keywords: Plasmon, Gold Nanohole Array, Surface-Enhanced Raman Scattering, Sensor Copyright 2014, Peng Zheng 


\section{Abstract \\ Plasmonic Gold Nanohole Arrays for Surface-Enhanced Raman Scattering \\ Peng Zheng}

Localized and periodic optical modes co-exist in plasmonic nanohole arrays leading to a unique mix of optical properties. In this thesis, a simultaneous experimental and theoretical investigation is used to investigate the origin of each optical mode, explaining the mix between extraordinary optical transmission, enhanced absorption, and local electromagnetic field concentration. Additionally, the hole array diameter, film thickness, and periodicity are systematically varied to explore the tuning and cross over between the multiple optical modes. The nanohole array to nanotriangle array transition is investigated, showing how the localized surface plasmon resonance evolves into the periodic surface plasmon polariton mode. These effects are correlated to the SERS intensity, revealing the relative amount that defects, the local field strength, and the reflectance combine to modulate performance.

Optimization of the SERS and optical related parameters is used to create a facile, ultrasensitive, highly selective, and reproducible sensor for silver ion detection based on Au nanohole arrays and Au nanostar@MGITC@ $\mathrm{SiO}_{2}$ sandwich nanostructure. In the presence of silver ions, $\mathrm{C}-\mathrm{Ag}^{+}-$ $\mathrm{C}$ mismatches hybridize to bring single stranded DNA attached to the Au nanohole array and $\mathrm{Au}$ nanostar@MGITC@SiO 2 together. This hybridizes the local electromagnetic field between the two plasmonic nanostructures, increasing the SERS intensity, and allowing a limit of detection of $170 \mathrm{fM}$. The complimentary DNA sequence lends to excellent stability and anti-interference. The design parameters, physical origin, and sensor design strategy developed in this thesis will guide the future development of plasmonic light enhancement architectures and SERS based sensors. 


\section{Acknowledgement}

First and foremost, my sincere gratitude goes to my advisor Professor Nianqiang Wu for his continuous support and guidance of my Master thesis, for his motivation, patience, enthusiasm, and profound knowledge since I joined the group in August, 2011.

Besides my advisor, I would like to express my sincere thanks to my Master's thesis committee: Professor Yuxin Liu, Professor Ever J. Barbero, and Professor Yon Rojanasakul for their valuable time, encouragement, and insightful advice.

My sincere thanks also go to all my teammates here at West Virginia University: Dr. Ming Li, Scott Cushing, Dr. Rui Li, Dr. Jiangtian Li, Dr. Honglei Gou, Dr. Mingjia Zhi, Dr. Fanke Meng, Jinlong Yan, Chengchen Xiang, Zhenhuan Zhao, Israa A Zaidan, Savan Suri, Anveeksh Koneru, Shimeng Hao, Joseph Bright, and Yang He. Were it not for their support and encouragement, I could not have had enjoyed so much progress and so much fun during the past three years.

I would also like to express my sincere thanks to Dr. Kolin Brown, Dr. Weiqiang Ding, Dr. Marcela Redigola, Dr. Harley Hart, and Dr. Huiyuan Li for technique supports in my research.

My special thanks along with my deepest love go to a special person, my girlfriend Xiaorui Tong. Since 2007 when I was so fortunate to meet her, she has always been standing as the person in my life who gives me the most understandings, shows me the strongest support, and trusts me most unconditionally whatever I am pursuing.

Last but not the least, I would like to express my sincere thanks and love to my family: my aging parents and my married elder sister. They are the most ignored persons in my life during my 
schooling years but they are also the very persons who offer me the strongest sense of belongings, security, and hope however far away I am staying from home.

\section{Financial source support}

Research reported in this publication was supported by the National Institute of Neurological Disorders And Stroke of the National Institutes of Health under Award Number R15NS087515.

The content is solely the responsibility of the authors and does not necessarily represent the official views of the National Institutes of Health. I am also grateful to the partial support by the National Science Foundation Grant (CBET-1336205).

We also acknowledge use of the WVU Shared Research Facilities. 


\section{Table of Contents}

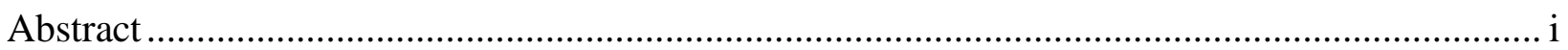

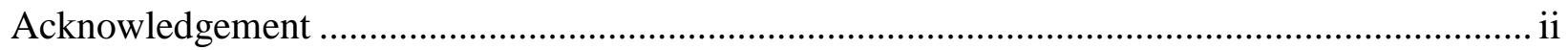

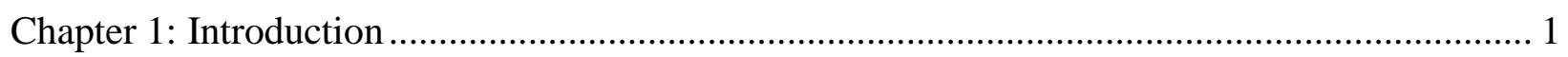

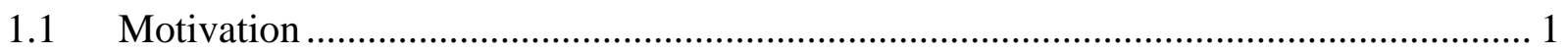

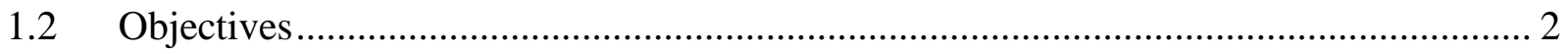

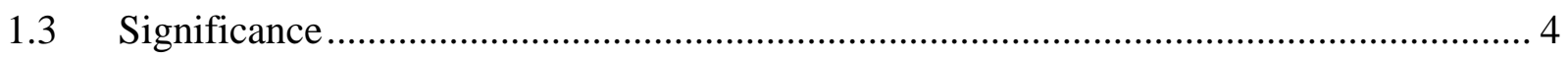

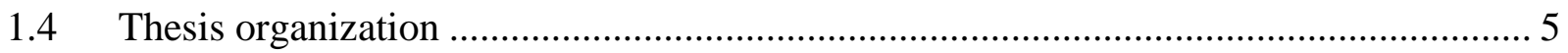

Chapter 2 Background and Literature Review..................................................................... 6

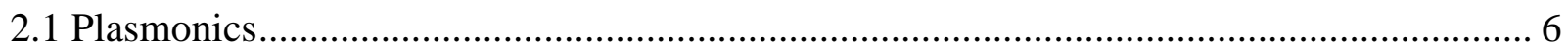

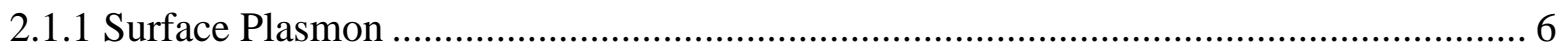

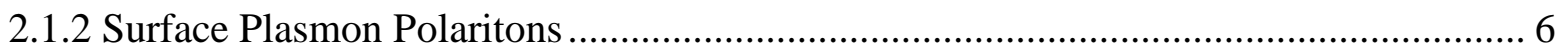

2.1.3 Localized Surface Plasmon Resonance ................................................................... 10

2.2 Optical Properties of Gold Nanohole Arrays .............................................................. 12

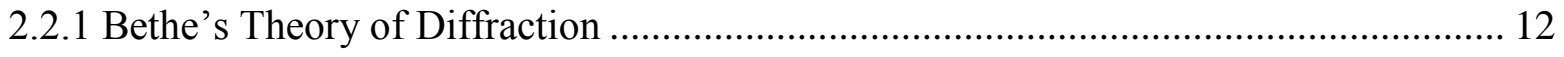

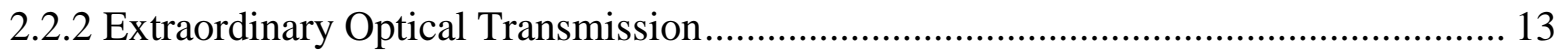


Chapter 3: Optical Studies of the Transition from Gold Nanotriangle Arrays to Gold Nanohole

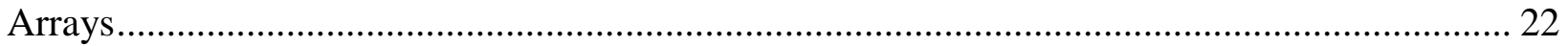

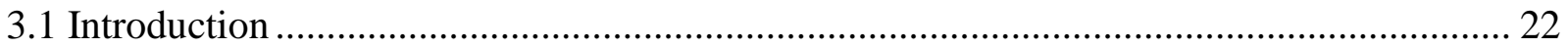

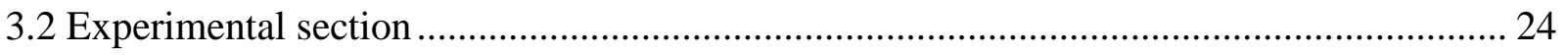

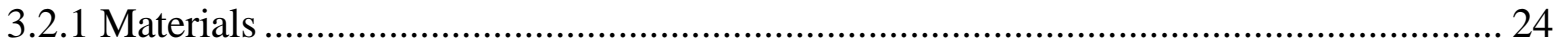

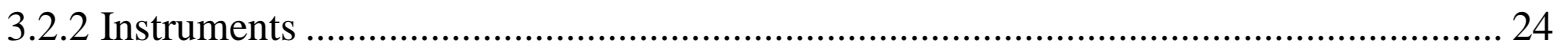

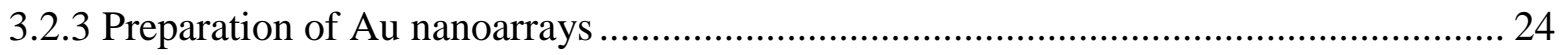

3.2.4 Measuring the refractive index sensitivity of Au nanoarrays....................................... 27



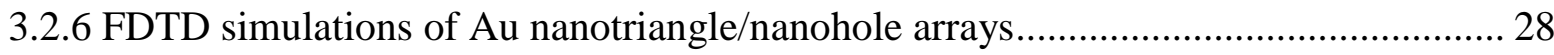

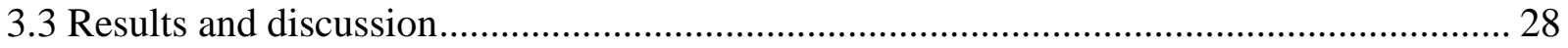

3.3.1 Angle-resolved optical study ................................................................................. 29

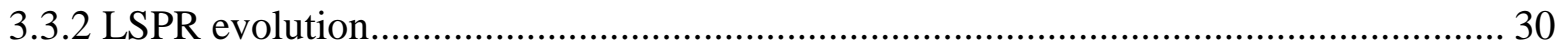

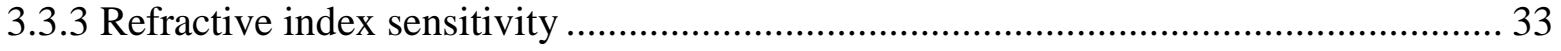

3.3.4 Hole size dependent resonance modes .................................................................... 34 
3.3.5 Au film thickness dependent resonance modes 36

3.3.6 Periodicity dependent resonance modes 38

3.3.7 SERS response of Au nanoarrays 39

3.3.8 Scale Invariant Properties 44

3.4 Conclusion 44

Chapter 4: Gold Nanohole Arrays Based Surface-Enhanced Raman Scattering (SERS) Biosensor

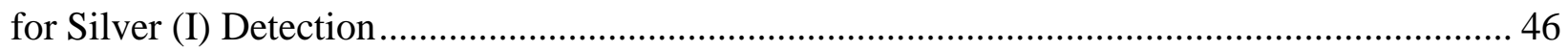

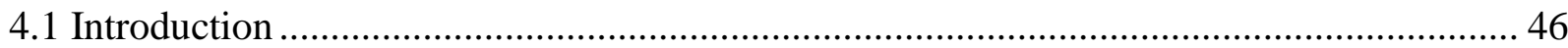

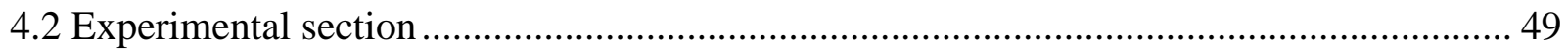

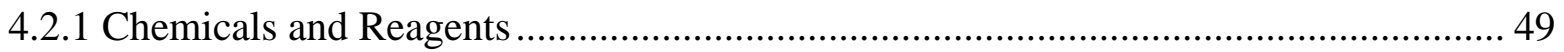

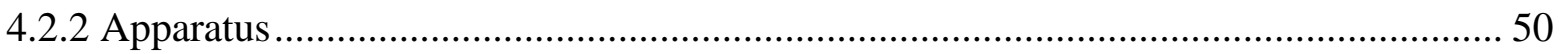

4.2.3 Preparation of Au nanostar@MGITC@SiO2 sandwiched nanoparticles ................... 50

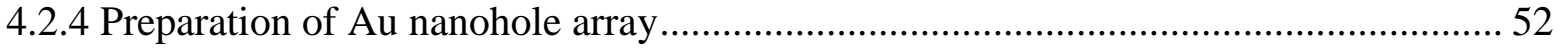

4.2.5 DNA Functionalization of Au Nanostar@MGITC@ $\mathrm{SiO}_{2}{ }^{15}$..................................... 53

4.2.6 Functionalization of Au Nanohole array/Au film............................................... 53

4.2.7 Preparation of Metal Ions Solutions .................................................................. 54




4.3.1 Operation Mechanism of SERS Sensor.

4.3.2 Comparison of SERS Sensor Performance on Au Nanohole Array and Au film ........ 56

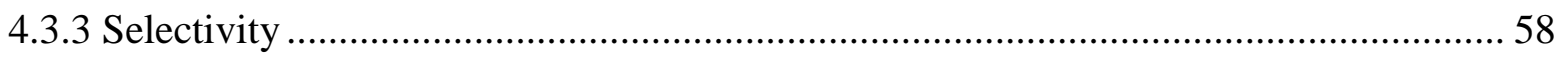

4.3.4 SERS Enhancement by Au Nanohole array ...................................................... 59

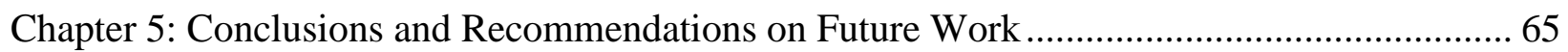

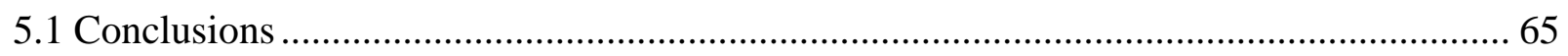

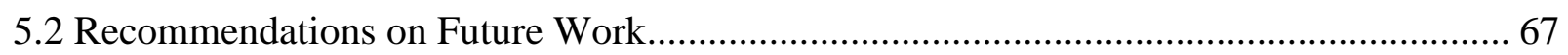

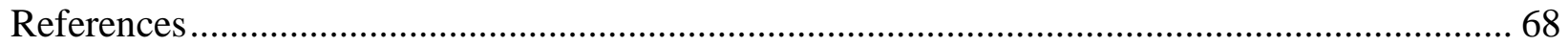

Peer-reviewed Publication List ........................................................................................ 79 


\section{List of Figures}

\section{Chapter Two}

Figure 2.1 Schematic illustration of the surface plasmon polaritions at the interface between the dielectric and the metallic surface ${ }^{21}$ 8

Figure 2.2 Shown in red is the dispersion relation of surface plasmon polaritons on the dielectricmetallic interface. Also shown are the surface plasmon frequency, bulk plasma frequency, the light line in air, and the light line in dielectric.

Figure 2.3 Schematic illustration of the dipolar excitation of the localized surface plasmon

resonance (LSPR) of metallic nanoparticle ${ }^{25}$ 11

Figure 2.4 Scheme of mechanism of SPR Sensor: ligand capturing the analyte on nanohole

arrays, shifting the SPR peak.

Figure 2.5 The sensorgram shows a cycle of SPR sensing process 16

\section{Chapter Three}

Figure 3.1 Protocol of nanosphere lithography for Au nanohole arrays fabrication. (a) Substrate (quartz slides) cleaning using acid piranha at $90{ }^{\circ} \mathrm{C}$ for two hours and sonicated in acetone, methanol, and D.I. water; (b) a monolayer of polystyrene was pattern on the cleaned substrate; (c) etch the PS spheres using Oxygen Plasma Asher; (d) a layer of $5 \mathrm{~nm}$ Ti was deposited; (e) a layer of $45 \mathrm{~nm}$ Au was deposited (f) PS spheres were removed by sonication in methanol; (g) schematic Au nanohole arrays. 
Figure 3.2 (a) to (h) corresponds to Au nanoarrays with a periodicity of $500 \mathrm{~nm}$ and hole diameters of $500 \mathrm{~nm}, 470 \mathrm{~nm}, 440 \mathrm{~nm}, 410 \mathrm{~nm}, 380 \mathrm{~nm}, 350 \mathrm{~nm}, 320 \mathrm{~nm}, 290 \mathrm{~nm}$. (i) Hole diameter decreases linearly with etching time 26

Figure 3.3 (a) to (h) corresponds to Au nanoarrays with a periodicity of $600 \mathrm{~nm}$ and hole diameters of $600 \mathrm{~nm}, 570 \mathrm{~nm}, 540 \mathrm{~nm}, 510 \mathrm{~nm}, 480 \mathrm{~nm}, 450 \mathrm{~nm}, 420 \mathrm{~nm}, 390 \mathrm{~nm}$. (i) Hole diameter decreases linearly with etching time.

Figure 3.4 Simulated angle-resolved transmission \& reflection spectra for Au nanohole arrays with a periodicity of $500 \mathrm{~nm}$ ((a) and (b)), hole diameter of $350 \mathrm{~nm}$, and film thickness of $50 \mathrm{~nm}$, and $600 \mathrm{~nm}((\mathrm{c})$ and (d)), hole diameter of $420 \mathrm{~nm}$, and film thickness of $50 \mathrm{~nm}$. The incident angle is defined as the angle between the incident light and the direction normal to the surface. 30

Figure 3.5 Experimental UV-Vis spectra for Au nanoarrays with a periodicity of (a) $500 \mathrm{~nm}$ and (b) $600 \mathrm{~nm}$. The star (*) shows LSPR positions in (c) with a periodicity of $500 \mathrm{~nm}$ and (d) with a periodicity of $600 \mathrm{~nm}$ 32

Figure 3.6 Experimental refractive index sensitivity for Au nanoarrays with a periodicity of (a) $500 \mathrm{~nm}$ and (b) $600 \mathrm{~nm}$.

Figure 3.7 Simulated UV-Vis spectra of Au nanoarrays with (a) a periodicity of $500 \mathrm{~nm}$, film thickness of $50 \mathrm{~nm}$, and hole diameters changing from $500 \mathrm{~nm}$ to $290 \mathrm{~nm}$ with a step of 30 (b) a periodicity of $600 \mathrm{~nm}$, film thickness of $50 \mathrm{~nm}$, and hole diameters changing from $600 \mathrm{~nm}$ to 390 $\mathrm{nm}$ with a step of $30 \mathrm{~nm}$ 36

Figure 3.8 Simulated UV-Vis spectra of Au nanohole arrays with (a) and (c) a periodicity of 500 $\mathrm{nm}$, hole diameter of $350 \mathrm{~nm}$, and film thickness changing from $10 \mathrm{~nm}$ to $100 \mathrm{~nm}$ with a step of 
$10 \mathrm{~nm}(\mathrm{~b})$ and (d) a periodicity of $600 \mathrm{~nm}$, hole diameter of $420 \mathrm{~nm}$, and film thickness changing from $10 \mathrm{~nm}$ to $100 \mathrm{~nm}$ with a step of $10 \mathrm{~nm}$. 37

Figure 3.9 Simulated UV-Vis spectra of Au nanohole arrays with varying periodicities from 100 to $1000 \mathrm{~nm}$. (a) and (b): the ratio of hole diameter to periodicity is kept constant at 0.7 and the Au film thickness remains at $50 \mathrm{~nm}$; (c) and (d): the hole diameter and Au film thickness remain constant at $350 \mathrm{~nm}$ and $50 \mathrm{~nm}$, respectively. 39

Figure 3.10 (a) Experimentally measured SERS response of Au nanoarrays with a periodicity of $500 \mathrm{~nm}$, a film thickness of $50 \mathrm{~nm}$, and hole diameters changing from $500 \mathrm{~nm}$ to $290 \mathrm{~nm}$ with a step of $30 \mathrm{~nm}$. The Raman signals were acquired under $785 \mathrm{~nm}$ laser from thiophenol (TP) which was coated on Au nanoarrays. Four prominent peaks of TP were plotted in the figure. (b) SEM image of Au nanohole arrays with a periodicity of $500 \mathrm{~nm}$, a film thickness of $50 \mathrm{~nm}$, and a hole diameter of $350 \mathrm{~nm}$. It reveals that experimentally fabricated Au nanohole arrays are not in perfect order. The defects may have an influence on the overall Raman performance. (c) A comparison of simulated electric field enhancement factors of ordered and disordered $\mathrm{Au}$ nanoarrays, simulated reflection spectra, and measured Raman intensity on Au nanoarrays with a periodicity of $500 \mathrm{~nm}$, a film thickness of $50 \mathrm{~nm}$, and hole diameter changing from $500 \mathrm{~nm}$ to $290 \mathrm{~nm}$ with a step of $30 \mathrm{~nm}$. Both the defects and strong reflections of Au nanoarrays were taken into account in understanding the factors that contribute to the overall Raman performance. (d) A comparison of the measured Raman intensity and the calculated Raman intensity. For the calculated Raman intensity, the defects and reflection were linearly combined with perfect order nanoarrays to give an overall contribution to the Raman performance. 
Figure 3.11 (a) Experimentally measured SERS response of Au nanoarrays with a periodicity of $600 \mathrm{~nm}$, a film thickness of $50 \mathrm{~nm}$, and hole diameters changing from $600 \mathrm{~nm}$ to $390 \mathrm{~nm}$ with a step of $30 \mathrm{~nm}$. The Raman signals were acquired under $785 \mathrm{~nm}$ laser from thiophenol (TP) which was coated on Au nanoarrays. Four prominent peaks of TP were plotted in the figure. (b) SEM image of Au nanohole arrays with a periodicity of $600 \mathrm{~nm}$, a film thickness of $50 \mathrm{~nm}$, and a hole diameter of $420 \mathrm{~nm}$. It reveals that experimentally fabricated Au nanohole arrays are not in perfect order. The defects may have an influence on the overall Raman performance. (c) A comparison of the simulated electric field enhancement factors of ordered and disordered $\mathrm{Au}$ nanoarrays, simulated reflection spectra, and measured Raman intensity on Au nanoarrays with a periodicity of $600 \mathrm{~nm}$, a film thickness of $50 \mathrm{~nm}$, and hole diameter changing from $600 \mathrm{~nm}$ to $390 \mathrm{~nm}$ with a step of $30 \mathrm{~nm}$. Both the defects and strong reflections of Au nanoarrays were taken into account in understanding the factors that contribute to the overall Raman performance. (d) A comparison of the measured Raman intensity and calculated Raman intensity. For the calculated Raman intensity, the defects and reflection were linearly combined with perfect order nanoarrays to give an overall contribution to the Raman performance. All the data are normalized......

\section{Chapter Four}

Figure 4.1 (a) SEM image of Au nanostar; (b) UV-Vis absorption spectra of Au nanostar and Au nanostar@MGITC@SiO2; (c) and (d) SEM images of Au nanostar@MGITC@SiO2............... 51 
Figure 4.2 (a) to (g) Protocol for Au nanohole arrays fabrications; (h) SEM image of fabricated Au nanohole arrays with a periodicity of $600 \mathrm{~nm}$, a hole diameter of $420 \mathrm{~nm}$, film thickness of 50

nm. 53

Figure 4.3 SERS sensor working conditions optimization (a) Ionic strength optimization. The optimal ionic strength was found to be $30 \mathrm{mM}$; (b) Incubation time optimization with the optimal value of $16 \mathrm{~min}$; (c) $\mathrm{pH}$ optimization. Neutral $\mathrm{pH}$ is preferred. 55

Figure 4.4 Schematic illustration of the functionalization of Au nanostar@MGITC@SiO2, Au film, and the SERS sensor operation principle. Au nanostar@MGITC@SiO2 sandwich was functionalized with single-stranded DNA sequence of 5'- /5AmMC6/CT CCC CAT A -3'; Au film was functionalized with single-stranded DNA sequence of 5'- /5AmMC6/TA TCC CCA G $3^{\prime}$ 55

Figure 4.5 (a) SERS spectra of Au nanostar@MGITC@ $\mathrm{SiO}_{2}$ on Au nanohole array in the SERS sensor for the detection of $\mathrm{Ag}^{+}$. (b) Plots of SERS peak intensity at $1174 \mathrm{~cm}^{-1}$ as a function of the logarithmic concentration of $\mathrm{Ag}^{+}$. (c) Calibration of the linear range of (b). (d) Performance of the SERS sensor. The black curve stands for the sensor on Au nanohole array while the red curve stands for that on Au film. 58

Figure 4.6 Selectivity test of the SERS sensor against metal ions: $\mathrm{Al}^{3+}, \mathrm{Ca}^{2+}, \mathrm{Cr}^{3+}, \mathrm{Cu}^{2+}, \mathrm{Fe}^{3+}$, $\mathrm{In}^{3+}, \mathrm{In}^{3+}, \mathrm{Ni}^{2+}, \mathrm{Y}^{3+}, \mathrm{Zn}^{2+}$, a mixture of the above ions with $\mathrm{Ag}^{+}$, and $\mathrm{Ag}^{+}$. All of the solution has a concentration of $50 \mathrm{nM}$ in the buffer solution of $10 \mathrm{mM}$ MOPS and $30 \mathrm{mM} \mathrm{NaNO}$ at $\mathrm{pH}$ 7.0. 
Figure 4.7 (a) SEM image of Au nanohole array with a periodicity of $600 \mathrm{~nm}$; the dashed red and green rectangles correspond to (b) and (c) in FDTD simulations. (b) One kind of FDTD simulation cell: point E shows Au nanostar is put at the center of a nanohole (c) The other kind of FDTD simulation cell with Au nanostar put at four different locations: point A is in the middle of the gap between two closest nanoholes; point $\mathrm{B}$ is at the right edge of a nanohole; point $\mathrm{C}$ is in the center of the gap between three closest nanoholes; point $\mathrm{D}$ is at the lower edge of a nanohole.

Figure 4.8 FDTD simulated electromagnetic field distributions with $785 \mathrm{~nm}$ incident light. (a) and (d) show the electromagnetic fields on Au nanohole arrays with a periodicity of $600 \mathrm{~nm}$ and hole diameter of $420 \mathrm{~nm}$ using $\mathrm{x}$ - and y-polarized incident light, respectively; (b) to (c) and (e) to (f) show the field enhancement of Au nanostars located at different places on Au nanohole array with $\mathrm{x}$ - and y-polarized light, respectively. 62

Figure 4.9 FDTD simulated electromagnetic field distributions with a y-polarized $785 \mathrm{~nm}$ incident light. (a) the field distribution on Au nanohole array with a periodicity of $600 \mathrm{~nm}$ and diameter of $420 \mathrm{~nm}$; (b) the field enhancement of Au nanostar at the center of nanohole. 63

Table 4.1 SERS enhancement factors of Au nanostars at different locations on Au nanohole array. The SERS enhancement factors in the table were from the M-N and R-S cross-sections. The MN corss-section was shown in Figure 4.7 (c); R-S cross-section was shown in Figure 4.7 (d).... 63 


\section{List of Equations}

(1) $\epsilon_{d}+\epsilon_{m}=0$

(2) $w_{s p}=\frac{w_{p}}{\sqrt{\epsilon_{d}+1}}$ 7

(3) $k_{s p p}^{2}=\left(\frac{w}{c}\right)^{2} \frac{\epsilon_{d} \epsilon_{m}}{\epsilon_{d}+\epsilon_{m}}$ 8

(4) $k_{s p p}=\frac{w}{c} \sqrt{\epsilon_{d}} \sin \theta$. 10

(5) $k_{s p p}=\frac{w}{c} n_{d} \sin \theta+m \frac{2 \pi}{p} i+n \frac{2 \pi}{p} j$

(6) $w_{l}=w_{p}\left[\frac{l}{\epsilon_{d}(l+1)+l}\right]^{1 / 2}$

( 7 ) $\eta=64(k r)^{4} / 27 \pi^{2}$

(8) $\lambda_{\text {spp }}=a_{o}\left[\frac{4}{3}\left(i^{2}+i j+j^{2}\right)\right]^{-1 / 2} \operatorname{Re}\left(\frac{\epsilon_{d} \epsilon_{m}}{\epsilon_{d}+\epsilon_{m}}\right)^{1 / 2}$

(9) $w_{\text {hole }}^{2}+w_{\text {particle }}^{2}=w_{p}^{2}$

(10) $R=m \Delta \eta=m\left(\eta_{\text {final }}-\eta_{\text {initial }}\right)$

(11) $G_{S E R S}=\left|E_{\text {loc }}\left(w_{\text {exc }}\right) / E_{\text {inc }}\left(w_{\text {exc }}\right)\right|^{4}$ 19 


\section{List of Tables}

Table 4.1 SERS enhancement factors of Au nanostars at different locations on Au nanohole array. The SERS enhancement factors in the table were from the M-N and R-S cross-sections. The M-

N corss-section was shown in Figure 4.7 (c); R-S cross-section was shown in Figure 4.7 (d)... 63

Table 4.2 SERS enhancement factors of Au nanostars at different locations on Au nanohole array.

The SERS enhancement factors in the table were from the X-Y cross-sections........................ 63 


\section{Chapter 1: Introduction}

\subsection{Motivation}

Plasmonics allows the manipulation of light using resonances controllable through nanofabrication. This is especially evidenced in the case of metallic films, which after patterning with nanoscale holes, transform into a tunable optical concentrator with multiple possible modes. ${ }^{1-4}$ While flat metal films can support surface plasmon resonance, the momentum mismatch prevents incident light from exciting this mode. When patterned, the periodic hole array supplies the extra momentum at a resonance wavelength determined by the pitch and hole size, creating unique plasmonic phenomena such as extraordinary optical transmission and local electromagnetic field concentration.

The ability of surface plasmon resonance (SPR) to manipulate light has evolved the phenomenon into a powerful analytical tool ${ }^{5}$. In 1902, Wood first observed rapid intensity variations on narrow spectral regions in his reflection gratings experiment, which is now called Wood's anomalies ${ }^{6}$. While this was the first observation of the phenomenon of SPR, it took quite a few great scientists, such as Lord Rayleigh and Fano, several decades before a complete theory was established. In 1957, Ritchie first theoretically introduced $\mathrm{SPR}^{7}$. In 1968, 66 years after the first observation of SPR by Wood, Otto successfully excited SPR through the evanescent field in an attenuated internal reflection method.

SPR is found in two unique forms, surface plasmon polaritons (SPP) and localized surface

plasmon resonance (LSPR), and this duality is found in gold nanohole arrays. SPP leads to extraordinary optical transmission through the nanoholes, while LSPR leads to an enhanced 
absorption and intense concentration of the local electromagnetic field. The LSPR and SPP shift with changes in the local refractive index, allowing for biomolecular interaction monitoring.

Commercially available SPR sensors are built based on the Kretschmann configuration ${ }^{8}$ or Otto configuration ${ }^{9}$, which involve using a prism to supply the necessary momentum to excite SPR in a thin metal film. These types of sensors are not molecule specific, and require a complicated experimental setup. Hole arrays allow for the removal of the coupling prism, simplifying the sensor. Further, nanohole arrays support both LSPR and SPP, which have different refractive index sensitivities and effective sensing distances. The local electromagnetic field can also be used for SERS, allowing molecule specific detection.

Despite these advantages, the dual optical response of nanohole arrays remains poorly understood, with the complex crossover between localized and propagating modes, as well as the possible field enhancements, preventing the systematic design of SERS based sensors. Therefore, in this thesis, not only will the experimental parameters that control the LSPR and SPP modes be explored, but finite difference time domain (FDTD) analysis will also be used to explore the physical origin of each mode. The knowledge gained is then used to build an optimized SERS sensor for Ag ion detection, resulting in a limit of detection of $170 \mathrm{fM}$.

\subsection{Objectives}

The thesis is dedicated to the investigation and manipulation of the plasmonic field supported by Au nanohole arrays, as well as to the exploitation of the plasmonic field to build a SERS sensor for environmental monitoring. Specifically, the following aspects are to be investigated:

(1) Implement nanosphere lithography (NSL) technique for fabrication of nanohole arrays 
NSL is a commonly used technique in nanofabrication capable of producing wellcontrolled and well-ordered nanoparticle/nanohole arrays ${ }^{10-12}$. It is characterized by its simple, low-cost, high-throughput, inherently parallel, and materials-general merits. Polystyrene (PS) spheres are used as the pattern mask with deposited metal forming the underlying nanoarray. Different PS sphere sizes and etching of the spheres can result in metallic nanoarrays of different sizes. The correlation of the various etching time of PS spheres with the resulting metallic nanoarrays will be investigated.

(2) Study the evolution of the optical properties of the transition from Au nanotriangle arrays to Au nanohole arrays

In NSL, by controlling the PS spheres etching time, a series of shapes ranging from $\mathrm{Au}$ nanotriangle arrays to Au nanohole arrays can be obtained. This provides the basis for an experimental study of how the optical properties evolve with the transition from localized Au nanotriangles to Au nanohole arrays. FDTD simulations will be used to understand the origin of the measured optical properties.

(3) Investigate the correlation of the optical properties with the SERS performance of Au nanohole arrays

$\mathrm{Au}$ nanohole arrays support strong EM fields which can produce a large SERS enhancement. However, there is a lack of understanding of how the hole geometry, size, pitch, and transition from nanotriangle to nanohole contribute to the overall observed Raman performance. Therefore, the correlation of the optical properties of the Au nanohole arrays with SERS performance will be investigated and explained using FDTD simulations. 
(4) Develop a SERS sensor for silver ions detection based on coupled Au nanostars and nanohole arrays

The study of the optical properties and the correlation with SERS performance will provide a guideline in selecting the appropriate $\mathrm{Au}$ nanohole arrays for optimal performance and how these parameters can be optimized to overcome current drawbacks in Au nanohole arrays based sensors. Further, Au nanostars have demonstrated excellent SERS performance ${ }^{13-16}$, and they will be combined with the Au nanohole array to further improve the detection of silver ions.

\subsection{Significance}

Study of the optical properties of the Au nanoarrays provides us with guidelines needed to control the plasmonic behavior, as well as to uncover the optimal conditions under which the Au nanohole arrays can enhance Raman scattering and allow the development of a SERS sensor. This work manifests its significance in the following aspects:

(1) Exploration of a general trend of size- and shape-dependent resonance modes on $\mathrm{Au}$ nanohole arrays provides a useful guideline in manipulating the electromagnetic fields of nanostructures.

(2) Exploration of the correlation between plasmonic substrates and the optimal SERS performance provides a knowledge that is useful in engineering SERS substrate specific to the needs. 
(3) Exploration of highly sensitive and selective sensors provides a powerful and practical technique on environmental monitoring, biological detection, and early diagnosis of diseases.

\subsection{Thesis organization}

The thesis consists of five chapters. Chapter One gives a general introduction to the topic of the thesis. Chapter Two focuses on the background introduction and literature review of the topic. Chapter Three launches a systematic study of the transition from gold nanotriangle arrays to gold nanohole arrays. A combination of experimental results and FDTD simulations allows an indepth understanding of the optical properties of gold nanohole arrays. In Chapter Four, a SERS sensor is built for silver ions detection using the knowledge learned about hole arrays. Chapter Five is a summary of the results and a brief perspective for future research. 


\section{Chapter 2 Background and Literature Review}

\subsection{Plasmonics}

\subsubsection{Surface Plasmon}

A plasmon is defined as a quasiparticle resulting from the quantization of collective electron oscillations in solids ${ }^{17}$. The plasma frequency of a metal is given by $\omega_{p}=\sqrt{\frac{n_{e}^{2}}{m_{e f f} \epsilon_{0}}}$ where $n_{e}$ is the density of electrons and $m_{\text {eff }}$ is the effective mass ${ }^{18}$.

At the plasma frequency the electrons in the metal oscillate 180 degrees out of phase with the incident field, leading to a negative real part of the dielectric constant and reflection of incident light at frequencies smaller than the plasma frequency. This effect, in combination with interband transitions, gives metals their characteristic color. The high electron density of metals usually places the plasma frequency in the UV to visible range, but heavily doped semiconductors can have near infrared plasma frequencies.

\subsubsection{Surface Plasmon Polaritons}

At frequencies below the plasma frequency the electromagnetic field is stored in the collective electron oscillations, raising the possibility for confining the incident field to sub-wavelength volumes. One way to achieve this is in a thin metal film. The resulting boundary conditions quantize the plasma oscillations, creating surface plasmon polaritons (SPP).

A SPP describes the collective electron oscillations at a dielectric-metallic interface. The SPP behaves like a surface wave and can be obtained by solving Laplace's equation since the net surface charges are zero. Across the interface, both the tangential component of the electric field 
and the normal component of the electric displacement are held continuous in order to satisfy the boundary conditions, which are subject to the condition:

$$
\epsilon_{d}+\epsilon_{m}=\mathbf{0}
$$

where $\boldsymbol{\epsilon}_{\boldsymbol{d}}$ is the dielectric constant of the adjacent dielectric, $\boldsymbol{\epsilon}_{\boldsymbol{m}}$ is the metal dielectric function. The above equation suggests that when the metal and dielectric have opposite sign but equal real parts of the permittivity, a resonance will occur. As discussed, this condition is met at the plasma frequency of the metal ${ }^{19}$.

The frequency of surface plasmon on planer metallic film is determined from the plasma frequency of the metal plus the boundary conditions set by the planar geometry ${ }^{20}$ :

$$
w_{s p}=\frac{w_{p}}{\sqrt{\epsilon_{d}+1}}
$$

where $\boldsymbol{w}_{\boldsymbol{p}}$ is the plasma frequency. In vacuum, the SPP frequency is $\boldsymbol{w}_{\boldsymbol{s} \boldsymbol{p}}=\frac{\boldsymbol{w}_{\boldsymbol{p}}}{\sqrt{2}}$, the surface boundary conditions red-shifting the UV plasma frequency to the visible SPP resonance.

As shown in Figure 2.1, SPP behaves as a planar propagating electromagnetic wave highly confined to the interface and decaying with increasing distance away from the interface. The propagation distance depends on the imaginary part of the dielectric function of the metal. While propagating, it gradually loses energy due to metallic absorption (Ohmic losses) and other radiative and non-radiative scattering ${ }^{21}$. The evanescent SPP field decays exponentially into the metal and the dielectric. The decay length is dielectric-dependent with larger dielectric constants leading to shorter decay lengths. The SPP is tightly confined to the interface, allowing subwavelength concentration of the incident electromagnetic field with strong intensity ${ }^{\mathbf{2 0}}$. 


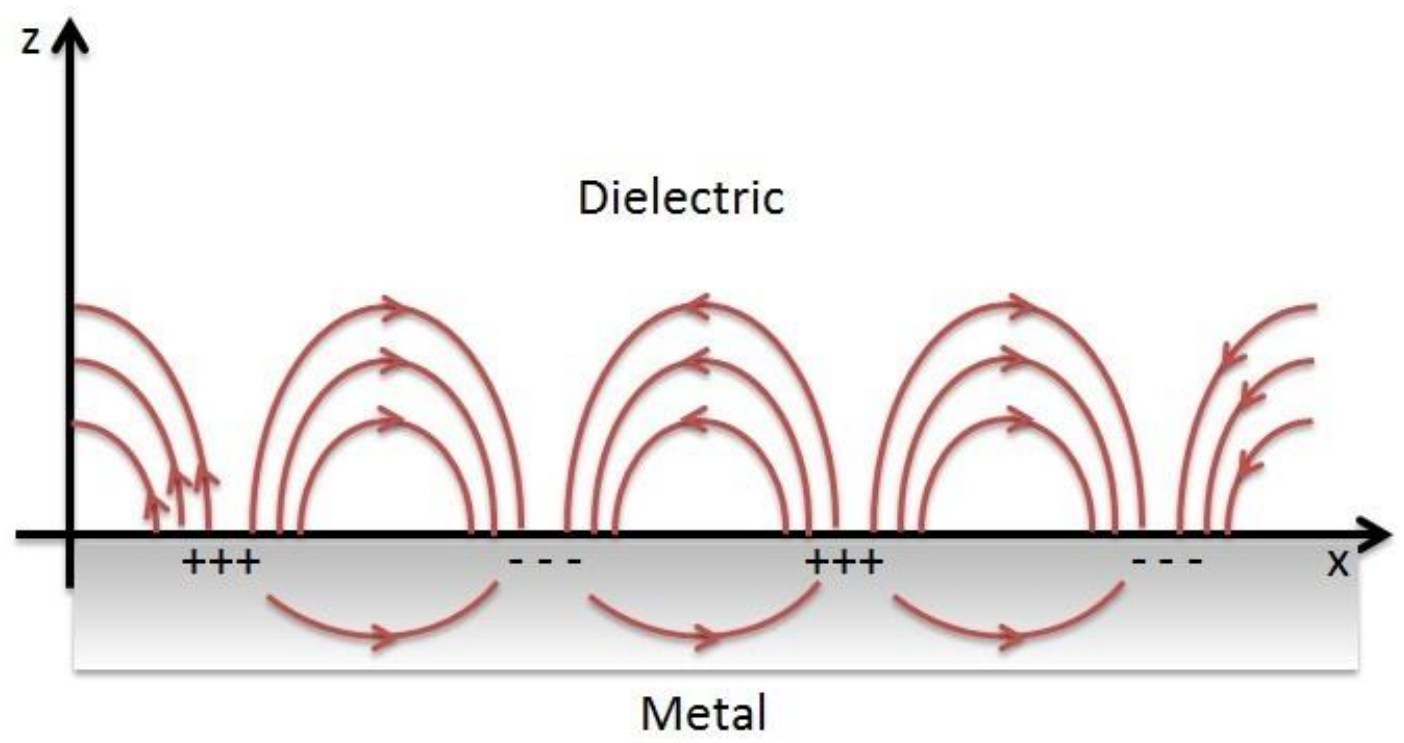

Figure 2.1 Schematic illustration of the surface plasmon polaritions at the interface between the dielectric and the metallic surface ${ }^{22}$.

The SPP can not be excited by incident light due to the momentum mismatch between the SPP sub-wavelength wavevector and free space. The momentum of the SPP on a smooth metallic surface can be derived from the dispersion relation ${ }^{20}$ :

$$
k_{s p p}^{2}=\left(\frac{w}{c}\right)^{2} \frac{\epsilon_{d} \epsilon_{m}}{\epsilon_{d}+\epsilon_{m}}
$$

where $\frac{w}{c}$ is the free space wavevector. For short SPP wavevectors (photon-like excitations), the transverse component of the electric field of SPP dominates, making the dispersion relation curve close to the light line, as shown in Figure 2.2. For long wavevectors (plasmon-like excitations), the transverse and longitudinal components are comparable.

Two independent SPP resonance modes for each dielectric interface can be excited simultaneously for a thick metallic film. The modes are degenerated if the two interfaces are identical. The two independently excited SPP resonance modes will interact provided the 
metallic film is thin enough. The interaction, if strong enough, will significantly modify the dispersion relation and even break the degeneracy of the resonance modes, splitting the surface plasmon frequency into two branches corresponding to symmetric (low frequency mode) and antisymmetric (high frequency mode) electromagnetic field distributions.

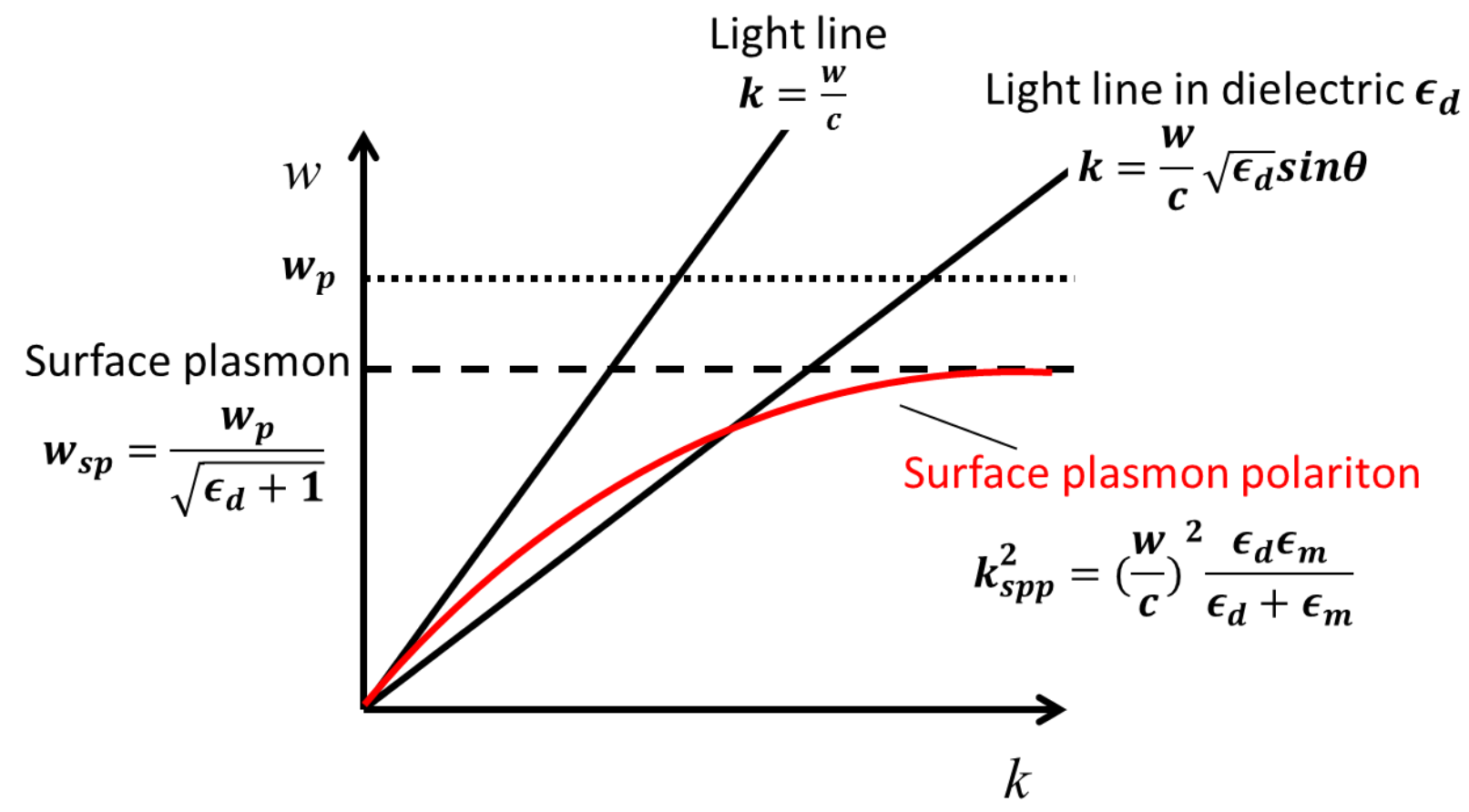

Figure 2.2 Shown in red is the dispersion relation of surface plasmon polaritons on the dielectric-metallic interface. Also shown are the surface plasmon frequency, bulk plasma frequency, the light line in air, and the light line in dielectric.

As the dispersion curve of SPP lies on the larger-wavevector side of the light line ${ }^{20}$, it cannot be excited directly by incident light from the adjacent dielectric (Figure 2.2). In order to excite the SPP, extra momentum must be supplied to balance the SPP wavevector. One popular method is using the evanescent field in total internal reflection or a diffraction grating. Total internal reflection occurs in a prism with a large dielectric constant when the incident angle exceeds the 
critical angle. The incident light gains extra momentum due to the large dielectric constant of the prism and can therefore match the SPP at certain incident angles ${ }^{17}$ :

$$
k_{\text {spp }}=\frac{w}{c} \sqrt{\epsilon_{d}} \sin \theta
$$

Using the evanescent field in total internal reflection is adopted in both Kretschmann and Otto configurations, which are two characteristic concepts in exciting SPP resonance mode ${ }^{9,23,24}$.

Using a diffraction grating is another way to satisfy wavevector conservation. Bragg-like scattering is controlled by the incident angle, periodicity, lattice vector, and dielectric constant. The diffraction coupling condition for a SPP is given by ${ }^{17}$ :

$$
k_{s p p}=\frac{w}{c} n_{d} \sin \theta+m \frac{2 \pi}{p} i+n \frac{2 \pi}{p} j
$$

where $\boldsymbol{n}_{\boldsymbol{d}}$ is the refractive index of the dielectric medium, $m$ and $n$ are integers which determine the propagation direction, $p$ is the periodicity, $i$ and $j$ are the unit lattice vectors.

In summary, SPP behave as a propagating electromagnetic wave highly concentrated at the interface with frequency given by the dispersion relation. SPP cannot be directly excited by incident light, but instead requires additional momentum.

\subsubsection{Localized Surface Plasmon Resonance}

If the thin metal film is further reduced in volume so that the dimensions are smaller than the incident wavelength, the retardation effects are negligible, and the electrons across the nanoparticle oscillate in phase. This phenomenon is known as localized surface plasmon resonance (LSPR) and is usually excited on curved metallic objects, such as metallic 
nanoparticles and voids of various topologies, with the topology supplying the additional momentum to couple directly to incident $\operatorname{light}^{\mathbf{2 0}}$. The electromagnetic properties of LSPR can be approximated electrostatically. The boundary conditions that determine the LSPR frequency are dependent on the shape, size, and dielectric constant of the metallic objects ${ }^{25}$. LSPR allows the incident light to be highly concentrated, leading to an intense local electromagnetic field.

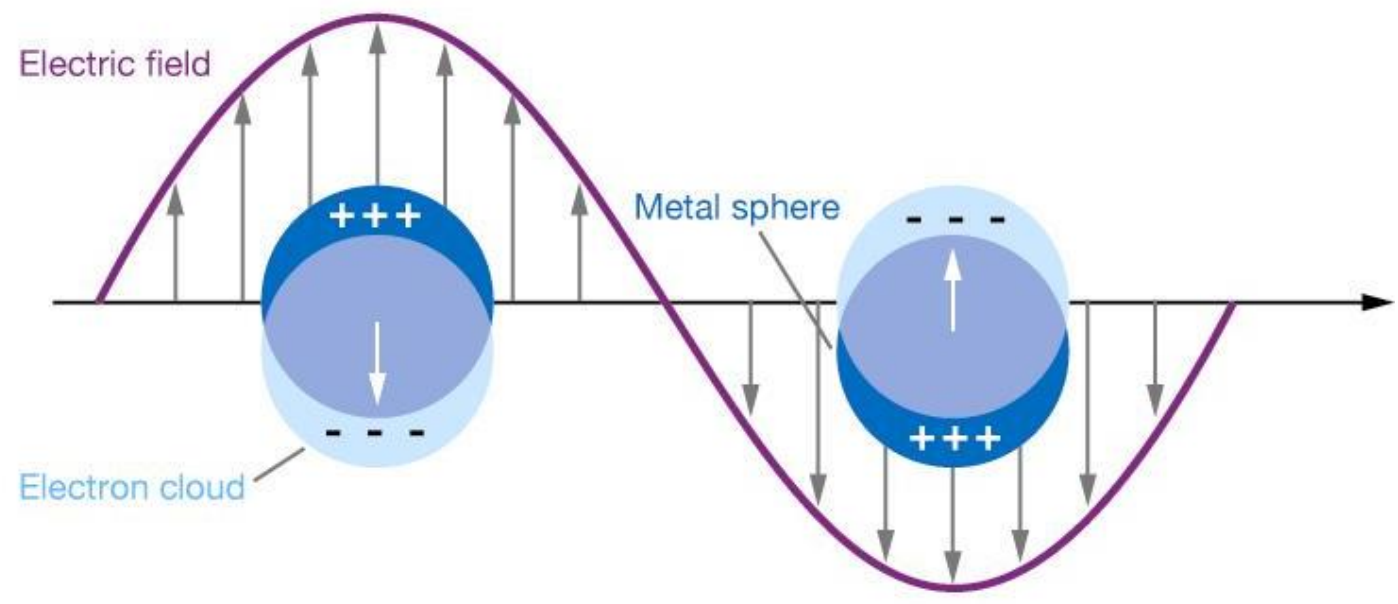

Figure 2.3 Schematic illustration of the dipolar excitation of the localized surface plasmon resonance (LSPR) of metallic nanoparticle ${ }^{26}$.

For a metallic nanopshere whose size is far smaller than the incident wavelength, as shown in Figure 2.3, the frequency of LSPR is given by ${ }^{17}$ :

$$
w_{l}=w_{p}\left[\frac{l}{\epsilon_{d}(l+1)+l}\right]^{1 / 2}
$$

where $l$ is angular momentum of LSPR, $l$ can only be positive integers. The above equation indicates the frequency of the LSPR is discrete, and does not follow a dispersion relation like the SPP. This reflects the fact that LSPR can be excited by free space light without additional momentum matching, with the resonance frequency instead depending on the size of the nanoparticle. For smaller nanoparticles, dipolar resonances are excited for $l=1$. With an increase 
of the nanoparticle size, scattering becomes increasingly dominant and higher resonance modes (like quadruple, octupole, etc.) are excited. In the large size limit, the LSPR resonance approaches that of the surface plasmon resonance on flat metallic surface, i.e. if $\boldsymbol{l} \rightarrow \infty$, then $w_{l} \rightarrow \frac{w_{p}}{\sqrt{\epsilon_{d}+1}}$.

On roughened or defect-containing flat metallic surface, LSPR can occur and excite SPP modes nonradiatively. Conversely, when SPP encounter roughened features or defects, LSPR can be excited $^{\mathbf{2 0}}$. When the frequency of both LSPR and SPP are very close, they couple with each other and lead to a significant enhancement of the electromagnetic field ${ }^{27}$. LSPR-LSPR coupling also happens when the distance among individual nanoparticles are within the decay length. Both forms of plasmonic hybridization lead to even larger field confinement and local enhancement.

\subsection{Optical Properties of Gold Nanohole Arrays}

\subsubsection{Bethe's Theory of Diffraction}

Light interacting with a small pinhole was historically described as traveling through it and creating an inverted image on the other side. A higher resolution image can result from a smaller pinhole. However, this is balanced with diffraction effects which blur the image.

In the $17^{\text {th }}$ century, the phenomenon of diffraction from a circular aperture was first approximated by Grimaldi. Yet, it was not until in 1942 that Bethe ${ }^{28}$ theoretically treated the interaction between electromagnetic radiation and a subwavelength hole exactly. His prediction of a very weak transmission below the cut-off condition dominated the understanding of subwavelength optical properties for over 50 years, until the discovery of extraordinary optical transmission (EOT) in 1998 by Ebbesen ${ }^{1}$. 
Bethe conceived an idealized scenario in which the diffraction of electromagnetic waves took place at a circular hole in a perfectly thin conducting plane. For an incident wavelength $\lambda$, the optical transmission efficiency $\eta$ (normalized to the hole area), follows the relation.

$$
\eta=64(k r)^{4} / 27 \pi^{2}
$$

where $\mathbf{k}=\mathbf{2} \boldsymbol{\pi} / \boldsymbol{\lambda}$ describes the incident wavevector and $\mathbf{r}$ is the radius of the hole. From equation (1), the optical transmission efficiency decreases as the fourth power of the ratio of the

aperture radius to the incident wavelength, i.e. $\eta \propto\left(\frac{r}{\lambda}\right)^{4}$. This relationship has been found to hold in many materials except those that support surface plasmon resonances.

\subsubsection{Extraordinary Optical Transmission}

Extraordinary optical transmission, or EOT ${ }^{1-3,29-34}$, is the anomalous increase in the optical transmission through subwavelength apertures in metallic film during the excitation of surface plasmon polaritons $(\mathrm{SPP})^{4,33,35}$. It was first discovered by Ebbesen in 1998. ${ }^{1}$ EOT occurs because SPP, while propagating along the interface of the metal and dielectric, can also tunnel through the holes which act as a waveguide. The hole array acts as a diffraction grating, giving the extra momentum needed to couple incident light to the SPP mode. Therefore, when the excited SPP encounters the subwavelength apertures while propagating on the metallic film, it can radiatively scatter back into the far field. If the SPP first tunnels through the sub-wavelength apertures or couples to the other side of the film, the light will be re-radiated on the opposite side from the incident light, creating a higher transmission than predicted by Bethe. 


\subsubsection{Optical Properties of Gold Nanohole Arrays}

Hole arrays are unique plasmonic substrates in that they can support both SPP and LSPR modes simultaneously. SPP is involved in the enhanced transmission as discussed ${ }^{21,36}$. The position of SPP resonance can be estimated according to the SPP dispersion relation and the diffraction coupling condition described in section 2.1.2. For hexagonally patterned nanohole arrays, as created by NSL, the SPP resonance is given by ${ }^{17}$ :

$$
\lambda_{\text {spp }}=a_{o}\left[\frac{4}{3}\left(i^{2}+i j+j^{2}\right)\right]^{-1 / 2} \operatorname{Re}\left(\frac{\epsilon_{d} \epsilon_{m}}{\epsilon_{d}+\epsilon_{m}}\right)^{1 / 2}
$$

where $a_{o}$ is the lattice constant of the periodic nanostructure; $i$ and $j$ are the lattice indices; $\epsilon_{d}$ and $\epsilon_{m}$ represent the dielectric constant of the dielectric and metallic film, respectively.

Opposite of the propagating SPP mode, the LSPR mode is confined to the edge area of the nanoholes. The LSPR frequency of a nanohole is complementary to that of a nanoparticle of the same size and shape, subject to the condition: ${ }^{20}$

$$
w_{\text {hole }}^{2}+w_{\text {particle }}^{2}=w_{p}^{2}
$$

where $w_{\text {hole }}$ is the LSPR frequency of a nanohole, $w_{\text {particle }}$ is the LSPR frequency of the nanoparticle that has the same size and shape as the nanohole, $w_{p}$ is the bulk plasma frequency of the metal. The LSPR resonance leads to an increase in absorption or reflection in the UV-Vis spectrum.

Another phenomenon that is often observed in nanohole arrays is Wood's anomaly ${ }^{37}$. Wood's anomaly takes place at diffraction gratings when a diffracted order becomes tangent to the plane of grating. It also appears as a transmission dip, however, with defining different origins and 
characteristics compared to LSPR. As LSPR behaves as a kind of standing electromagnetic wave, its feature has nothing to do with the incident angle. In contrast, Wood's anomaly is subject to a red shift with an increase of the incident angle due to the diffraction gratings ${ }^{38}$.

The above description of resonance modes suggest the optical properties of Au nanohole arrays are dominated by SPP and LSPR ${ }^{39-44}$. Thus, control of both resonance modes and their coupling will determine the optical performance of the Au nanohole arrays. The LSPR of Au nanohole arrays can be manipulated by controlling the incident radiation, the hole shape and diameter, periodicity, metal composition, and dielectric environment ${ }^{45-50}$. In Au nanohole arrays, SPP are excited when the diffraction grating conditions matches the SPP wavevector, ${ }^{51}$ determined by the periodicity $^{52}$ and incident angle. Cubic ${ }^{46,53,54}$ and hexagonal ${ }^{41,55}$ patterned periodic nanohole arrays differ in the direction in which the lattice wavevector is balanced against the SPP wavevetor as well as the angle-resolved resonant excitation conditions. The highly symmetric hexagonal nanohole arrays are less affected by changes in the incident angle than the cubic nanohole arrays.

\subsection{Sensing with Au Nanohole Arrays}

\subsubsection{Refractive Index Based SPR Sensors}

The SPR peak shifts when the local refractive index is changed, such as due to the adsorption of analytes $^{36,56}$, forming the basic principle of colorimetric SPR biosensors. This type of sensor is demonstrated in Figure 2.4, where a Au nanohole array chip is first functionalized with ligands. Next, analytes in solution are captured by the ligands-modified Au nanohole array, changing the local refractive index and shifting the SPR peak. This type of sensor is possible using both SPP and LSPR modes. 


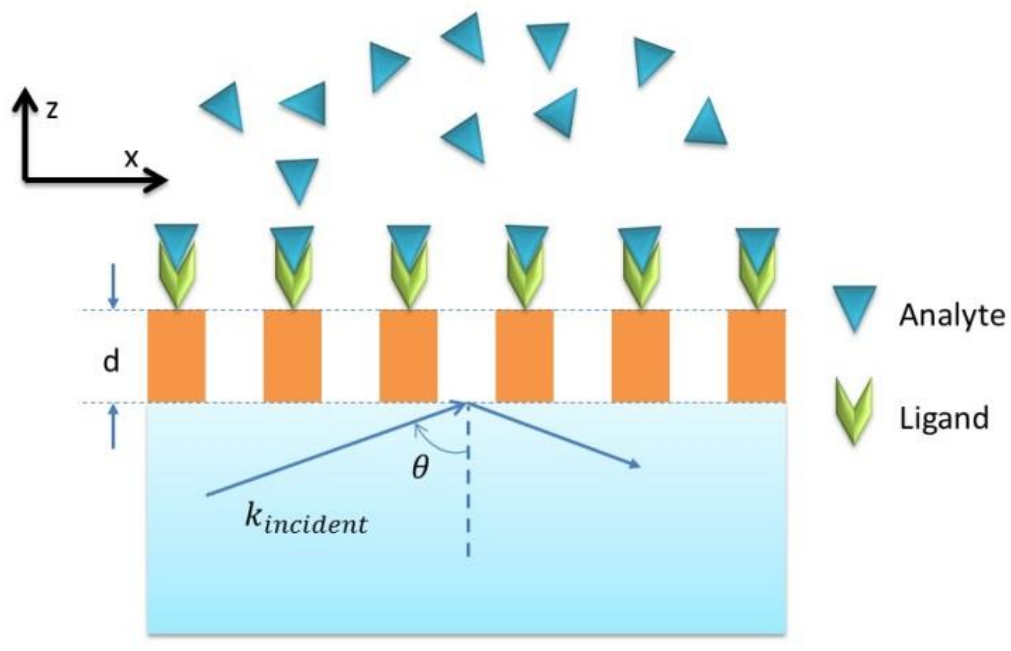

Figure 2.4 Scheme of mechanism of SPR Sensor: ligand capturing the analyte on nanohole arrays, shifting the SPR peak.



Figure 2.5 The sensorgram shows a cycle of SPR sensing process

The dynamics of the capture process can be monitored by the shift in the SPR peak as shown in Figure 2.5. There are three stages. First, the association stage (point A to point B, with dissociation neglected): accumulation of analytes over time continuously shifts the SPR peak; 
second, dissociation stage (C to D): the dissociation of captured analytes shifts the SPR peak back; third, regeneration stage (E to F): removal of all the captured analytes for repeated use.

For most SPR sensors, the SPR peak shift is linearly related to the refractive index change, which is given by ${ }^{57}$ :

$$
\boldsymbol{R}=\boldsymbol{m} \Delta \boldsymbol{\eta}=\boldsymbol{m}\left(\boldsymbol{\eta}_{\text {final }}-\boldsymbol{\eta}_{\text {initial }}\right)
$$

where $\mathrm{R}$ is the SPR peak shift, and $m$ stands for the sensitivity of the SPR sensor with a unit of RIU. Refractive index before and after analytes are captured is denoted by $\eta_{\text {initial }}$ and $\eta_{\text {final }}$, respectively.

Originally, most SPR based refractive index sensors relied on the Otto or Kretschmann configuration. Au nanohole array based SPR sensors, however, can take advantage of extraordinary optical transmission and therefore are often used in transmission mode under normal incidence ${ }^{58}$. The sensor can be applied to monitor biological reactions or molecular bonding merely by immersing the Au nanohole array chip into the solution ${ }^{58,59}$. This greatly reduces the complexity of the optical setup ${ }^{60,61}$, especially compared to angle resolved SPR sensors which rely on coupling prisms. LSPR can be used similarly, using free space light to measure the amount of analyte present, reducing experimental complexity. The rapid development of fabrication techniques in the past decade has further reduced the complexity of SPR based refractive index sensors by integrating the plasmonic substrate or nanoparticle into a microfluidic chip ${ }^{62-64}$. The microfluidic chip is composed of a glass substrate which supports the $\mathrm{Au}$ nanohole array and channels which facilitate solution flow, miniaturizing the entire experimental setup. 
The first report of microfluidics integrated nanohole arrays ${ }^{65}$ used twelve square nanohole arrays that had been fabricated and integrated into a microfluidic channel for monitoring the biochemical bonding process in the biotin-streptavidin system with a sensitivity of $333 \mathrm{~nm} / \mathrm{RIU}$. Similar work ${ }^{66-74}$ has been reported for real-time, label-free multiplex SPR sensing with analytes placed on the surface of metallic nanohole arrays. SPR devices have further been built and integrated with microscopes as well as portable fiber-optic spectrometers, greatly simplifying the detecting process and allowing point of care application ${ }^{75,76}$.

Apart from flow-over SPR sensors, flow-through SPR sensors were also developed taking advantage of the rapid cross-stream transport through the nanoholes. For flow-through SPR sensors $^{77-79}$, the holes are open on both sides, making the reactants free to transport through the nanoholes and thus increasing the chance for analytes to be captured ${ }^{77-79}$. Flow-through SPR sensors are limited to the detection of small proteins.

In summary, refractive index based Au nanohole array sensors possess the advantages of a simple configuration and straightforward operating principle, but suffer from low sensitivity and are incapable of molecular identification.

\subsubsection{SERS Sensors}

The deficits of refractive index based sensors can be overcome using surface enhanced Raman scattering (SERS). As a non-invasive optical technique capable of measuring molecular fingerprints, surface-enhanced Raman spectroscopy ${ }^{80}$ has long been applied in biomedical

diagnosis, environmental monitoring, and even homeland security ${ }^{81-83}$. SERS is primarily based on the local concentration of the electromagnetic field in plasmonics increasing the small Raman scattering cross section ${ }^{84}$. 
There are two major mechanisms, however, that can be responsible for the SERS enhancement: EM enhancement ${ }^{84,85}$ and chemical enhancement $(\mathrm{CE})^{86}$. While the EM enhancement is attributed to the coupling between the incident radiation and surface plasmon resonances, the $\mathrm{CE}$ can be ascribed to charge transfer between the metallic nanoparticle and the molecule. Both mechanisms often co-exist in most SERS systems, with the EM field enhancement usually being orders of magnitude larger than the CE enhancement. This is because the local EM field undergoes incident enhancement and emitted enhancement, giving the overall SERS enhancement scaling to the fourth power with the local EM field ${ }^{87,88}$ :

$$
G_{S E R S}=\left|E_{\text {loc }}\left(w_{\text {exc }}\right) / E_{\text {inc }}\left(w_{\text {exc }}\right)\right|^{4}
$$

where $E_{l o c}\left(w_{\text {exc }}\right)$ and $E_{i n c}\left(w_{\text {exc }}\right)$ are the amplitudes of the local and incident electric fields at an excitation frequency of $w_{\text {exc }}$. The chemical enhancement on the other hand makes a relatively small contribution to SERS because it happens when charge transfers from the metal to molecule or vice versa under excitation, providing only one enhancement step which is highly dependent on the local energy alignment. A typical CE process gives an enhancement of $\sim 10^{2}$.

$\mathrm{Au}$ nanohole arrays are promising for enhancing SERS because of the local EM field concentration due to the LSPR and SPP modes. The enhanced transmission in Au nanohole arrays was found to enhance SERS as a result of SPP involvement ${ }^{89-92}$. Under the enhanced transmission condition, SPP were excited concentrating the EM field at the interface. As long as the Raman laser excitation wavelength overlapped with the enhanced transmission peak, the SERS signal was boosted due to the strong planer EM field. The LSPR of the Au nanohole array was also used for a SERS enhancement, with the enhancement originating in the confined local electromagnetic field, just like in Au nanoparticles ${ }^{93}$. Raman molecules must be placed near the 
edge area of the hole array in order to benefit from the local EM field, since it is concentrated in this region.

To better understand the SERS enhancement from Au nanohole arrays, many studies have been devoted to control the parameters of $\mathrm{Au}$ nanohole arrays and measure the resulting optical properties. It was initially found that a narrower metallic film gap spacing led to higher SERS enhancement factors $^{93-96}$, given that the narrower gap better concentrated the electromagnetic field. However, a later study on nanotriangle and nanohole arrays revealed that there was a strong correlation between the SERS performance and the ratio of the hole diameter to the periodicity of nanoarrays ${ }^{97}$. For a ratio of one, the hot spots were located at the tips of nanotriangle arrays. When the ratio reduced to $0.75 \sim 0.7$, nanohole arrays were created and optimal SERS performance was consistently observed in this region. It was also observed the hot spots moved to the center of the nanoholes when the ratio reduced to less than 0.4 . The origin of the SERS enhancement and optimal configuration, as well as the mixing between the SPP and LSPR modes, remains unclear.

In summary, the advantages and challenges for using Au nanohole arrays to build a SERS sensor co-exist.

First, the electric field is delocalized over the surface of gold nanohole arrays under the SPP resonance and localized in a periodic fashion for the $\operatorname{LSPR}^{97}$, leading to a homogeneously distributed field which can provide a highly repeatable and robust Raman signal. Second, gold nanohole arrays support multiple resonance modes covering a wide range of the UV-Vis-NIR spectrum, allowing flexible selection of the excitation wavelength. Third, the fact that gold nanohole arrays are often fabricated on glass or quartz slides makes the integration of a SERS 
sensor device into microfluidics easy. Yet, there is an ambiguous understanding of how the resonance modes are correlated to the optimal SERS performance, particularly as to whether the LSPR or SPP is allowing the enhancement. SPP and LSPR are likely to co-exist on Au nanohole arrays under most circumstances, yet without understanding the origin of each mode, the overall optical response can not be optimized for use in a SERS based sensor. Further, the hole array is a planar substrate, and given the analytes exist in the full volume above the chip, it is wished to further extend the field in a three-dimensional plasmonic substrate. 


\section{Chapter 3: Optical Studies of the Transition from Gold Nanotriangle Arrays to Gold Nanohole Arrays}

\subsection{Introduction}

The phenomenon of extraordinary optical transmission (EOT) ${ }^{1}$ is attributed to light-matter interactions mediated by surface plasmon polaritons (SPP) in subwavelength nanohole arrays ${ }^{98}$. In a patterned metal film the hole array allows the extra momentum needed to directly excite a SPP. Further, the SPP on the incident light side can couple or transfer to the opposite side of the metal film through the nanohole array. The SPP can then re-radiate into the far field again because of the additional momentum supplied by the hole array. This allows for a higher transmission of light than otherwise possible through the metal nanohole, since the subwavelength SPP mode couples through the metal film and then re-radiates.

In recent years, SPP have been intensively studied in nanohole arrays because of the availability of several fabrication methodologies, such as e-beam lithography (EBL), focused ion beam milling (FIB), nanoimprint, and nanosphere lithography (NSL), which provide a high degree of control over size and shape and are capable of producing nearly defect-free nanohole arrays on a large scale. Of the aforementioned techniques, NSL offers a cheap and facile route for parallel manufacture of nanostructures ranging from nanotriangle arrays to nanohole arrays. The different shapes are accomplished by applying oxygen plasma etching to the polystyrene sphere pattern prior to metal deposition. While no etching leads to a nanotriangle arrays since the metal can only fill the holes between the polystyrene spheres, etching with longer times decreases the sphere radius and increases the open area between spheres, resulting in nanohole arrays. 
Nanotriangle arrays are also known as Fischer's patterns ${ }^{99}$, and are dominated by the localized surface plasmon resonance (LSPR) response. The LSPR can be tuned by changing the materials, edge size, and gap of the distance between adjacent nanotriangles ${ }^{99}$. For a single metallic nanotriangle, the electromagnetic field is concentrated around the sharp tips. For the pair of facing nanotriangles which make up a bowtie pair, the electromagnetic fields is confined in the gap when the input polarization aligns with the bowtie nanotriangles interparticle axis ${ }^{100}$. The large electromagnetic field has led to bowtie nanotriangles being used frequently in applications ${ }^{15}$ such as surface enhanced Raman scattering (SERS) detection of biological substances.

When oxygen plasma etching is applied, the PS spheres shrink, and more material is added to the nanotriangle array until a nanohole array results. The periodicity of the subwavelength nanohole array provides additional momentum allowing free space excitation of surface plasmon polaritons (SPP). Multiple orders of the SPP resonances exist. The wavelengths of the various SPP modes are highly dependent on the periodicity, as well as the dielectric environment in proximity to the surface of the metallic nanostructure.

In addition to the SPP, LSPR can also co-exist in the nanohole array with the near field focused at the rim of the nanohole ${ }^{101}$. It is expected that a complex interplay will exist as the plasmon evolves from the LSPR mode in the nanotriangles to the periodic/localized mode in the nanohole array. For an array of nearly connected nanotriangles the field should be focused in the narrow gap, similar to a bowtie array ${ }^{99}$. As the nanotriangles form into holes, the field changes from being focused in the gap to around the rim of the nanohole. This local field can exist at the same time as the SPP travelling charge waves, and the intersection could lead to interesting optical properties. 
Therefore, in this Chapter the optical properties of the evolution of an Au nanotriangle array into an Au nanohole array are systematically investigated.

\subsection{Experimental section}

\subsubsection{Materials}

Quartz slides (AdValue Technology), polystyrene microspheres, glycerine, thiophenol (TP) (Alfa Aesar), Deionized (D.I.) water produced by the Milli-Q Integral 3/5/10/15 system $(18.2 \mathrm{M} \Omega \cdot \mathrm{cm}$, Millipore Corp., USA)

\subsubsection{Instruments}

Au nanotriangle arrays and Au nanohole arrays were characterized under a JEOL JSM-7600F scanning electron microscope (SEM). Ocean Optics USB 4000 spectrometer was used to acquire the transmission and reflection spectra of fabricated Au nanotriangle arrays and Au nanohole arrays. Raman spectra were acquired with an Inspector Series, DeltaNu spectrometer.

\subsubsection{Preparation of Au nanoarrays}

The protocol for Au nanohole arrays fabrication using nanosphere lithography is shown in Figure 3.1. Quartz slides were cut into small pieces (around $1 \mathrm{~cm} \times 1.5 \mathrm{~cm}$ ). They were then washed by immersing into piranha base $\left(\mathrm{H}_{2} \mathrm{SO}_{4}: \mathrm{H}_{2} \mathrm{O}_{2}=3: 1\right.$ at $\left.90{ }^{\circ} \mathrm{C}\right)$ for two hours. The quartz slides were next successively sonicated in acetone, methanol, and D.I. water for $15 \mathrm{~min}$, respectively. After that, polystyrene (PS) microspheres were drop-coated on the cleaned quartz slides to form a monolayer PS sphere template. In our study, PS microspheres with two different diameters $(500 \mathrm{~nm}$ and $600 \mathrm{~nm}$ ) were used. After natural drying, the coated quartz slides were put under oxygen plasma etching for various durations (from 0 min to 10.5 min with a step of 1.5 min). E-beam evaporation was first used to deposit a layer of $\mathrm{Ti}$ ( $5 \mathrm{~nm}$ in thickness) to enhance 
the adhesion between quartz substrate and Au nanohole array, followed by a $45 \mathrm{~nm}$ Au layer. With a total thickness of $50 \mathrm{~nm}$ Ti-Au deposited, the PS microspheres were removed by sonication in methanol. The as-prepared Au nanoarrays were finally rinsed with D.I. water and dried by compressed nitrogen gas.

Figure 3.2 and Figure 3.3 show SEM images of the Au nanoarrays with various hole diameters. Au nanotriangle arrays were formed if no etching was used. For short etching times, the individual triangle nanoparticles became partially connected, forming bow-tie nanostructures. After 4.5 min of etching, the Au nanohole array pattern showed up as a very thin bridge that connects each triangle nanotriangle, completing the transition from an individual Au nanotriangle array. For Au nanohole arrays with a periodicity of $500 \mathrm{~nm}$ under $4.5 \mathrm{~min}$ etching, the hole diameter was reduced to around $410 \mathrm{~nm}$; for Au nanohole arrays with a periodicity of $600 \mathrm{~nm}$ under 4.5 min etching, the hole diameter was reduced to around $510 \mathrm{~nm}$.

(a) Substrate Pre-cleaning



(b) Microsphere coating

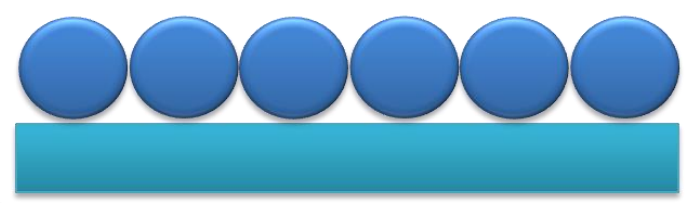

(c) Microsphere Etching

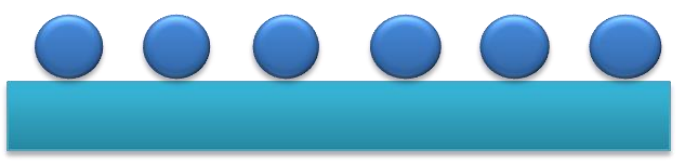

(d) Ti Layer Deposition



(e) Au Layer Deposition

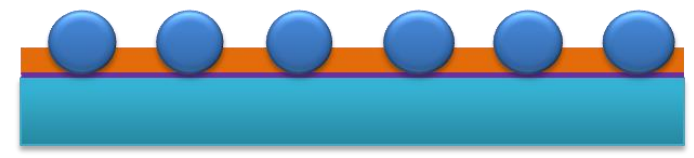

(f) Microsphere Removal

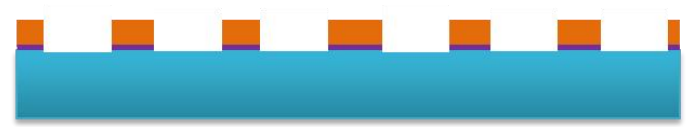

(g)

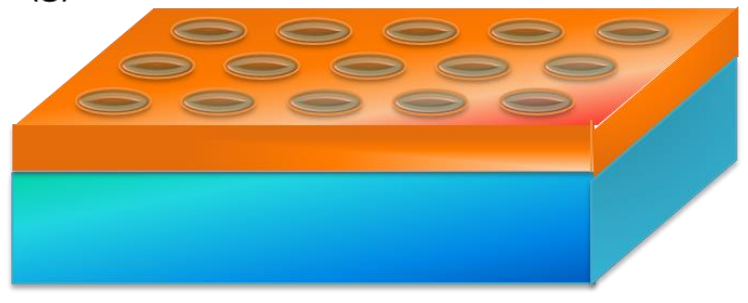

Figure 3.1 Protocol of nanosphere lithography for Au nanohole arrays fabrication. (a) Substrate (quartz slides) cleaning using acid piranha at $90{ }^{\circ} \mathrm{C}$ for two hours and sonicated in acetone, methanol, and D.I. 
water; (b) a monolayer of polystyrene was pattern on the cleaned substrate; (c) etch the PS spheres using Oxygen Plasma Asher; (d) a layer of $5 \mathrm{~nm}$ Ti was deposited; (e) a layer of $45 \mathrm{~nm} \mathrm{Au}$ was deposited; (f)

PS spheres were removed by sonication in methanol; (g) schematic Au nanohole arrays.
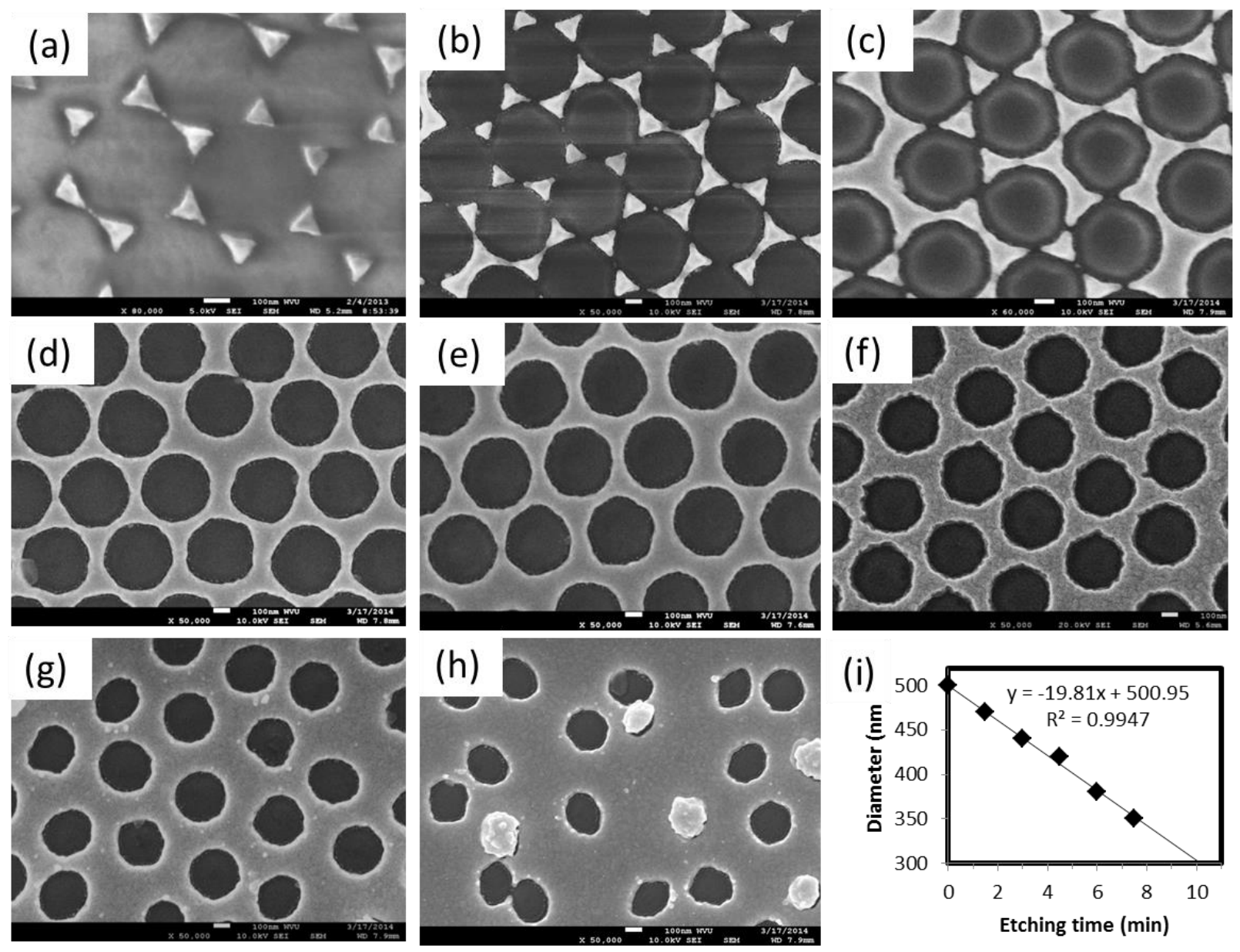

Figure 3.2 (a) to (h) corresponds to Au nanoarrays with a periodicity of $500 \mathrm{~nm}$ and hole diameters of 500 nm, 470 nm, 440 nm, 410 nm, 380 nm, 350 nm, 320 nm, 290 nm. (i) Hole diameter decreases linearly with etching time 

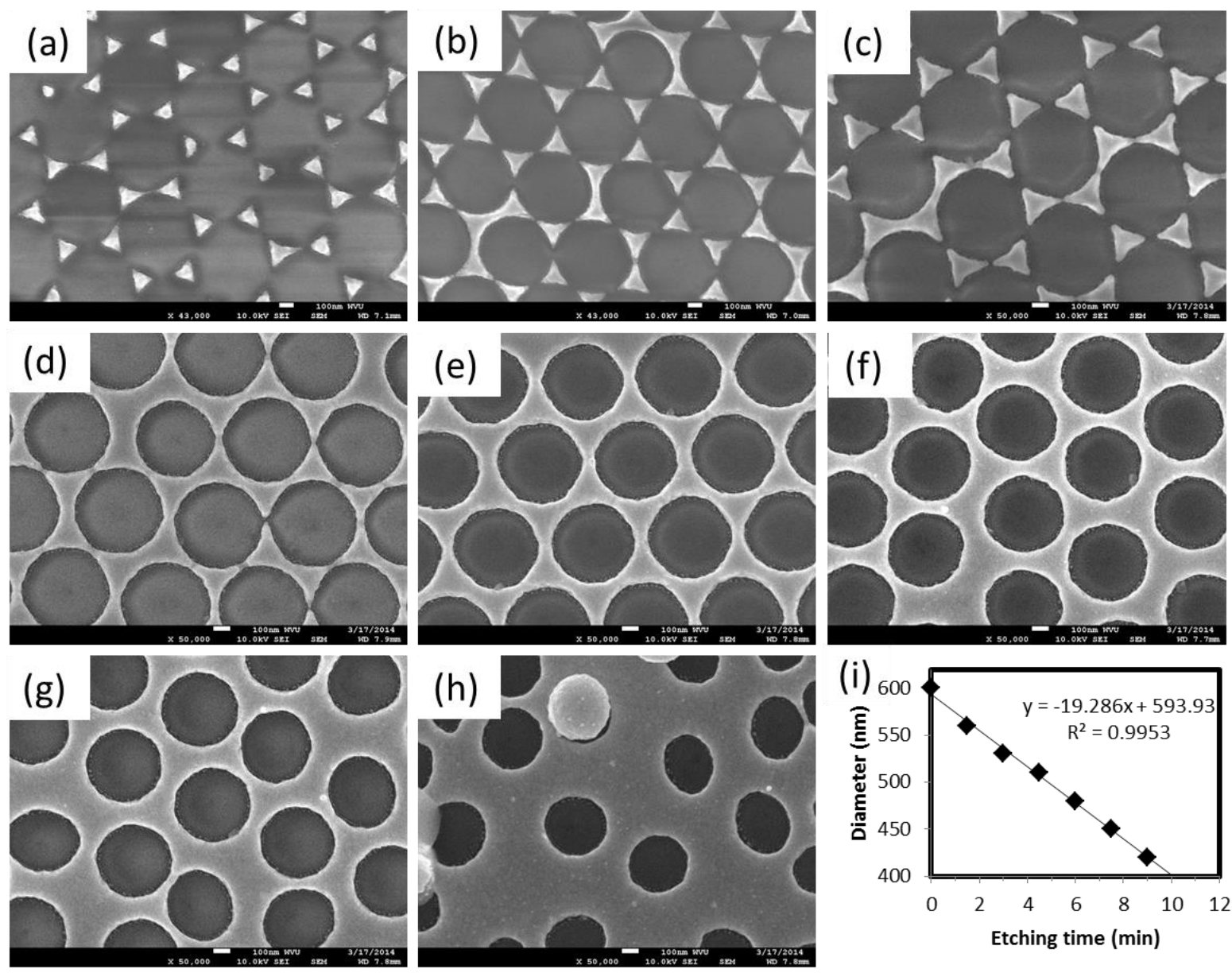

Figure 3.3 (a) to (h) corresponds to Au nanoarrays with a periodicity of $600 \mathrm{~nm}$ and hole diameters of $600 \mathrm{~nm}, 570 \mathrm{~nm}, 540 \mathrm{~nm}, 510 \mathrm{~nm}, 480 \mathrm{~nm}, 450 \mathrm{~nm}, 420 \mathrm{~nm}, 390 \mathrm{~nm}$. (i) Hole diameter decreases linearly with etching time.

\subsubsection{Measuring the refractive index sensitivity of Au nanoarrays}

To measure the refractive index sensitivity glycerine-water mixtures were prepared to make a series of solutions with refractive indices ranging from 1.33333 (only water) to 1.41299 (60\% glycerine in glycerine-water mixture). The shift in the resonances of the Au nanoarrays was measured using a UV-Vis spectrometer for each refractive index solution. The resonance peaks were found to scale linearly with the refractive index increment. The refractive index sensitivity 
for each Au nanoarray was defined as the ratio of the slope of peak shift verse refractive index curve.

\subsubsection{SERS response of Au nanoarrays}

Thiophenol (TP) has four prominent Raman peaks at $997 \mathrm{~cm}^{-1}, 1020 \mathrm{~cm}^{-1}, 1071 \mathrm{~cm}^{-1}$, and 1571 $\mathrm{cm}^{-1}$ which were used to study the SERS response of the as-prepared Au nanoarrays. To coat a uniform layer of the TP molecules on the surface of the Au nanoarrays, TP was first prepared in ethanol with a concentration of $3 \mathrm{mM}$, and then the Au nanoarrays chips were immersed in the solution for two days. The chips were washed by ethanol and dried under compressed nitrogen gas, and after this the Raman measurements was taken.

\subsubsection{FDTD simulations of Au nanotriangle/nanohole arrays}

Finite Difference Time Domain (FDTD) was conducted to study the electromagnetic field distribution and to calculate the transmission of the Au nanotriangle/nanohole arrays using the commercially available Optiwave software ${ }^{102}$. The simulation cell with a grid size of $3 \mathrm{~nm}$ was constructed to match the $\mathrm{Au}$ nanohole arrays fabricated. For full spectrum, the incident wavelengths were from $400 \mathrm{~nm}$ to $1200 \mathrm{~nm}$; for monochromatic light, $785 \mathrm{~nm}$ incident light was used to match the laser used in SERS measurement. The refractive indices of gold and titanium were referred to the data of Palik ${ }^{103}$. The quartz slides were given a refractive index of 1.53 . Gaussian pulse was used as the input wave and linearly polarized in both $\mathrm{x}$ and $\mathrm{y}$ direction. All the simulations were subject to periodic boundary conditions to replicate the periodic gold nanohole arrays.

\subsection{Results and discussion}




\subsubsection{Angle-resolved optical study}

The angle resolved properties of the Au nanohole arrays were first investigated by FDTD simulations. This was done to help clarify which peaks in the optical spectrum corresponded to the LSPR and SPP modes. This is possible because SPP modes display a transmission maximum or reflection minimum which changes with incident angle due to the changing momentum matching conditions. In contrast, LSPR modes show a transmission minimum or reflection maximum that is independent of the incident angle as no momentum matching is necessary for excitation. Therefore by looking at the angular dependence the resonance modes of the $\mathrm{Au}$ nanohole arrays fabricated, as shown in Figure 3.4, were easily determined. For Au nanohole arrays with a periodicity of $500 \mathrm{~nm}$, a hole diameter of $350 \mathrm{~nm}$, and a film thickness of $50 \mathrm{~nm}$, the SPP mode is located at around $800 \mathrm{~nm}$ under normal incidence and the LSPR mode is located at around $680 \mathrm{~nm}$. For Au nanohole arrays with a periodicity of $600 \mathrm{~nm}$, a hole diameter of 420 $\mathrm{nm}$, and a film thickness of $50 \mathrm{~nm}$, the SPP mode is around $940 \mathrm{~nm}$ under normal incidence and the LSPR mode is found to be at around $780 \mathrm{~nm}$. The difference of wavelengths at which the resonance modes occur is caused by the periodicity difference. Au nanohole arrays with larger periodicity supply less momentum making the excited SPP resonance mode red-shift. 
(a)
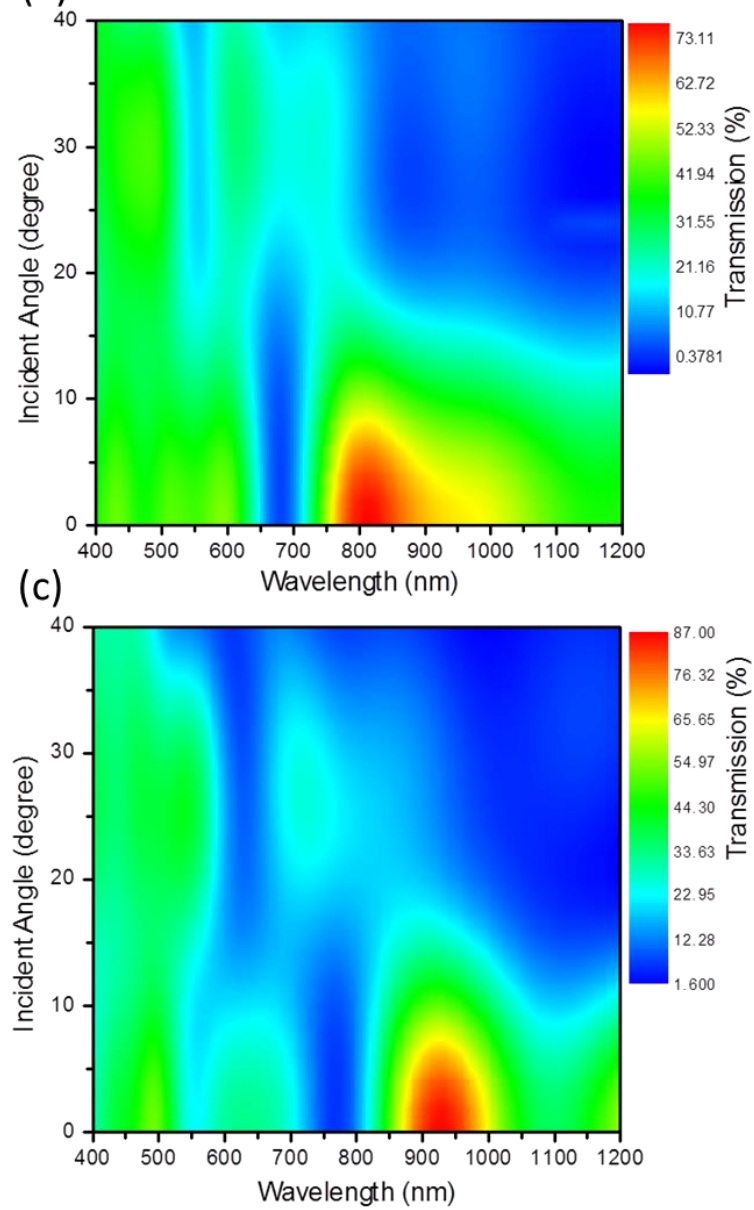

(b)
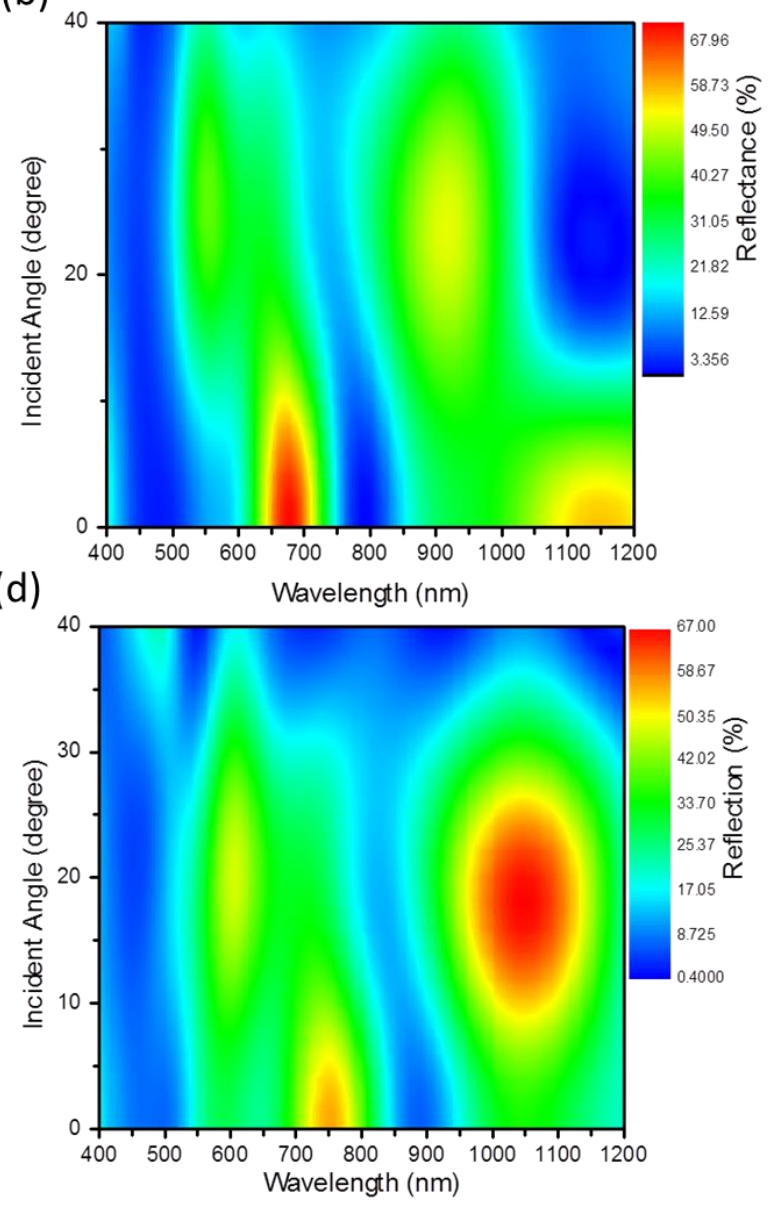

Figure 3.4 Simulated angle-resolved transmission \& reflection spectra for Au nanohole arrays with a periodicity of $500 \mathrm{~nm}((\mathbf{a})$ and (b)), hole diameter of $350 \mathrm{~nm}$, and film thickness of $50 \mathrm{~nm}$, and $600 \mathrm{~nm}$ ((c) and (d)), hole diameter of $420 \mathrm{~nm}$, and film thickness of $50 \mathrm{~nm}$. The incident angle is defined as the angle between the incident light and the direction normal to the surface.

\subsubsection{LSPR evolution}

Au nanotriangle arrays existed for short etching times. The Au nanotriangle arrays are composed of individual isolated triangular nanoparticles arranged in a hexagonal manner. The transmission minimum measured in Figure 3.5 is attributed to the LSPR absorption. The LSPR modes is highly dependent on the size of the nanoparticle ${ }^{104}$. For smaller nanoparticles, dipolar resonances 
are excited. An increase of the size leads to higher order resonance modes (quadrupole, octupole, etc.) being excited. As the nanoparticle size increases, the redshift of the LSPR can be thought of as corresponding to how strongly the charge oscillations are localized. In small nanoparticles, the oscillating charge is tightly confined compared to the wavelength of the plasmon. As the size increases, the oscillations are increasing at the surface, and less resistance is felt to the oscillating charges, red-shifting the resonance condition.

Shown in Figure 3.5 is the evolution of LSPR mode from no etching to 10.5 min etching with a step of 1.5 min etching. For Au nanoarrays with a periodicity of $500 \mathrm{~nm}$, the resulting hole diameters are around $500 \mathrm{~nm}, 470 \mathrm{~nm}, 440 \mathrm{~nm}, 410 \mathrm{~nm}, 380 \mathrm{~nm}, 350 \mathrm{~nm}, 320 \mathrm{~nm}$, and $290 \mathrm{~nm}$, respectively. For Au nanoarrays with a periodicity of $600 \mathrm{~nm}$, the hole diameters after etching are around $600 \mathrm{~nm}, 570 \mathrm{~nm}, 540 \mathrm{~nm}, 510 \mathrm{~nm}, 480 \mathrm{~nm}, 450 \mathrm{~nm}, 420 \mathrm{~nm}$, and $390 \mathrm{~nm}$, respectively. 

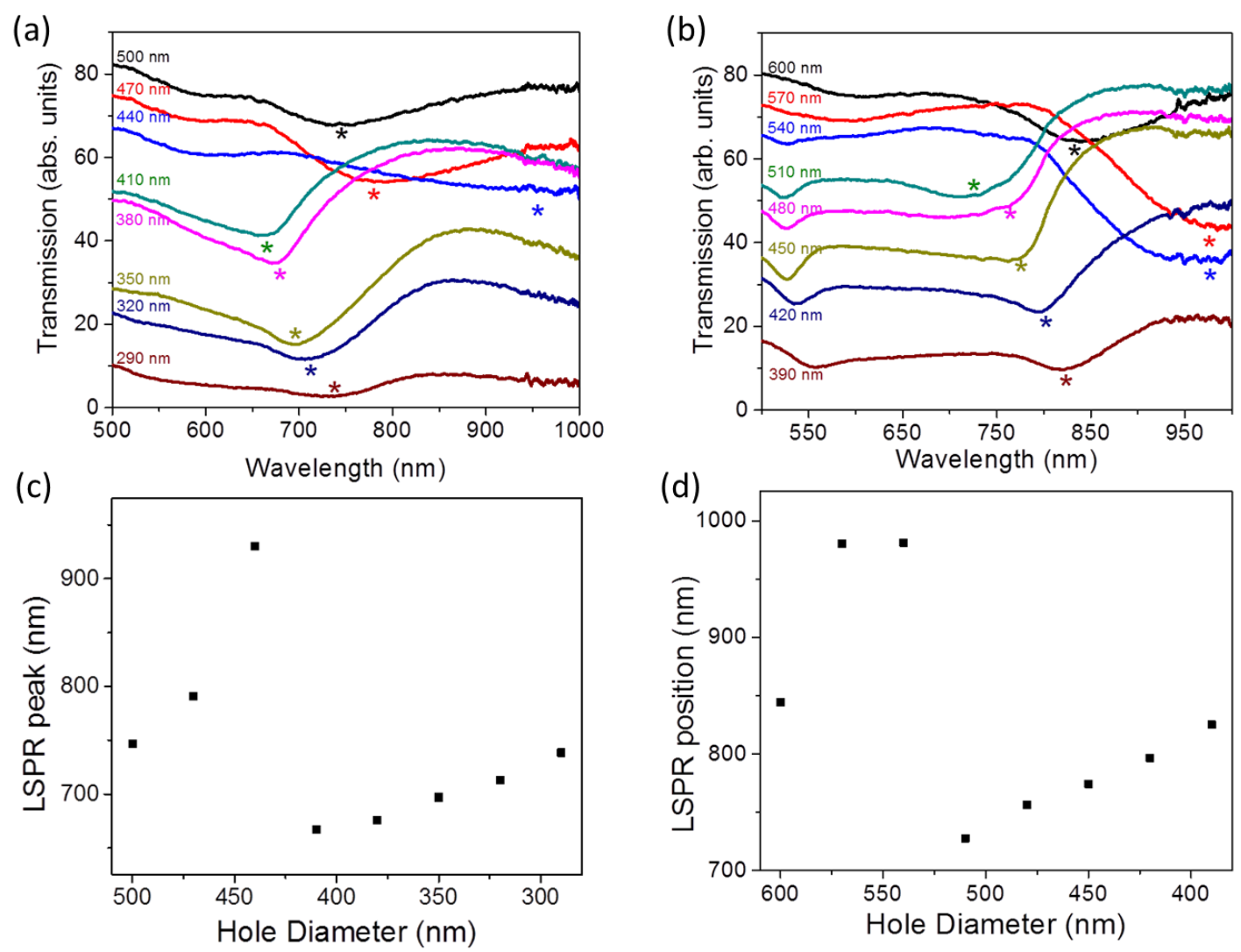

Figure 3.5 Experimental UV-Vis spectra for Au nanoarrays with a periodicity of (a) $500 \mathrm{~nm}$ and (b) 600 $\mathrm{nm}$. The star $(*)$ shows LSPR positions in (c) with a periodicity of $500 \mathrm{~nm}$ and (d) with a periodicity of $600 \mathrm{~nm}$.

The LSPR peak shifts in two distinct regions. First, when the etching time is small and nanotriangles still exist, the peak redshifts with increasing size. Although the resonance will blue shift for fixed size triangles as the gap distance decreases, this effect is not seen due to the overall increase in triangle size ${ }^{104}$. Secondly, Figure 3.5 shows that during the transition from nanotriangle to nanohole array the peak position blueshifts drastically (smaller than $410 \mathrm{~nm}$ for $\mathrm{Au}$ nanohole arrays with a periodicity of $500 \mathrm{~nm}$ and smaller than $510 \mathrm{~nm}$ for Au nanohole arrays with a periodicity of $600 \mathrm{~nm}$ ). Further decrease of hole diameters leads to a continuous 
and steady red shift of the transmission minimum. The dramatic blueshifts of LSPR are caused by the sudden change of the shape from nanotriangle arrays to nanohole arrays.

In the nanotriangle region, the LSPR resonance behaves in nanoparticle-like fashion, with the redshift corresponding to increased nanotriangle size. In the nanohole array region, the LSPR resonance of the nanoholes is complementary to that of the nanoparticles of the same shape, related by ${ }^{20} w_{\text {hole }}^{2}+w_{\text {particle }}^{2}=w_{p}^{2}$, where $w_{\text {hole }}$ is the LSPR frequency of nanoholes, $w_{\text {particle }}$ is the LSPR frequency of nanoparticles that have the same shape as the nanoholes, and $w_{p}$ is the bulk plasma frequency. Therefore, when Au nanohole arrays just form, the large-size of the nanohole corresponds to a large nanoparticle which has a redshifted frequency, and the Au nanohole must have a more blue-shifted resonance. The switch from localized surface plasmon in the nanoparticle to localized surface plasmon resonance in the void is the reason for the dramatic switch in the transition region. The continuous red shift of the LSPR in the Au nanohole array region after this point can therefore be interpreted complimentary to the blueshift of the LSPR for a nanoparticle of decreasing size.

\subsubsection{Refractive index sensitivity}

To better understand the plasmonic properties in the transition from Au nanotriangle arrays to Au nanohole arrays, the refractive index sensitivity was measured. A strong correlation between the refractive index sensitivity and the hole diameters (see Figure 3.6) was found, with triangular shaped $\mathrm{Au}$ nanoparticle arrays displaying a lower refractive index sensitivity than the $\mathrm{Au}$ nanohole arrays. The transition zone as shown in Figure 3.5 corresponds to steadily increasing refractive index sensitivity. Once the nanohole is formed, the sensitivity to the refractive index remains a constant. The range over which the refractive index sensitivity increases corresponds 
to the region over which the LSPR was most sensitive to changes in topology, linking the two experiments and revealing the region for which the confined electromagnetic field is most susceptible to the local environment.

The refractive index sensitivity data missing in Figure 3.6 (a) for the Au nanohole array with a periodicity of $500 \mathrm{~nm}$ and a hole diameter of $440 \mathrm{~nm}$ is due to the LSPR shifting outside of the wavelength range of the UV-Vis spectrometer. The data for the Au nanohole array with a periodicity of $500 \mathrm{~nm}$ and a hole diameter of $290 \mathrm{~nm}$ is also missing because the LSPR merges with a SPP mode originating from the interface between the Au nanohole array and the quartz substrate, making peak shift undeterminable.

(a)

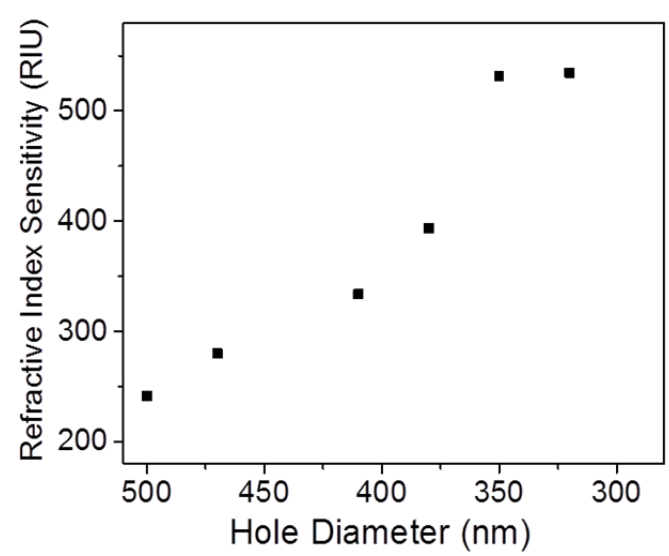

(b)



Figure 3.6 Experimental refractive index sensitivity for Au nanoarrays with a periodicity of (a) $500 \mathrm{~nm}$ and (b) $600 \mathrm{~nm}$.

\subsubsection{Hole size dependent resonance modes}

FDTD simulations were used to verify the experimentally measured hole size dependent resonances and gain insight into the changes seen in Figure 3.7. The size of nanoholes were changed from $500 \mathrm{~nm}$ to $290 \mathrm{~nm}$ with a step of $30 \mathrm{~nm}$ for nanoarrays with a periodicity of 500 
$\mathrm{nm}$, and changed from $600 \mathrm{~nm}$ to $390 \mathrm{~nm}$ also with a step of $30 \mathrm{~nm}$ for nanoarrays with a periodicity of $600 \mathrm{~nm}$. In the nanotriangle array region only the LSPR is measured. In the nanohole region, both SPP and LSPR co-exist, necessitating FDTD simulations to further confirm the origin of each mode. The simulation results show the Au nanohole array optical properties beginning when the hole diameter is equal or smaller than $410 \mathrm{~nm}$ for a periodicity of $500 \mathrm{~nm}$ and equal or smaller than $510 \mathrm{~nm}$ for a periodicity of $600 \mathrm{~nm}$. The LSPR modes were found to linearly shift to the longer wavelength with a decrease in the hole diameter, which confirmed the experimental results. Similar to LSPR, the SPP also shifts to the red in response to the hole size decrease. Although from the diffraction condition the hole size should not affect the periodicity and the SPP should remain at a constant wavelength, due to a weak scattering by small holes, SPP is less efficiently excited and thus slightly red-shifts with a decrease of the hole size. 
(a)
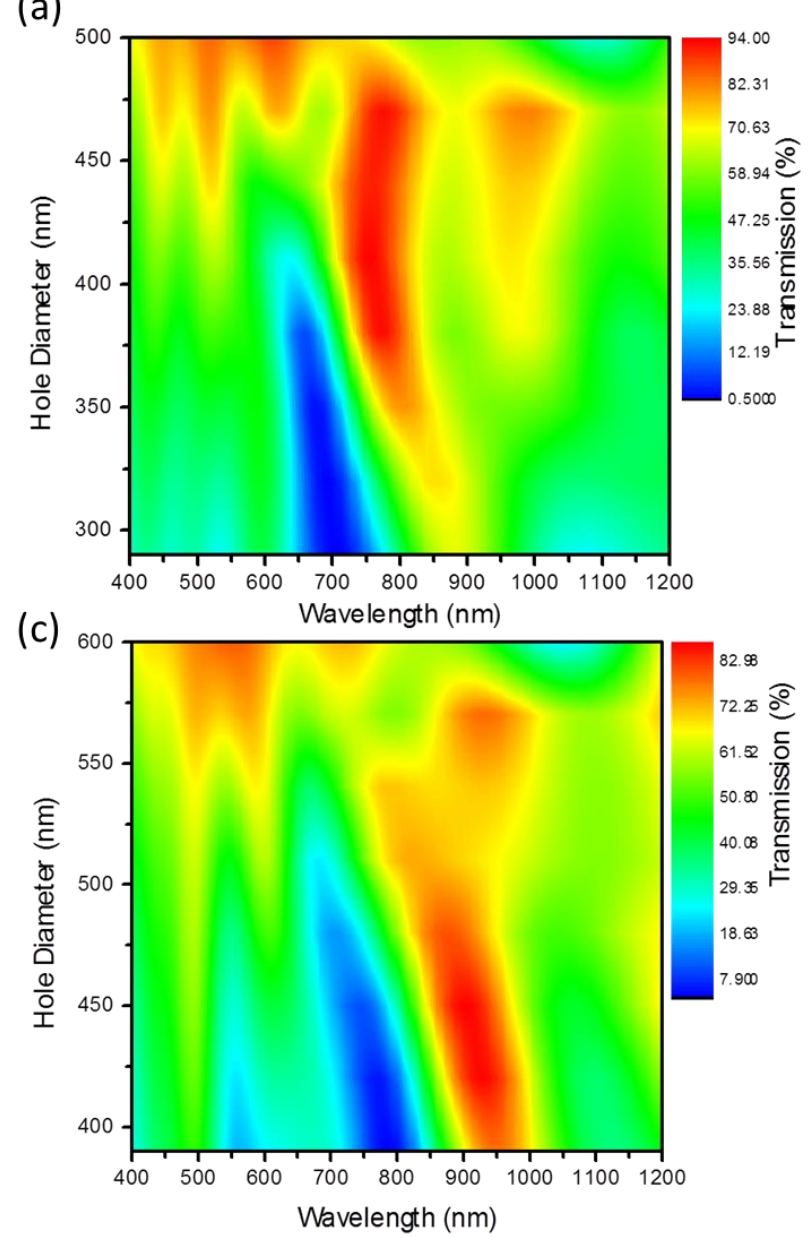

(b)
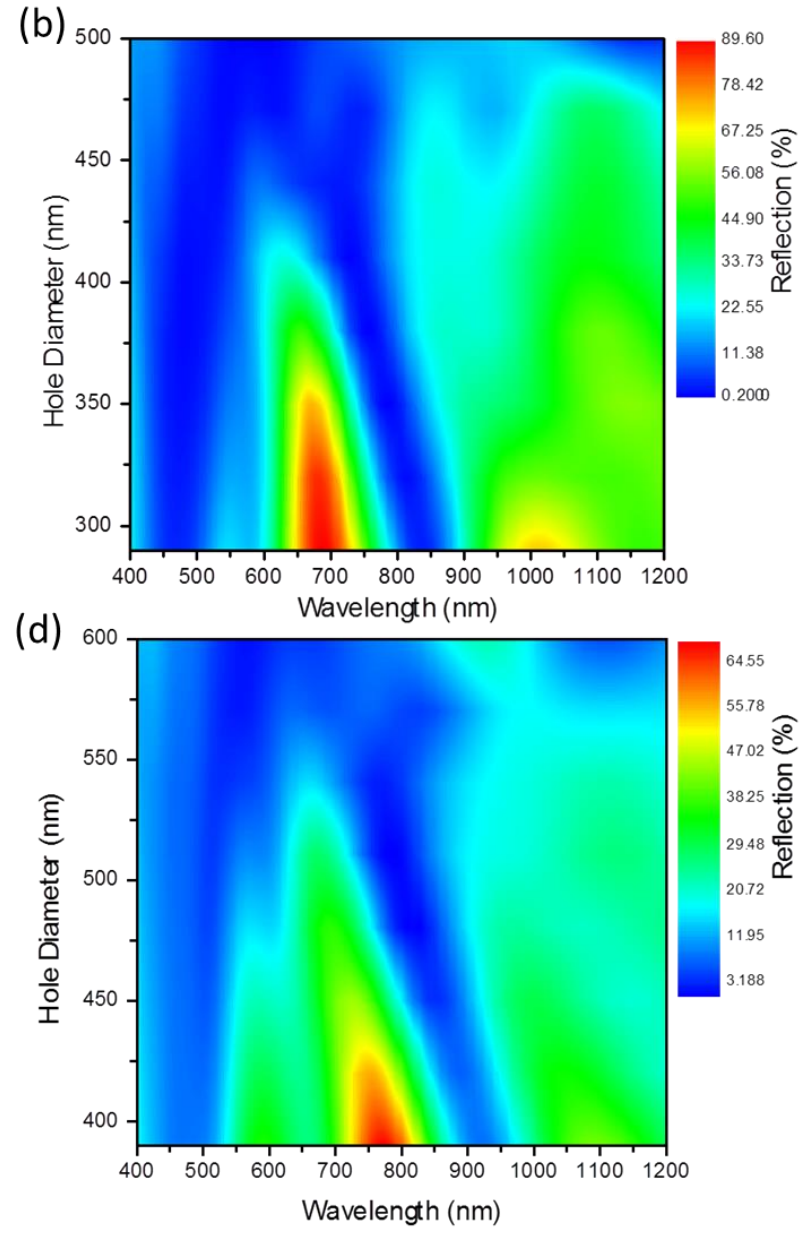

Figure 3.7 Simulated UV-Vis spectra of Au nanoarrays with (a) a periodicity of $500 \mathrm{~nm}$, film thickness of $50 \mathrm{~nm}$, and hole diameters changing from $500 \mathrm{~nm}$ to $290 \mathrm{~nm}$ with a step of 30 (b) a periodicity of 600 $\mathrm{nm}$, film thickness of $50 \mathrm{~nm}$, and hole diameters changing from $600 \mathrm{~nm}$ to $390 \mathrm{~nm}$ with a step of $30 \mathrm{~nm}$

\subsubsection{Au film thickness dependent resonance modes}

FDTD simulations were also conducted to investigate how the resonance modes changed with the Au film thickness, shown in Figure 3.8. For Au nanohole arrays with a periodicity of $500 \mathrm{~nm}$ and a hole diameter of $350 \mathrm{~nm}$, both LSPR and SPP modes were found to remain unchanged when the Au film thickness changed from $100 \mathrm{~nm}$ to $40 \mathrm{~nm}$. With a film thickness of less than 40 $\mathrm{nm}$, the SPP modes on the two interfaces of the Au nanohole arrays interacted with each other. A 
strong interaction occurred with further decreasing thickness, which split the SPP frequencies into two branches which corresponded to low frequency mode (symmetric) and high frequency mode (antisymmetric) ${ }^{17}$. Figure 3.8 (a) revealed that the SPP resonance mode with a wavelength of around $800 \mathrm{~nm}$ split into a low frequency mode of around $1000 \mathrm{~nm}$ and a high frequency mode of about $600 \mathrm{~nm}$ when the metallic film thickness reduced to $10 \mathrm{~nm}$. The other low frequency mode shifted to about $1150 \mathrm{~nm}$ and high frequency mode to around $700 \mathrm{~nm}$ for original resonance at $940 \mathrm{~nm}$ in Figure 3.8 (c).

(a)



(c)

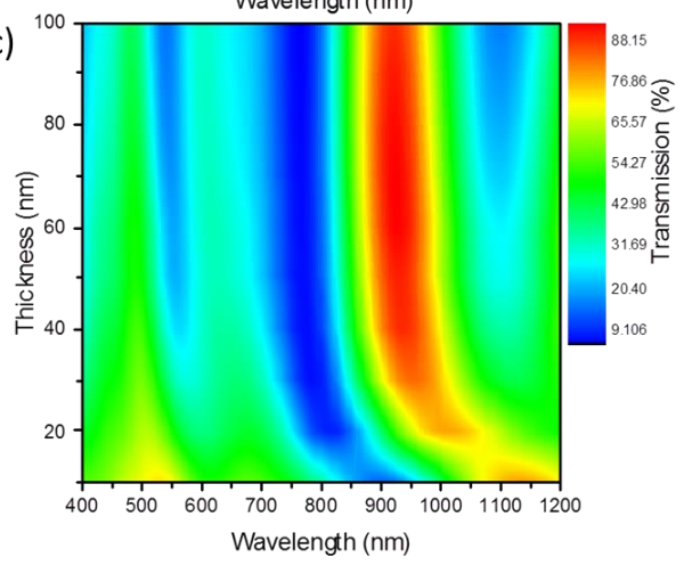

(b)
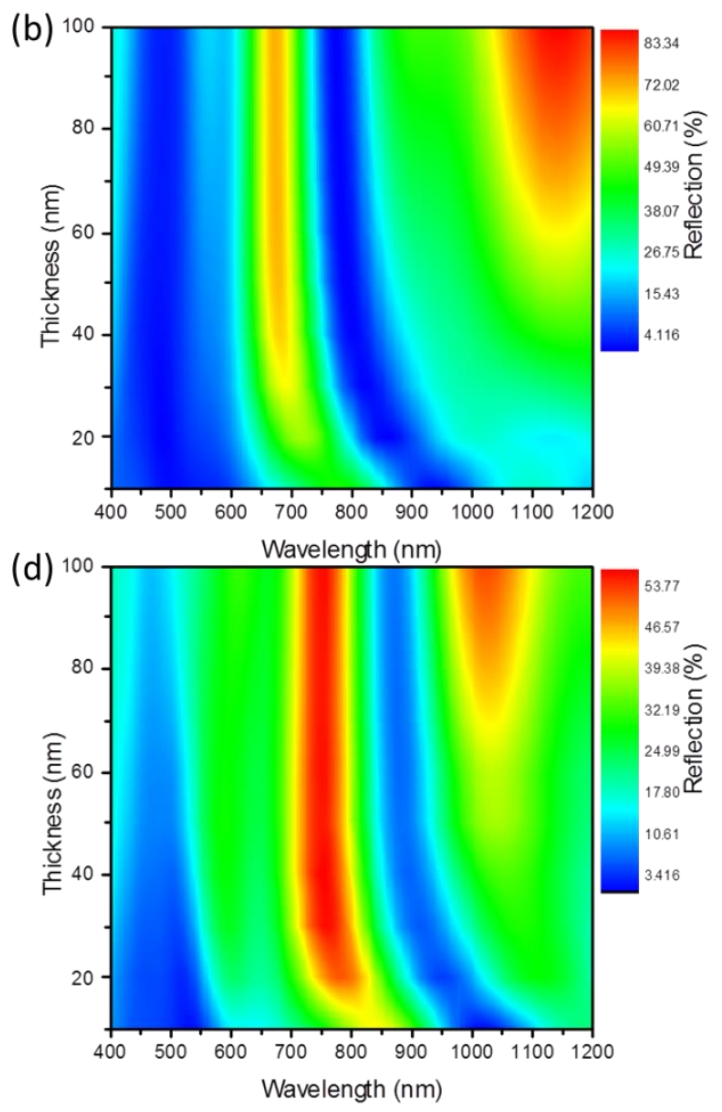

Figure 3.8 Simulated UV-Vis spectra of Au nanohole arrays with (a) and (c) a periodicity of $500 \mathrm{~nm}$, hole diameter of $350 \mathrm{~nm}$, and film thickness changing from $10 \mathrm{~nm}$ to $100 \mathrm{~nm}$ with a step of $10 \mathrm{~nm}$ (b) and (d) a periodicity of $600 \mathrm{~nm}$, hole diameter of $420 \mathrm{~nm}$, and film thickness changing from $10 \mathrm{~nm}$ to $100 \mathrm{~nm}$ with a step of $10 \mathrm{~nm}$. 


\subsubsection{Periodicity dependent resonance modes}

The periodicity of the Au nanohole arrays was also found to highly influence the resonance modes (Figure 3.9). First, the periodicity was changed from $100 \mathrm{~nm}$ to $1000 \mathrm{~nm}$ while the ratio of hole diameter to the periodicity was kept constant at 0.7 and the Au film thickness was kept constant at 50nm. Second, the periodicity was changed from $400 \mathrm{~nm}$ to $1000 \mathrm{~nm}$ but the hole diameter and Au film thickness were kept constant at $350 \mathrm{~nm}$ and $50 \mathrm{~nm}$, respectively. In both cases, increasing the periodicity consistently resulted in a red shift of both the SPP and LSPR resonance modes. Compared to the hole size and film thickness, the periodicity led to larger shifts in the resonance modes. A red shift of SPP is caused by the decrease of the extra momentum supplied due to increasing periodicity making the coupling between incident light and the surface plasmon less efficient. At the same time, the hole-to-hole separation was enlarged at an increase of the periodicity. The LSPR resonance modes became less energetic as a result of the increasingly isolated nanoholes and finally became red-shifted. 

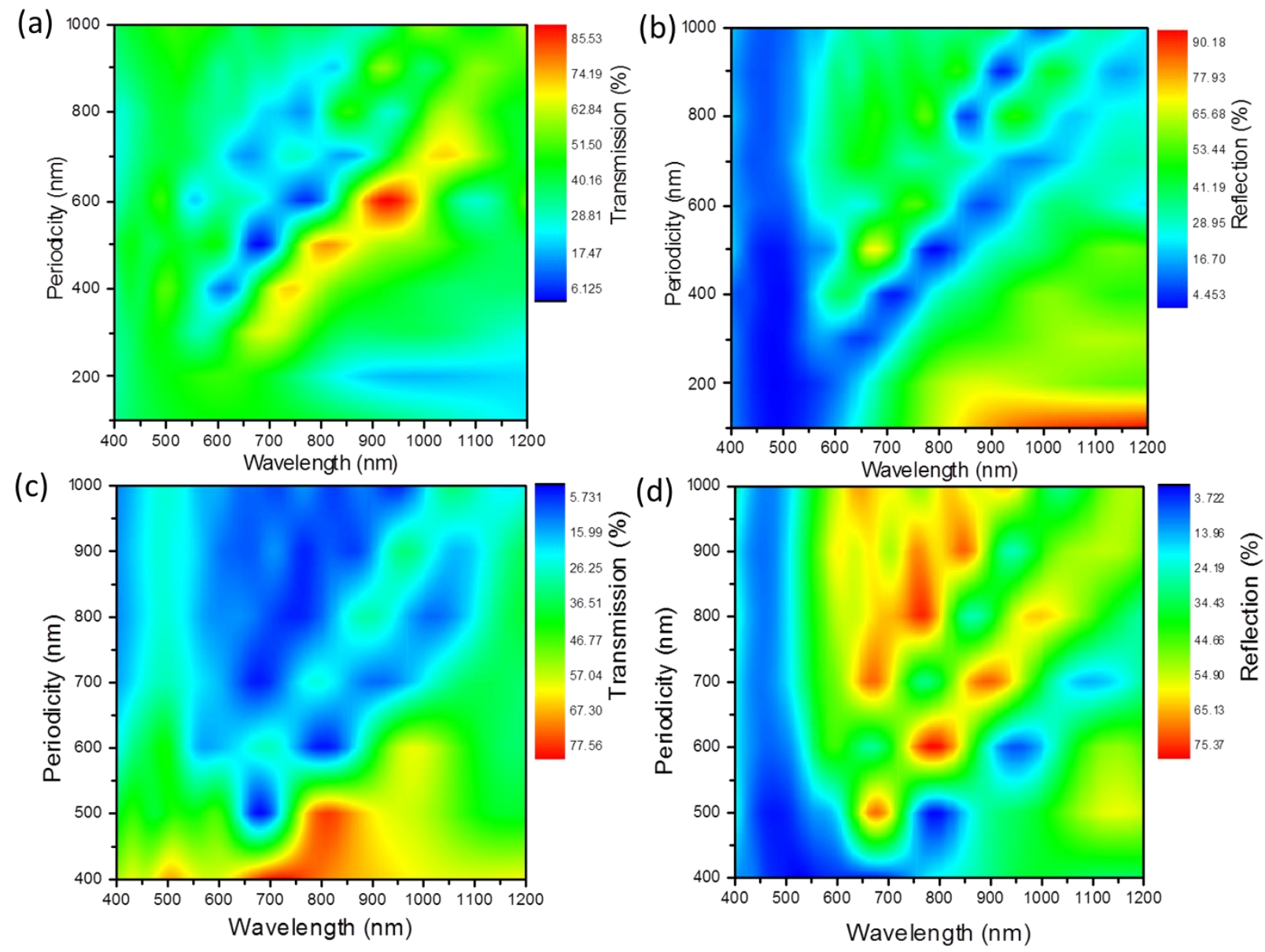

Figure 3.9 Simulated UV-Vis spectra of Au nanohole arrays with varying periodicities from 100 to 1000 nm. (a) and (b): the ratio of hole diameter to periodicity is kept constant at 0.7 and the Au film thickness remains at $50 \mathrm{~nm}$; (c) and (d): the hole diameter and Au film thickness remain constant at $350 \mathrm{~nm}$ and 50 nm, respectively.

\subsubsection{SERS response of Au nanoarrays}

The goal of this Chapter is to gain an understanding of how Au nanoarrays with different hole diameters contribute to SERS performance. Both Au nanotriangle arrays and Au nanohole arrays were previously found to enhance SERS. However, no study has systematically clarified the correlation between SERS performance and the optical properties of the two resonance modes in 
the Au arrays. There are several possible contributions to the SERS enhancement with the mixed resonance modes.

First, the local EM field is highly concentrated due to the LSPR. In the Au nanohole array the rim area of each individual hole is where the localized field is most intensively concentrated. For Au nanotriangle arrays, the triangle tips and the connecting area of the bow-tie nanostructure are where the hot spots are most likely to be found.

Second, SEM images (Figure 3.10 (b) and Figure 3.11 (b)) reveal that fabricated Au nanoarrays have defects. These defects exist because of the mask being damaged during etching, forming dimers or trimers of nanoholes and leading to cracks between connected triangles. These defects act to further concentrate the local EM field beyond that possible if perfect order is assumed.

Third, significant reflectance from the Au nanohole arrays (Figure 3.7 (b) and (d)) can also play a role in enhancing the SERS signal. For $\mathrm{Au}$ nanotriangle arrays, the LSPR leads to little reflection. For Au nanohole arrays, the film area increases with a decrease of the hole diameter, which makes the reflectance significant. As the laser with an excitation wavelength of $785 \mathrm{~nm}$ does not overlap with each of the Au nanoarrays studied, resonant excitation of either LSPR or SPP mode is unlikely to have involved in the strong reflectance, which is more likely to be caused by the increasingly large gold film area with a decrease of hole diameters. Under strong reflectance condition, the incident light has a higher probability of being scattered and the Raman signal has a higher probability of being reflected back to the detector, increasing the signal.

The combination of these three factors is seen in the experimental Raman data. The Raman response from two kinds of Au nanoarrays with varying hole diameters is shown in Figure 3.10 
and Figure 3.11: one with a periodicity of $500 \mathrm{~nm}$, a film thickness of $50 \mathrm{~nm}$, and hole diameters changing from $500 \mathrm{~nm}$ to $290 \mathrm{~nm}$ with a step of $30 \mathrm{~nm}$; the other with a periodicity of $600 \mathrm{~nm}$, a film thickness of $50 \mathrm{~nm}$ and hole diameters changing from $600 \mathrm{~nm}$ to $390 \mathrm{~nm}$ with a step of 30 $\mathrm{nm}$. The four prominent peaks from thiophenol at $997 \mathrm{~cm}^{-1}, 1020 \mathrm{~cm}^{-1}, 1071 \mathrm{~cm}^{-1}$, and $1571 \mathrm{~cm}^{-1}$ were used to gauge the SERS enhancement. For Au nanohole arrays with a periodicity of $500 \mathrm{~nm}$, as shown in Figure 3.10 (a), the Raman intensity increased steadily when the hole diameter dropped below $500 \mathrm{~nm}$ and reached the maximum value when the hole diameter decreased to $440 \mathrm{~nm}$. Below $440 \mathrm{~nm}$, there was a sudden drop in intensity but it gradually increased again until $350 \mathrm{~nm}$, after which the intensity again decreased. For the $600 \mathrm{~nm}$ periodicity hole array, the measured Raman intensity first decreased when the hole diameter decreased from $600 \mathrm{~nm}$ then increased steadily until at a hole diameter of $510 \mathrm{~nm}$.

The trend in Raman enhancement at both periodicities is found to follow a combination of the reflection, local field without disorder, and local field with disorder as shown in Figure $\mathbf{3 . 1 0}$ and Figure 3.11. A linear combination of each contribution was compared to the normalized Raman intensity at $1071 \mathrm{~cm}^{-1}$, with the same contributions describing the data for both periodicities. This data proves the mixed SPP and LSPR response of the Au nanoarrays can be decomposed into three dominant factors. 
(a)

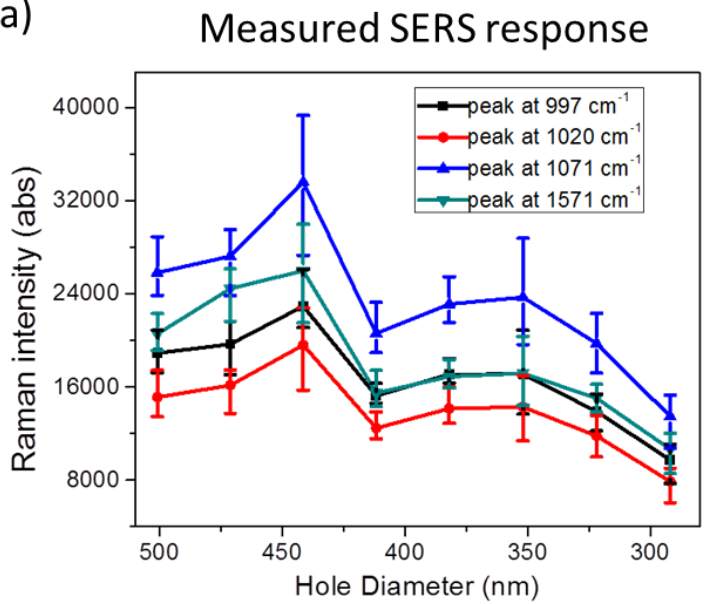

(c)

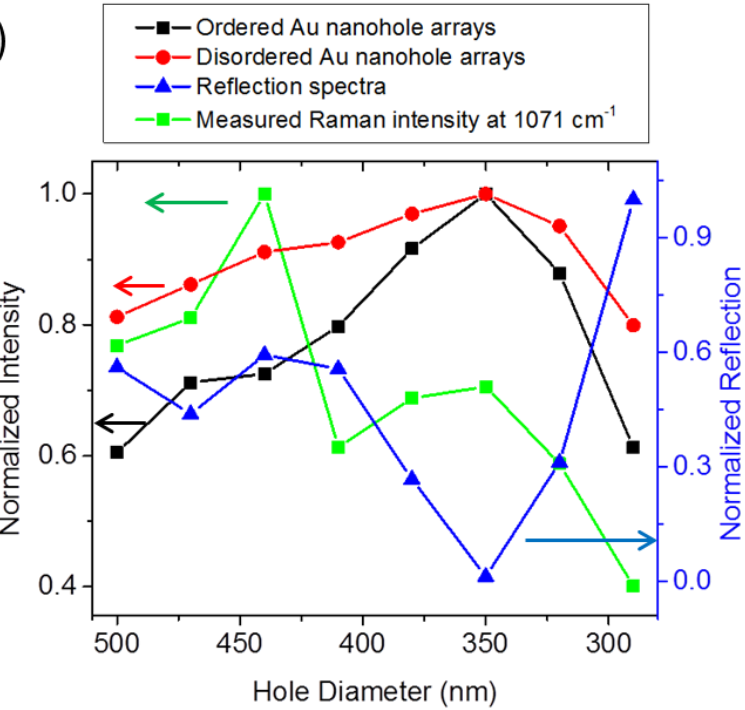

(b)

Low magnification SEM images

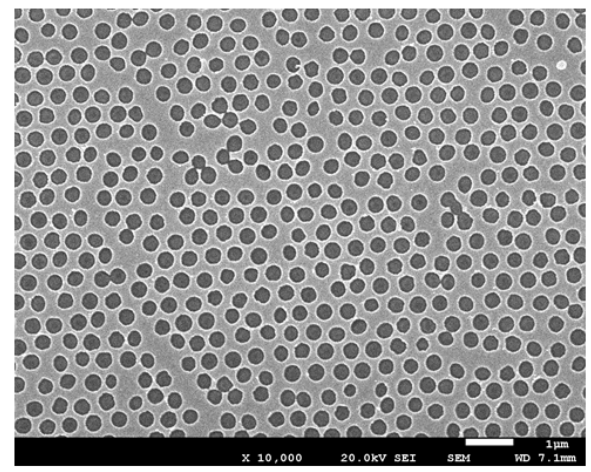

(d)

Calculated data comparison

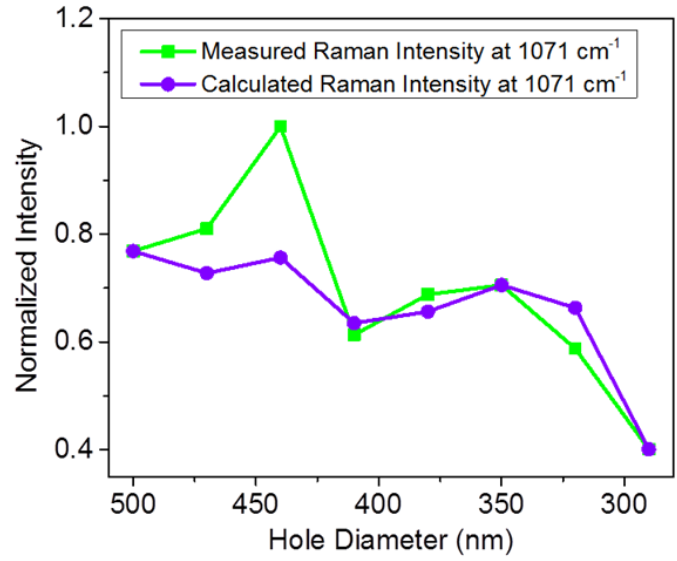

Figure 3.10 (a) Experimentally measured SERS response of Au nanoarrays with a periodicity of $500 \mathrm{~nm}$, a film thickness of $50 \mathrm{~nm}$, and hole diameters changing from $500 \mathrm{~nm}$ to $290 \mathrm{~nm}$ with a step of $30 \mathrm{~nm}$. The

Raman signals were acquired under 785 nm laser from thiophenol (TP) which was coated on $\mathrm{Au}$ nanoarrays. Four prominent peaks of TP were plotted in the figure. (b) SEM image of Au nanohole arrays with a periodicity of $500 \mathrm{~nm}$, a film thickness of $50 \mathrm{~nm}$, and a hole diameter of $350 \mathrm{~nm}$. It reveals that experimentally fabricated Au nanohole arrays are not in perfect order. The defects may have an influence on the overall Raman performance. (c) A comparison of simulated electric field enhancement factors of ordered and disordered Au nanoarrays, simulated reflection spectra, and measured Raman intensity on Au nanoarrays with a periodicity of $500 \mathrm{~nm}$, a film thickness of $50 \mathrm{~nm}$, and hole diameter changing from 500 $\mathrm{nm}$ to $290 \mathrm{~nm}$ with a step of $30 \mathrm{~nm}$. Both the defects and strong reflections of Au nanoarrays were taken 
into account in understanding the factors that contribute to the overall Raman performance. (d) A comparison of the measured Raman intensity and the calculated Raman intensity. For the calculated Raman intensity, the defects and reflection were linearly combined with perfect order nanoarrays to give an overall contribution to the Raman performance.
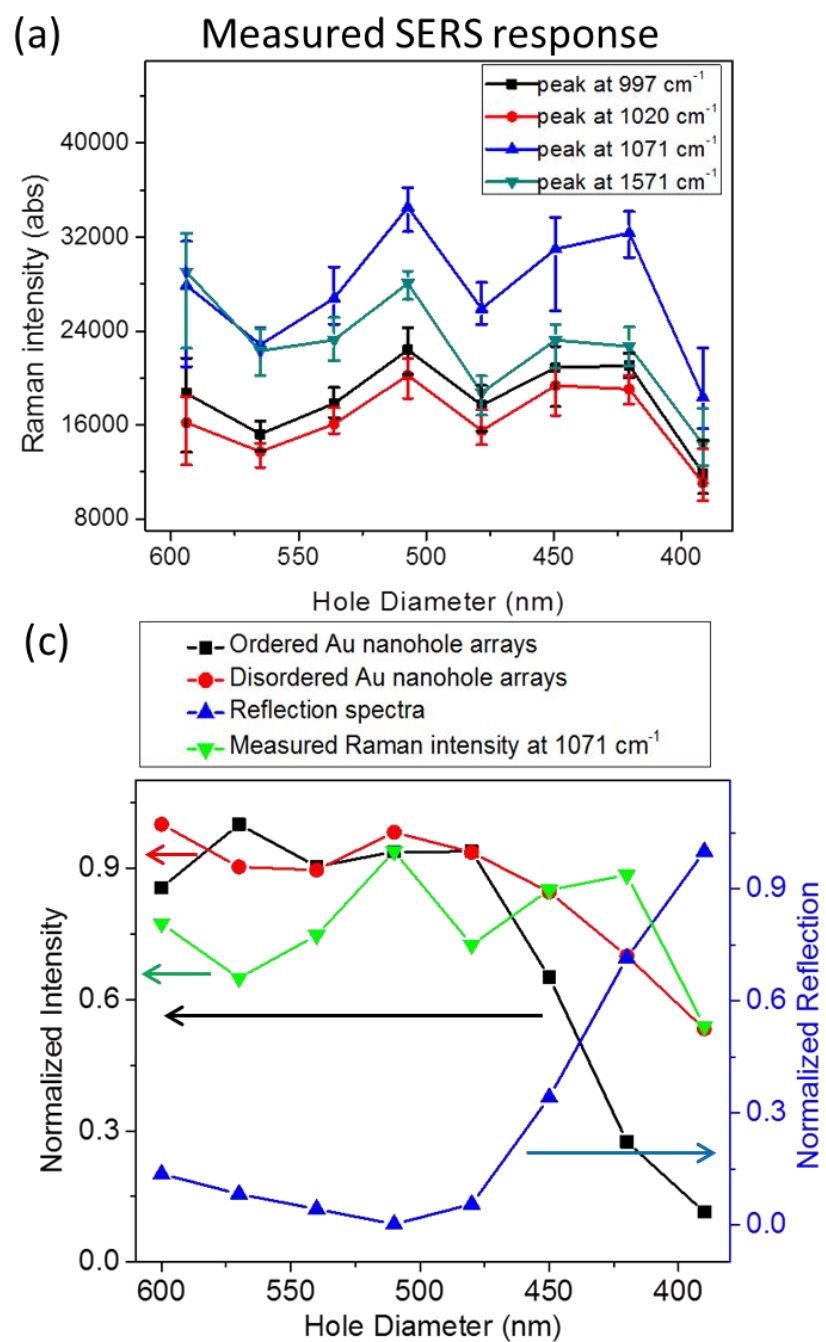

(b)



(d)

\section{Calculated data comparison}

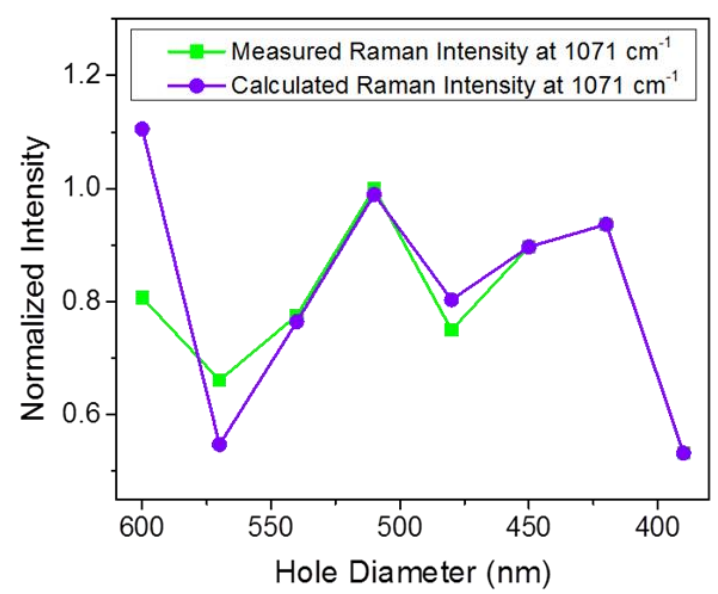

Figure 3.11 (a) Experimentally measured SERS response of Au nanoarrays with a periodicity of $600 \mathrm{~nm}$, a film thickness of $50 \mathrm{~nm}$, and hole diameters changing from $600 \mathrm{~nm}$ to $390 \mathrm{~nm}$ with a step of $30 \mathrm{~nm}$. The

Raman signals were acquired under $785 \mathrm{~nm}$ laser from thiophenol (TP) which was coated on $\mathrm{Au}$ nanoarrays. Four prominent peaks of TP were plotted in the figure. (b) SEM image of Au nanohole arrays with a periodicity of $600 \mathrm{~nm}$, a film thickness of $50 \mathrm{~nm}$, and a hole diameter of $420 \mathrm{~nm}$. It reveals that 
experimentally fabricated Au nanohole arrays are not in perfect order. The defects may have an influence on the overall Raman performance. (c) A comparison of the simulated electric field enhancement factors of ordered and disordered Au nanoarrays, simulated reflection spectra, and measured Raman intensity on Au nanoarrays with a periodicity of $600 \mathrm{~nm}$, a film thickness of $50 \mathrm{~nm}$, and hole diameter changing from $600 \mathrm{~nm}$ to $390 \mathrm{~nm}$ with a step of $30 \mathrm{~nm}$. Both the defects and strong reflections of Au nanoarrays were taken into account in understanding the factors that contribute to the overall Raman performance. (d) A comparison of the measured Raman intensity and calculated Raman intensity. For the calculated Raman intensity, the defects and reflection were linearly combined with perfect order nanoarrays to give an overall contribution to the Raman performance. All the data are normalized.

\subsubsection{Scale Invariant Properties}

Investigations above unraveled a couple of optical properties that are intrinsic to nanoarrays. First, the transition zone from nanotriangle arrays to nanohole arrays occurs when the hole diameter is reduced by $30 \mathrm{~nm}$ from the initial nanotriangle arrays. Second, LSPR resonance modes are independent of the incident angle. Third, in the nanohole array region, LSPR and SPP resonance modes shift to longer wavelength with an increase of the periodicity or with a decrease of hole diameter but remain unchanged when film thickness exceeds $40 \mathrm{~nm}$. Fourth, the local EM field with ordered and disordered nanohole arrays and the reflectance from the metallic film combined to give the final Raman enhancement.

\subsection{Conclusion}

In conclusion, we have systematically investigated the optical properties of Au nanoarrays. The resonance modes supported by Au nanoarrays display a dependence on the hole diameter, film thickness, and the periodicity. The LSPR peak evolutions and the refractive index sensitivity 
study revealed a distinct transition from Au nanotriangle arrays to Au nanohole arrays. Finally, a study of the Raman response over varying hole diameters suggest a synergistic effects from the electric field enhancement of the defect-containing and defect-free nanoarrays together with the strong reflectance of $\mathrm{Au}$ nanoarrays in the overall contribution to the SERS intensity. These results provide guidelines for selecting the proper nanoarray substrate for any SERS sensing application. 


\section{Chapter 4: Gold Nanohole Arrays Based Surface-Enhanced Raman Scattering (SERS) Biosensor for Silver (I) Detection}

\subsection{Introduction}

Metals $^{105}$ such as mercury, lead, chromium, calcium, arsenic, and nickel can be very toxic to humans and in response a series of techniques have been developed for their detection. Recently, silver and silver composites began drawing attention due to increased application in electronics, photographic imaging, and pharmaceutical aspects ${ }^{106,107}$ and possible resulting exposure. Silver in waste products ends up in the aquatic ecosystem, producing toxic silver ions. As well, adverse effects from the release of metal ions, like silver in saliva from dental cast alloys has been recently recognized ${ }^{108}$. While in vitro studies suggest that corrosion of dental alloys contributes to the increase of metal ions in saliva, many in vivo studies fail to confirm the results because of a lack of repeatable measurements. It is imperative to develop a quick and accurate measurement method for metal ions in solution and in saliva.

Among previous detection methods for metal ions, the most popular are atomic absorption spectroscopy, inductively coupled plasma/mass spectrometry, inductively coupled plasma/atomic emission spectrometry, and ultraviolet-visible spectrometry ${ }^{109-112}$. Despite these techniques being sensitive and selective, tedious sample preparation, expensive instrumentation, and professional operation limit application in point of care and limited time measurement. Recently oligonucleotide-based fluorescent sensors ${ }^{106,107,113,114}$ were also proposed with a very low limit of detection (LOD) for metal ions. However, the fluorescent sensors are based on organic dyes, which suffer from photo bleaching, limited sensitivity, and poor reproducibility. Surface Enhanced Raman Scattering (SERS) ${ }^{13}$ has the potential to avoid and/or overcome the common drawbacks of the aforementioned methods, providing an alternative design path for the detection 
of silver ions due to its ultrasensitive detection ability, high reproducibility, molecular spectral fingerprint, easy operation, and on-site detection capability ${ }^{115-117}$.

Noble metals (such as $\mathrm{Au}, \mathrm{Ag}$, and $\mathrm{Cu}$ ) can support strong surface plasmon resonance (SPR) which is tunable by changing the shape, size, and dielectric environment. SPR based sensors have been widely studied due to the straightforward working mechanism and simplicity in detection, however, they suffer from limited sensitivity and are intrinsically lack of selectivity for molecular recognition ${ }^{118}$. Therefore, SERS sensors are often preferred in environmental monitoring and biological detection. LSPR has been heavily studied in enhancing SERS. The large electromagnetic field of LSPR is confined to a small volume, which renders the formation a number of hot spots that enhance the otherwise weak Raman cross section. The LSPR can be easily tuned to match the Raman laser excitation wavelength to achieve the optimal SERS enhancement. Au nanostars stand out among nanoparticles of various shapes for SERS enhancement (such as gold nanosphere, gold nanorod, and nanocube) because of two properties ${ }^{14}$. First, the optical properties of Au nanostar results from the combination of two resonance modes, one at the $\mathrm{Au}$ core and the other at the protruding tips ${ }^{119}$, allowing broad spectral coverage. Second, the sharp tips offer highly confined and therefore highly enhanced electromagnetic fields, significantly boosting the SERS signal.

Despite the excellent electromagnetic properties, local EM field enhancement is limited for a single Au nanostars. To further enhance the local EM field, one way is to take advantage of the LSPR coupling effect among multiple Au nanostars. For example, a Au nanostar dimer system was constructed and tested for mercury ion detection ${ }^{120}$. Even so, coupling nanoparticles still has negative side effects such as a high background due to aggregation and difficulty in separating dimers from single nanoparticles without any analyte present. An alternative is to instead 
hybridize the plasmonic nanoparticle with a plasmonic substrate. The plasmonic substrate can not only help enhance the local EM field, but also provides a way to avoid nanoparticle aggregations and allow separation in the presence of the analyte.

Au nanohole arrays are therefore a high potential plasmonic substrate since it supports both SPP and LSPR modes which can hybridize with the nanoparticle plasmon. As has been revealed in Chapter Three, both resonance modes can be broadly tuned by simply changing the hole size, periodicity, and film thickness to explore the optimum SERS enhancement. It was found that the highest SERS signal was obtained for a periodicity of $600 \mathrm{~nm}$, a hole diameter of $420 \mathrm{~nm}$, and a film thickness of $50 \mathrm{~nm}$, providing an excellent starting point for a $\mathrm{Au}$ nanostar/Au nanohole array coupled SERS sensor for silver ion detection.

Therefore, in this section the favorable properties of the Au nanostar and Au nanohole arrays will be combined to build a SERS sensor for the detection of $\mathrm{Ag}^{+}$. The sensor is assembled as follow. The Raman molecule MGITC is first electrostatically adsorbed on the surface of Au nanostar with a layer of $\mathrm{SiO}_{2}$ resulting in $\mathrm{Au}$ nanostar@MGITC@ $\mathrm{SiO}_{2}$ sandwich nanostructures. The $\mathrm{Au}$ nanostar@MGITC@ $\mathrm{SiO}_{2}$ sandwich nanostructure and Au nanohole array both display LSPR peaks very close to the Raman excitation wavelength of $785 \mathrm{~nm}$, ensuring that the maximum Raman response can be achieved. To allow $\mathrm{Ag}^{+}$detection, $\mathrm{Au}$ nanostar@MGITC@ $\mathrm{SiO}_{2}$ sandwich and Au nanohole array were functionalized with complementary ssDNA sequences except for the three deliberately designed $\mathrm{C}-\mathrm{C}$ matches. In the presence of $\mathrm{Ag}^{+}, \mathrm{Au}$ nanostar@MGITC@SiO 2 sandwich nanoparticles are captured by the Au nanohole array through hybridization of the two complementary ssDNA sequences and the formation of $\mathrm{C}-\mathrm{Ag}^{+}-\mathrm{C}^{121}$. This design strategy brings the LSPR of the nanostar into close proximity of the nanohole array, allowing coupling of the local EM fields, and yielding a limit of detection as low as $170 \mathrm{fM}$ in 
buffer solution. The sensor design outlined is highly promising in detecting trace amount of elements in environmental and biomedical settings.

\subsection{Experimental section}

\subsubsection{Chemicals and Reagents}

DNA sequences of 5'- /5AmMC6/CT CCC CAT A -3' and 5'- /5AmMC6/TA TCC CCA G -3' were synthesized by Integrated DNA Technologies, Inc. Malachite green isothiocyanate (MGITC) was purchased from Molecular Probes, Inc (IDT, Coralville, IA). Chloroauric acid trihydrate $\left(\mathrm{HAuCl}_{4} \cdot 3 \mathrm{H}_{2} \mathrm{O}\right)$, trisodium citrate dehydrate $\left(\mathrm{Na}_{3} \mathrm{C}_{6} \mathrm{H}_{5} \mathrm{O}_{7} \cdot 2 \mathrm{H}_{2} \mathrm{O}\right.$, ACS, 90.0+\%), sodium hydroxide, silver nitrate $\left(\mathrm{AgNO}_{3}\right.$, Premion, 99.995\%), $\mathrm{Na}_{2} \mathrm{HPO}_{4}(99.0 \%), \mathrm{NaH}_{2} \mathrm{PO}_{4}$ (99.0\%), Calcium nitrate tetrahydrate $\left(\mathrm{Ca}\left(\mathrm{NO}_{3}\right)_{2}, 99.0 \%\right)$, Iron (III) nitrate nonahydrate

$\left(\mathrm{Fe}\left(\mathrm{NO}_{3}\right)_{3}, 98+\%\right)$, Copper (II) nitrate hemi (pentahydrate) $\left(\mathrm{Cu}\left(\mathrm{NO}_{3}\right)_{2}\right)$, Yttrium (III) nitrate

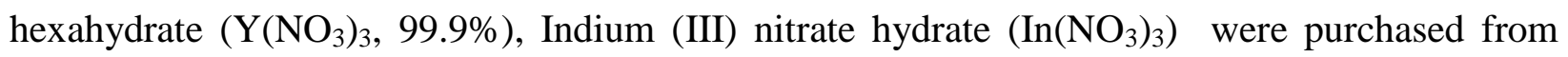
Alfa Aesar. Sodium hydrobromide $\left(\mathrm{NaBH}_{4}\right)$, poly (vinylpyrrolidone) (PVP, 10000), N, Ndimethylformamide (DMF), 11-mercaptoundecanoic acid (MUA), 11-mercapto-1-undecanol (MU), N-hydroxysuccinimide (NHS), 1-ethyl-3-(3-dimethylaminopropyl)-carbodiimide (EDC), Cobalt (II) nitrate hexahydrate $\left(\mathrm{Co}\left(\mathrm{NO}_{3}\right)_{2}, 98+\%\right)$, Chromium (III) nitrate nonahydrate $\left(\mathrm{Cr}\left(\mathrm{NO}_{3}\right)_{3}\right)$ were purchased from Sigma-Aldrich (St. Louis, MO). Nickel Nitrate 6-Hydrate $\left(\mathrm{Ni}\left(\mathrm{NO}_{3}\right)_{2}\right)$ was purchased from ScholAR Chemistry. Aluminium nitrate nonahydrate $\left(\mathrm{Al}\left(\mathrm{NO}_{3}\right)_{3}\right.$, 99+\%) was from ACROS. Zinc nitrate hexahydrate, min $\left(\mathrm{Zn}\left(\mathrm{NO}_{3}\right)_{2}, 98 \%\right)$ came from Strem Chemicals. 3-triethoxysilylpropyl succinic anhydride (TEPSA) was purchased from Gelest Inc. Deionized (D.I.) water was produced by Milli-Q Millipore system (18.2 M $\Omega \mathrm{cm}$, Millipore Corp., 
Billerica, MA) and was used in the whole experiment process for washing or reactions. All chemicals were directly obtained from commercial vendors and used without further purification.

\subsubsection{Apparatus}

A JEOL JSM-7600F scanning electron microscope (SEM) and a JEOL JEM-2100F transmission electron microscope (TEM) were used to image gold nanoparticles. UV-Vis spectra were obtained by a Shimadzu UV-2550 spectrometer with a wavelength range of $200 \sim 900 \mathrm{~nm}$. Raman spectra were collected by a portable Raman spectrometer (Inspector Series, DeltaNu) with an excitation wavelength of $785 \mathrm{~nm}$. An Oxygen Plasma Asher (March PX-250 Plasma Asher) was used to remove the organic groups bonded to the Au film substrates.

\subsubsection{Preparation of Au nanostar@MGITC@SiO2 sandwiched nanoparticles}

Gold star nanoparticles were prepared as reported previously ${ }^{122}$. A $\mathrm{HAuCl}_{4} \cdot 3 \mathrm{H}_{2} \mathrm{O}$ aqueous solution $(1 \mathrm{~mL}, 1 \mathrm{wt} \%)$ was first diluted by water $(90 \mathrm{~mL})$, followed by the injection of sodium citrate $(2 \mathrm{~mL}, 38.8 \mathrm{mM})$. Freshly prepared $\mathrm{NaBH}_{4}$ solution was then slowly added. The mixed solution was stirred overnight to form the seed solution. Subsequently, PVP (10 mM) was dissolved into $50 \mathrm{mM}$ of the above prepared gold seed solution and kept stirred for 24 hours. After that, $82 \mu \mathrm{L}$ of $50 \mathrm{mM} \mathrm{HAuCl}_{4}$ aqueous solution was mixed with $15 \mathrm{~mL}$ of $10 \mathrm{mM}$ PVP in DMF, followed by the rapid addition of $43 \mu \mathrm{L}$ PVP-coated gold seed solution. The reaction lasted about 13 hours. The as-prepared gold nanostar solution was washed by absolute ethanol and D.I. water three times, respectively, and re-dispersed into D.I. water for further use (Figure 4.1). 

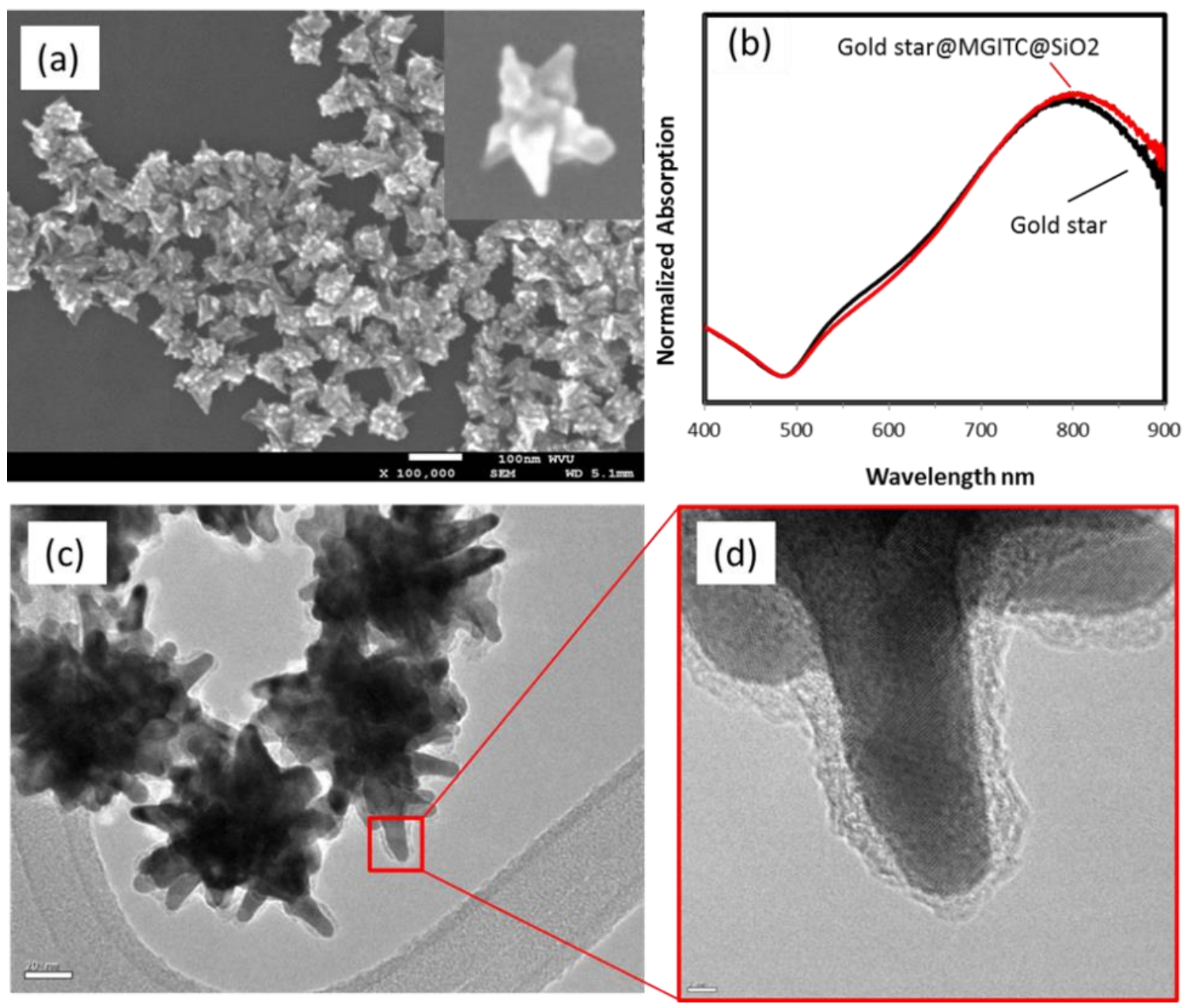

Figure 4.1 (a) SEM image of Au nanostar; (b) UV-Vis absorption spectra of Au nanostar and Au nanostar@MGITC@SiO2; (c) and (d) SEM images of Au nanostar@MGITC@SiO2.

To make sandwiched nanoparticles, $15 \mathrm{uL}$ MGITC was first added to $1 \mathrm{~mL}$ Au nanostar solution (optical density was adjusted to be around 2.5), which was followed by the injection of $200 \mathrm{uL}$ TEOS (1\%). The reaction was allowed to last $30 \mathrm{~min}$. After being kept standing for 2 days, the above solution was washed by ethanol and dispersed into D. I. water for further use (Figure 4.1). If not specified otherwise, all reactions were conducted at room temperature. 


\subsubsection{Preparation of Au nanohole array}

$\mathrm{Au}$ nanohole arrays were fabricated on quartz slides in a cleanroom using nanosphere lithography $(\mathrm{NSL})^{10}$, as shown in Figure 4.2. The quartz slides were cleaned by immersion in an acid piranha bath at $90{ }^{\circ} \mathrm{C}$ for two hours and were then sonicated in acetone, methanol and D. I. water, respectively. A monolayer of polystyrene (PS) microspheres (600 nm in diameter) was next coated on the quartz slides. After drying under ambient conditions, the monolayer-coated quartz slides were placed under oxygen plasma etching, followed by e-beam evaporator deposition of a gold layer. $5 \mathrm{~nm}$ Ti was used as an adhesion layer. Finally, the Au nanohole array was obtained by removing the PS template using sonication in methanol.

(a) Substrate Cleaning quartz

(b) Nanosphere Patterning

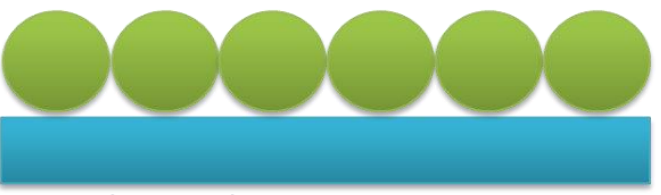

(c) Nanosphere Etching

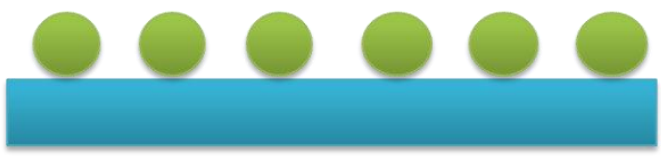

(d) Ti Layer Deposition

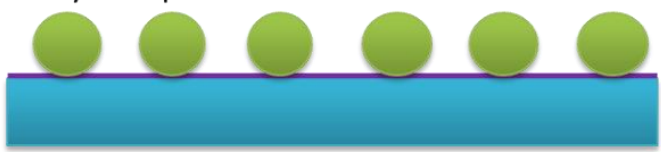

(e) Au Layer Deposition

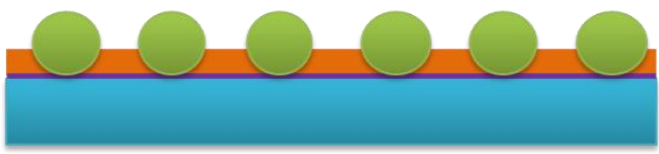

(f) Nanophere Removal



(g)
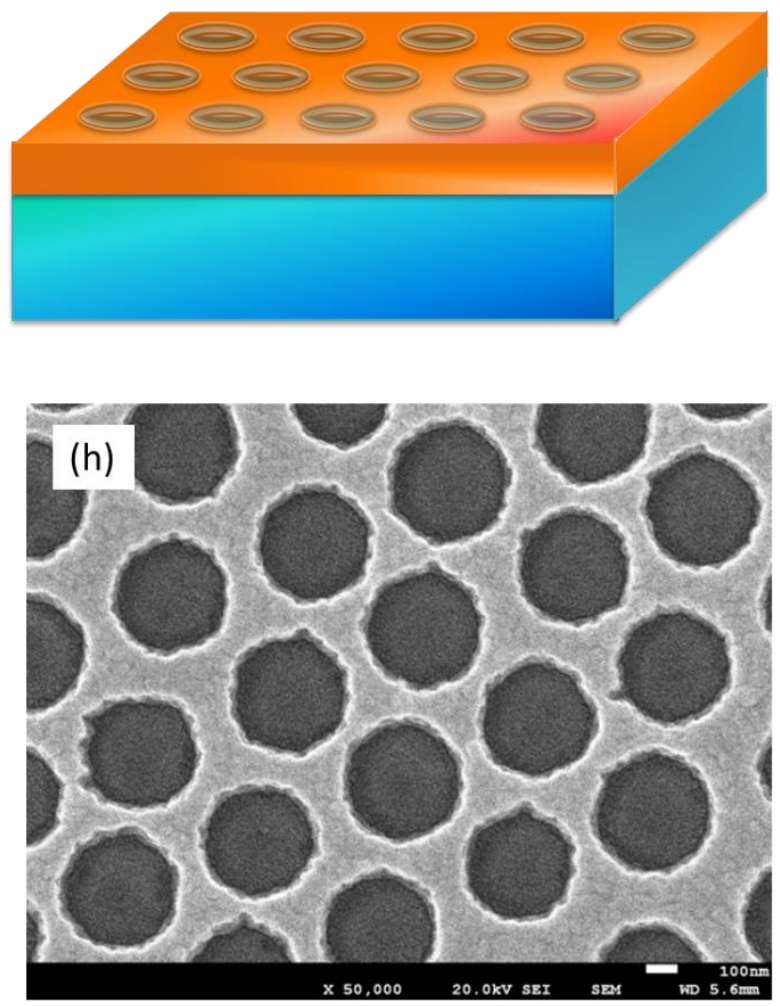
Figure 4.2 (a) to (g) Protocol for Au nanohole arrays fabrications; (h) SEM image of fabricated Au nanohole arrays with a periodicity of $600 \mathrm{~nm}$, a hole diameter of $420 \mathrm{~nm}$, film thickness of $50 \mathrm{~nm}$.

\subsubsection{DNA Functionalization of Au Nanostar@MGITC@SiO ${ }_{2}^{15}$}

Au nanostar@MGITC@ $\mathrm{SiO}_{2}$ was first diluted to $1.5 \mathrm{~mL}$. Then $20 \mathrm{uL}$ TEPSA was added. The mixed aqueous solution was incubated overnight to achieve $\mathrm{COOH}$-terminated $\mathrm{Au}$ nanostar@MGITC@SiO 2 . After washing with ethanol, the precipitate was dissolved into $2 \mathrm{~mL}$ solution containing $50 \mathrm{mM}$ NHS and $200 \mathrm{mM}$ EDC. After incubation for $4 \mathrm{~h}$, the COOH group was activated. Then, $50 \mathrm{uL}$ DNA oligo solution $(20 \mathrm{uM})$ of $5^{\prime}-\mathrm{NH}_{2}-\left(\mathrm{CH}_{3}\right)_{6}-\mathrm{CT}$ CCC CAT A -3' was added. After overnight incubation, the solution was washed with PBS buffer solution (10 $\mathrm{mM}$ MOPS and $30 \mathrm{mM} \mathrm{NaNO}$, $\mathrm{pH}$ 7.0) to get the aptamer-functionalized gold nanoparticles, which were dissolved into PBS buffer solution (10 mM MOPS and $30 \mathrm{mM} \mathrm{NaNO}$, pH 7.0) for further use.

\subsubsection{Functionalization of Au Nanohole array/Au film}

The Au nanohole array substrate was first cleaned by successive sonication in acetone, methanol, and D.I. water for 10 min at each step. After being dried using nitrogen gas, the nanohole arrays were cleaned by an Oxygen Plasma Asher at $300 \mathrm{~W}$ for 2 min. The cleaned Au nanohole array substrates were immersed into the aqueous solution containing $100 \mathrm{mM}$ MUA and $100 \mathrm{mM}$ MU overnight. The resulting MUA/MU functionalized substrates were washed by ethanol and D.I. water and were incubated into $50 \mathrm{mM}$ NHS and $200 \mathrm{mM}$ EDC to get activated. Afterwards, 50 uL DNA oligo solution $(20 \mathrm{uM})$ of $5^{\prime}-\mathrm{NH}_{2}-\left(\mathrm{CH}_{3}\right)_{6}$-TA TCC CCA G -3' was added. After incubation overnight, the substrates were successively washed with PBS buffer solution (10 mM MOPS and $30 \mathrm{mM} \mathrm{NaNO}$, pH 7.0) to remove the unattached DNA oligo. 
The Au film went through the same procedure for functionalization with ssDNA as the $\mathrm{Au}$ nanohole array.

\subsubsection{Preparation of Metal Ions Solutions}

$\mathrm{Ag}^{+}$aqueous solution with various concentrations of $1 \mathrm{pM}, 2 \mathrm{pM}, 5 \mathrm{pM}, 10 \mathrm{pM}, 20 \mathrm{pM}, 50 \mathrm{pM}$, $100 \mathrm{pM}, 200 \mathrm{pM}, 500 \mathrm{pM}, 1 \mathrm{nM}, 2 \mathrm{nM}, 5 \mathrm{nM}, 10 \mathrm{nM}, 20 \mathrm{nM}$, and $50 \mathrm{nM}$ was prepared in PBS buffer solution (10 mM MOPS and $\left.30 \mathrm{mM} \mathrm{NaNO}_{3}, \mathrm{pH}=7.0\right)$. $50 \mathrm{nM}$ metal ion solutions of $\mathrm{Ca}^{2+}$, $\mathrm{Ni}^{2+}, \mathrm{Co}^{2+}, \mathrm{Al}^{3+}, \mathrm{Fe}^{3+}, \mathrm{Cu}^{2+}, \mathrm{Zn}^{2+}, \mathrm{Y}^{3+}, \mathrm{In}^{3+}, \mathrm{Cr}^{3+}$, and a mixture of the above metal ions with $\mathrm{Ag}^{+}$ were also prepared in PBS buffer solution (10 $\mathrm{mM}$ MOPS and $30 \mathrm{mM} \mathrm{NaNO}$, $\mathrm{pH}=7.0$ ) to test the selectivity and anti-interference capability of the biosensor.

\subsection{8 $\mathrm{Ag}^{+}$Detection with SERS Sensor}

35 uL DNA-modified Au nanostar@MGITC@ $\mathrm{SiO}_{2}$ sandwich nanoparticle solution was first dropped on the DNA-functionalized Au nanohole array (Au film) substrate, followed by another drop (35 uL) of $\mathrm{Ag}^{+}$solution with different concentrations. After a 16 min incubation, the substrates were washed with PBS buffer (10 $\mathrm{mM}$ MOPS and $30 \mathrm{mM} \mathrm{NaNO}_{3}, \mathrm{pH}$ 7.0) to remove the unbounded Au nanostar@MGITC@ $\mathrm{SiO}_{2}$ nanoparticles. Nitrogen gas was blown to dry the substrates. For each sample, the Raman spectra were acquired at three different points on the nanohole array with an accumulation time of 2 seconds. The SERS sensor working conditions were first optimized as shown in Figure 4.3. 

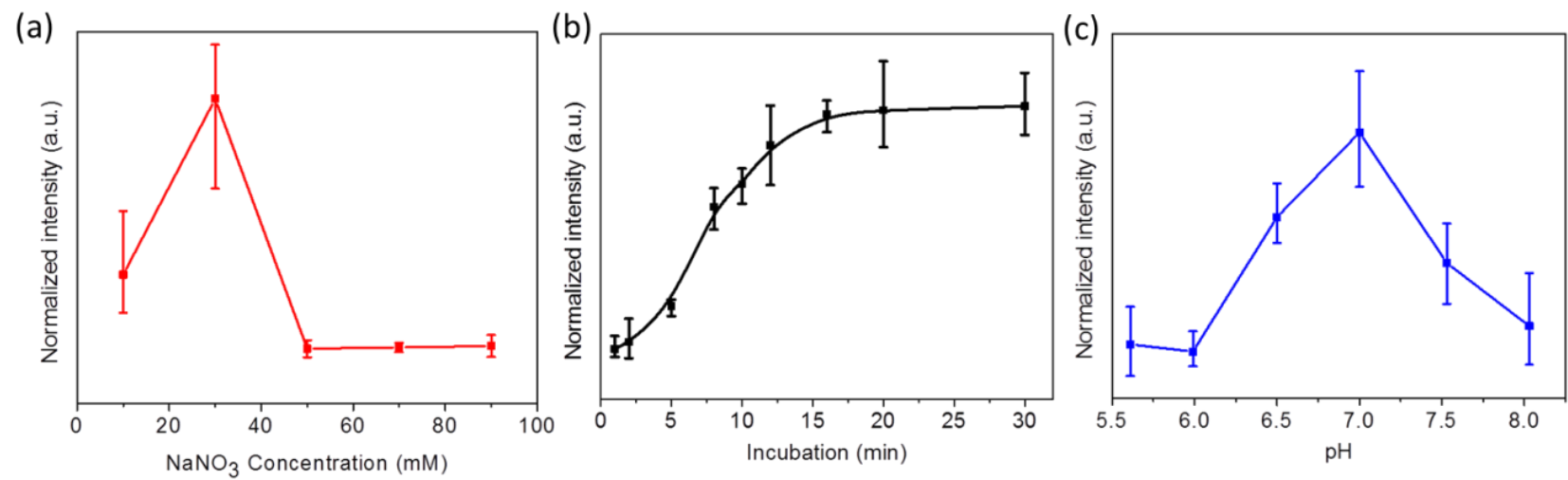

Figure 4.3 SERS sensor working conditions optimization (a) Ionic strength optimization. The optimal ionic strength was found to be $30 \mathrm{mM}$; (b) Incubation time optimization with the optimal value of $16 \mathrm{~min}$; (c) $\mathrm{pH}$ optimization. Neutral $\mathrm{pH}$ is preferred.

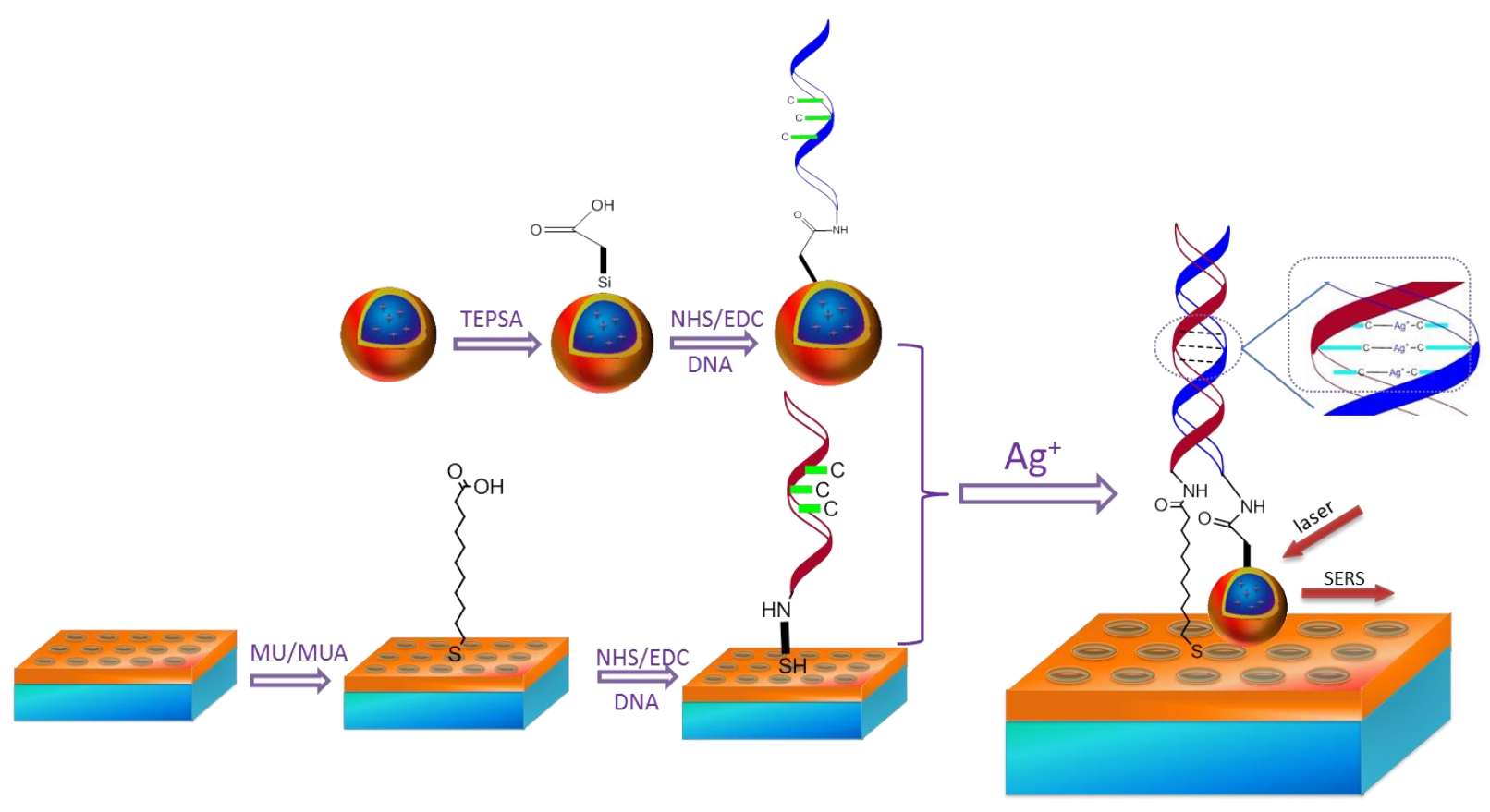


the SERS sensor operation principle.Au nanostar@MGITC@ $\mathrm{SiO}_{2}$ sandwich was functionalized with single-stranded DNA sequence of 5' $-\mathrm{NH}_{2}-\left(\mathrm{CH}_{3}\right)_{6}$-CT CCC CAT A -3'; Au film was functionalized with single-stranded DNA sequence of 5' $-\mathrm{NH}_{2}-\left(\mathrm{CH}_{3}\right)_{6}$-TA TCC CCA G -3'. 


\subsection{Results and discussion}

\subsubsection{Operation Mechanism of SERS Sensor}

The primary idea of the SERS sensor is to use of $\mathrm{Ag}^{+}$as a bridge to bring $\mathrm{Au}$ nanostar@MGITC@ $\mathrm{SiO}_{2}$ to the surface of the $\mathrm{Au}$ nanohole array (Figure 4.4) through the formation of $\mathrm{C}-\mathrm{Ag}^{+}-\mathrm{C}^{121}$. The Au nanohole array then offers the platform on which the detection takes place. Since the Raman molecules are sandwiched into the structure of the $\mathrm{Au}$ nanostar@MGITC@ $\mathrm{SiO}_{2}$, a SERS signal will only exist when $\mathrm{Ag}^{+}$ions are present. The SERS signal therefore allows for the $\mathrm{Ag}^{+}$concentration to be accurately quantified.

\subsubsection{Comparison of SERS Sensor Performance on Au Nanohole Array and Au film}

Both Au nanohole arrays and Au films were used to test the performance of the SERS sensor. The experimental process flow is as follows. First, Au nanostar@MGITC@ $\mathrm{SiO}_{2}$ solution was dropped on the ssDNA-functionalized substrate, which wass immediately followed by another drop of $\mathrm{Ag}^{+}$solution with varying concentration. The hybridization of the two single-stranded DNA sequences took place with the formation of $\mathrm{C}-\mathrm{Ag}^{+}-\mathrm{C}$, and after 16 min of incubation, the amount of Au nanostar@MGITC@SiO2 nanoparticles bonded on the substrate became saturated (Figure 4.3 (b)). The substrate was next rinsed with PBS buffer solution $(\mathrm{pH}=7.0)$ and dried using nitrogen gas. Finally, the Raman spectra were acquired by illuminating the substrate.

The SERS signal was found to be proportional to the $\mathrm{Ag}^{+}$concentration for low values (Figure 5(a)). Above $10 \mathrm{nM}$, the SERS signal got saturated as a result of the fully occupied bonding sites. For the linear region, a calibration curve was obtained by plotting the peak at $1174 \mathrm{~cm}^{-1}$ with respect to the logarithmic concentration of $\mathrm{Ag}^{+}$. The linear range was determined to be from 2 pM to $10 \mathrm{nM}$ with the fitting equation as $\mathrm{y}=1400 \mathrm{x}+110, \mathrm{R}^{2}=99 \%$ for Au nanohole array and 
$y=370 x+330, R^{2}=98 \%$ for Au film, where $y$ is the SERS intensity at $1174 \mathrm{~cm}^{-1}, x$ is the logarithmic concentration of $\mathrm{Ag}^{+}$. The slope of the fitting equation indicates the sensitivity of the SERS sensor, meaning the sensor built on the Au nanohole array is about 3.8 times more sensitive than that built on Au film. The limit of detection (LOD) was estimated based on three times the standard deviation/slope ${ }^{123}$ by the International Union of Pure and Applied Chemistry (IUPAC) standard. The results showed a LOD of $170 \mathrm{fM}$ for Au nanohole array and $700 \mathrm{fM}$ for Au film.

The Au nanohole proved to be a better plasmonic substrate for the SERS sensor. Compared to most conventional fluorescence methods ${ }^{106,107,113}$, the SERS sensor is advantageous in that it reaches a higher sensitivity and a lower LOD. The reason of the better performance of Au nanohole array may be ascribed to the synergistic coupling of the local fields of the Au nanostar and Au nanohole array, of which is impossible in the non-patterned Au film. 



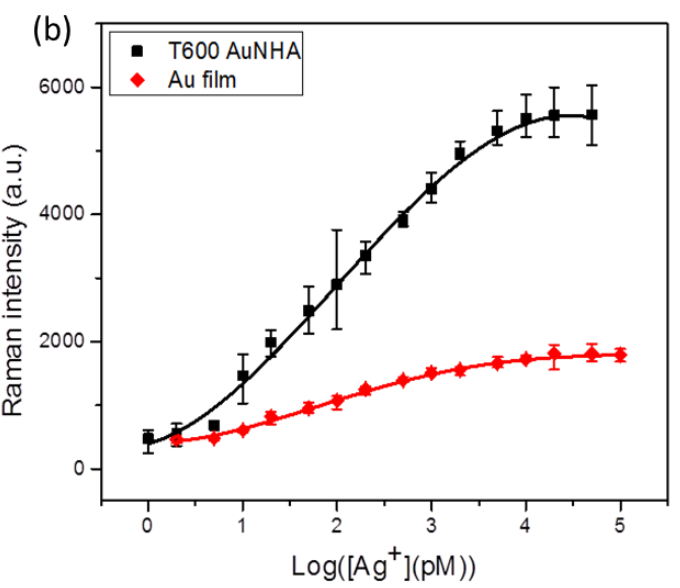

(d)

\begin{tabular}{lcc}
\hline & T600 AuNHA & Au film \\
\hline Detection range & $1 \mathrm{pM} \sim 50 \mathrm{nM}$ & $2 \mathrm{pM} \sim 100 \mathrm{nM}$ \\
Linear range & $2 \mathrm{pM} \sim 10 \mathrm{nM}$ & $5 \mathrm{pM} \sim 10 \mathrm{nM}$ \\
Limit of detection & $170 \mathrm{fM}$ & $700 \mathrm{fM}$ \\
\hline
\end{tabular}

Figure 4.5 (a) SERS spectra of Au nanostar@MGITC@ $\mathrm{SiO}_{2}$ on Au nanohole array in the SERS sensor for the detection of $\mathrm{Ag}^{+}$. (b) Plots of SERS peak intensity at $1174 \mathrm{~cm}^{-1}$ as a function of the logarithmic concentration of $\mathrm{Ag}^{+}$. (c) Calibration of the linear range of (b). (d) Performance of the SERS sensor. The black curve stands for the sensor on Au nanohole array while the red curve stands for that on Au film.

\subsubsection{Selectivity}

A selectivity test was conducted to check the SERS sensor's anti-inference capability. A series of metal ions, $\mathrm{Al}^{3+}, \mathrm{Ca}^{2+}, \mathrm{Cr}^{3+}, \mathrm{Cu}^{2+}, \mathrm{Fe}^{3+}, \mathrm{In}^{3+}, \mathrm{In}^{3+}, \mathrm{Ni}^{2+}, \mathrm{Y}^{3+}, \mathrm{Zn}^{2+}$, plus a mixture of the above ions with $\mathrm{Ag}^{+}$were prepared with a concentration of $50 \mathrm{nM}$ in the buffer solution of $10 \mathrm{mM}$ MOPS and $30 \mathrm{mM} \mathrm{NaNO}$ at $\mathrm{pH}$ 7.0. In Figure 4.6, the test results show $\mathrm{Ag}^{+}$gives a high Raman intensity while the other metal ions only give a limited Raman response due to the unspecific bonding that results from the $\mathrm{C}-\mathrm{C}$ mismatch. 


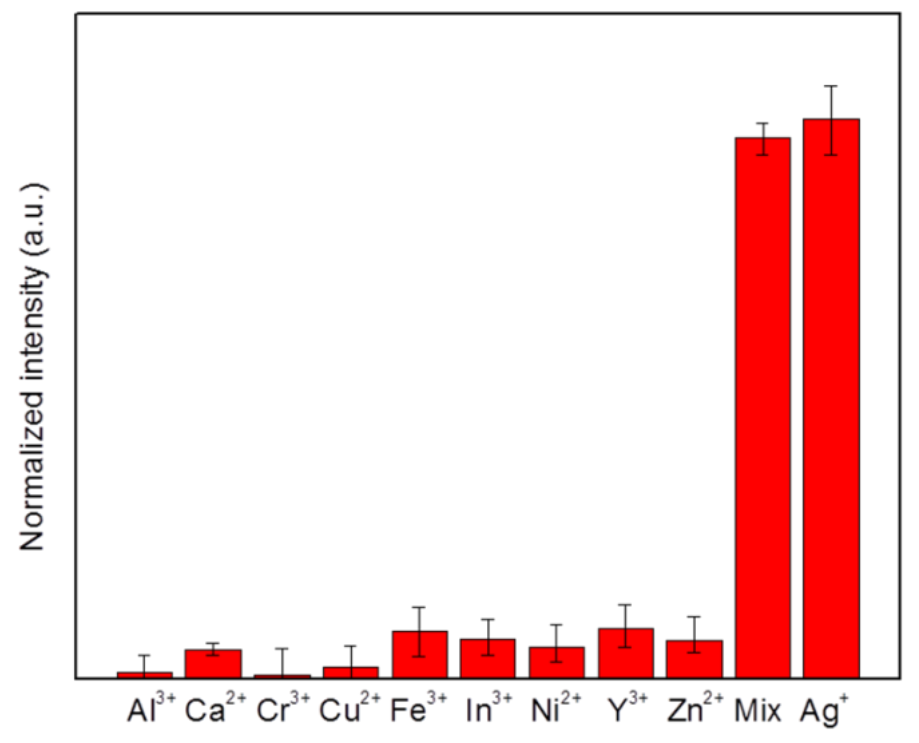

Figure 4.6 Selectivity test of the SERS sensor against metal ions: $\mathrm{Al}^{3+}, \mathrm{Ca}^{2+}, \mathrm{Cr}^{3+}, \mathrm{Cu}^{2+}, \mathrm{Fe}^{3+}, \mathrm{In}^{3+}, \mathrm{In}^{3+}$, $\mathrm{Ni}^{2+}, \mathrm{Y}^{3+}, \mathrm{Zn}^{2+}$, a mixture of the above ions with $\mathrm{Ag}^{+}$, and $\mathrm{Ag}^{+}$. All of the solution has a concentration of $50 \mathrm{nM}$ in the buffer solution of $10 \mathrm{mM}$ MOPS and $30 \mathrm{mM} \mathrm{NaNO}_{3}$ at $\mathrm{pH}$ 7.0.

\subsubsection{SERS Enhancement by Au Nanohole array}

Au nanohole arrays provide a plasmonic platform with a lower LOD, higher slope, and higher selectivity toward silver ions. The increase in performance compared to a Au film is attributed to the coupling between the Au nanostar and Au nanohole array. FDTD simulations were used to clarify that the local electromagnetic field led to the measured increases in performance relative to a single nanoparticle and a bare Au film.

As the Au nanostar could be found at any location on the Au nanohole array, various scenarios were considered in the FDTD simulation. Figure 4.7 (a) is an SEM image of an Au nanohole array chip with a periodicity of $600 \mathrm{~nm}$, a film thickness of $50 \mathrm{~nm}$, and a hole diameter of 420 $\mathrm{nm}$. Two areas were extracted from the image for the FDTD simulation. For the red dashed rectangle, shown in Figure 4.7 (c), Au nanostars were modelled to be located at point A, B, C, 
and D. Point A being in the middle of the gap between two nearest holes; point B being located at the right edge of a nanohole; point $\mathrm{C}$ being at the center of the gap between three closest holes; point D being at the lower edge of a hole. For the green dashed rectangle, shown in Figure 4.7 (b), the Au nanostar was modelled to be located at the center of the nanohole, to check the small chance that the Au nanostar could sit inside the hole despite this area not being functionalized with ssDNA. The two kinds simulation cells were created to ensure the most probable locations of Au nanostar on $\mathrm{Au}$ nanohole array had been taken into account. For all the simulations, a monochromatic incident light with a wavelength of $785 \mathrm{~nm}$ was used to match the laser used in real experiments.

(a)

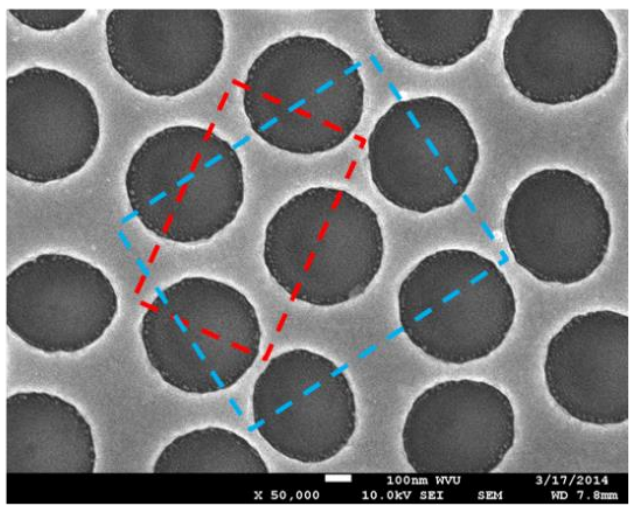

(c)

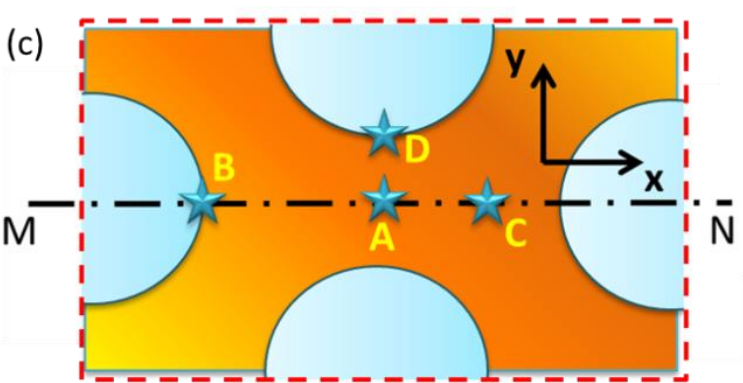

(b)

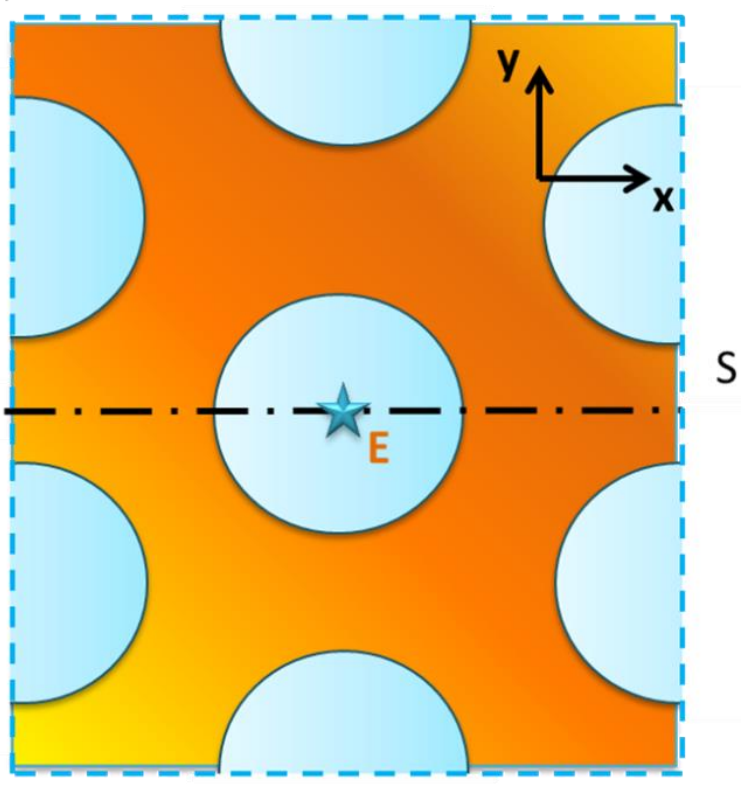

Figure 4.7 (a) SEM image of Au nanohole array with a periodicity of $600 \mathrm{~nm}$; the dashed red and green rectangles correspond to (b) and (c) in FDTD simulations. (b) One kind of FDTD simulation cell: point E shows Au nanostar is put at the center of a nanohole (c) The other kind of FDTD simulation cell with Au nanostar put at four different locations: point $\mathrm{A}$ is in the middle of the gap between two closest nanoholes; 
point $\mathrm{B}$ is at the right edge of a nanohole; point $\mathrm{C}$ is in the center of the gap between three closest nanoholes; point $\mathrm{D}$ is at the lower edge of a nanohole.

Figure 4.8 (a), (d) and Figure 4.9 (a) reveal that the EM field of the Au nanohole array was localized at the rim of the holes when the LSPR was excited. Therefore, for Au nanostars to have a strong coupling with the LSPR mode of the Au nanohole array, they must be as close to the rim of the holes as possible. Of all the simulated positions, point $\mathrm{B}$ and $\mathrm{D}$ were the most probable position for the coupling to happen. Figure 4.8 (b) reveals that under x polarization, an $\mathrm{Au}$ nanostar sitting at point B (rim of the hole) strongly interacted with the LSPR mode of the Au nanohole array, resulting in a SERS enhancement factor of $7.8 \times 10^{6}$. Under y polarization, point D had the same coupling with the LSPR mode with an enhancement factor of $2.3 \times 10^{6}$. The other positions represented by point A, C, and E, as shown in Figure 4.8 and Figure 4.9, did not support strong coupling between Au nanostar because they were far from the short decay length of the LSPR field. Table 1 and Table 2 list all the simulated SERS enhancement factors under the different placement positions. In contrast, Au nanostar had a relatively weak coupling with $\mathrm{Au}$ film. The enhancement factor was calculated to be round $2.4 \times 10^{4}$, explaining the difference in performance. 

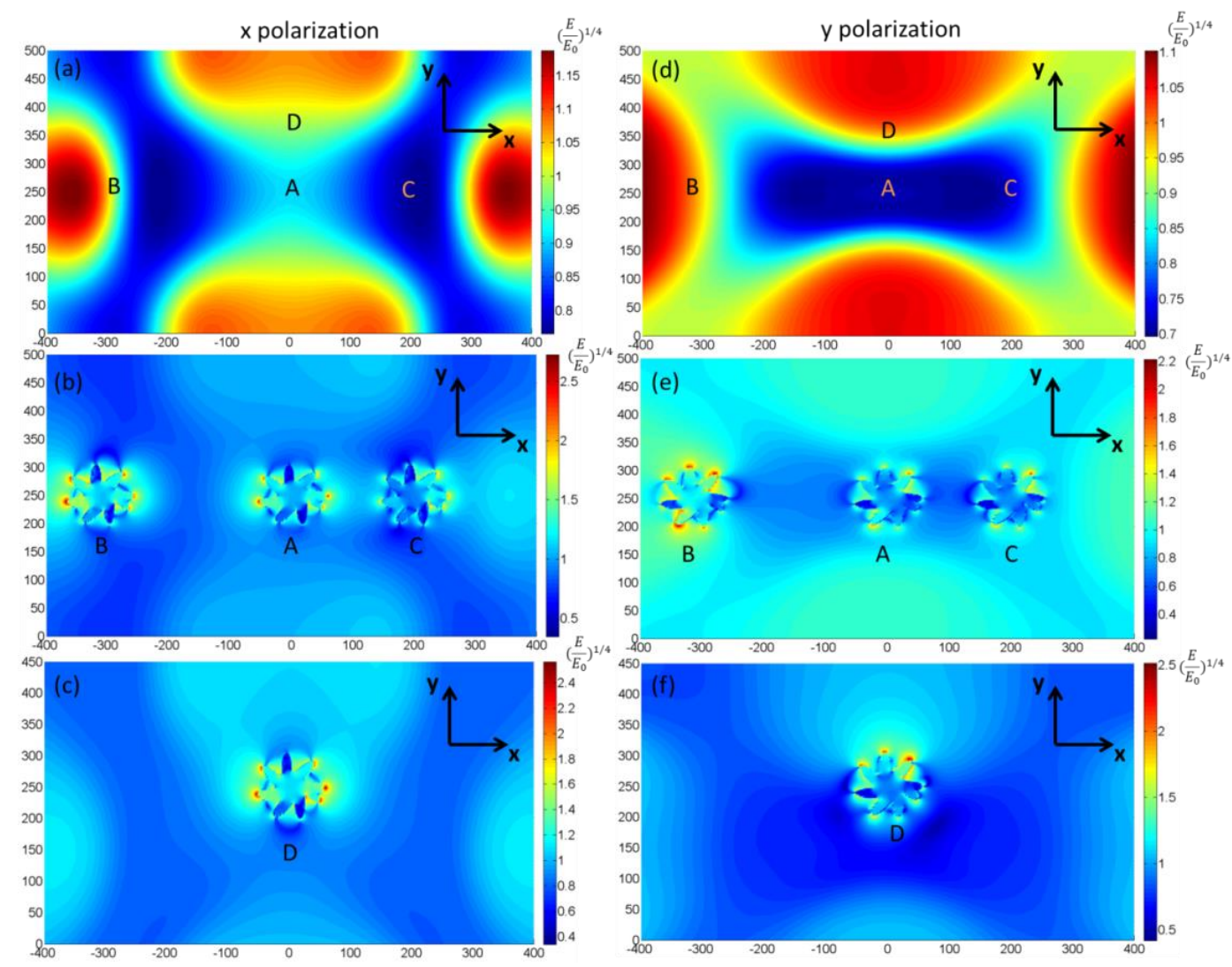

Figure 4.8 FDTD simulated electromagnetic field distributions with $785 \mathrm{~nm}$ incident light. (a) and (d) show the electromagnetic fields on Au nanohole arrays with a periodicity of $600 \mathrm{~nm}$ and hole diameter of $420 \mathrm{~nm}$ using $\mathrm{x}$ - and y-polarized incident light, respectively; (b) to (c) and (e) to (f) show the field enhancement of Au nanostars located at different places on Au nanohole array with x- and y-polarized light, respectively. 


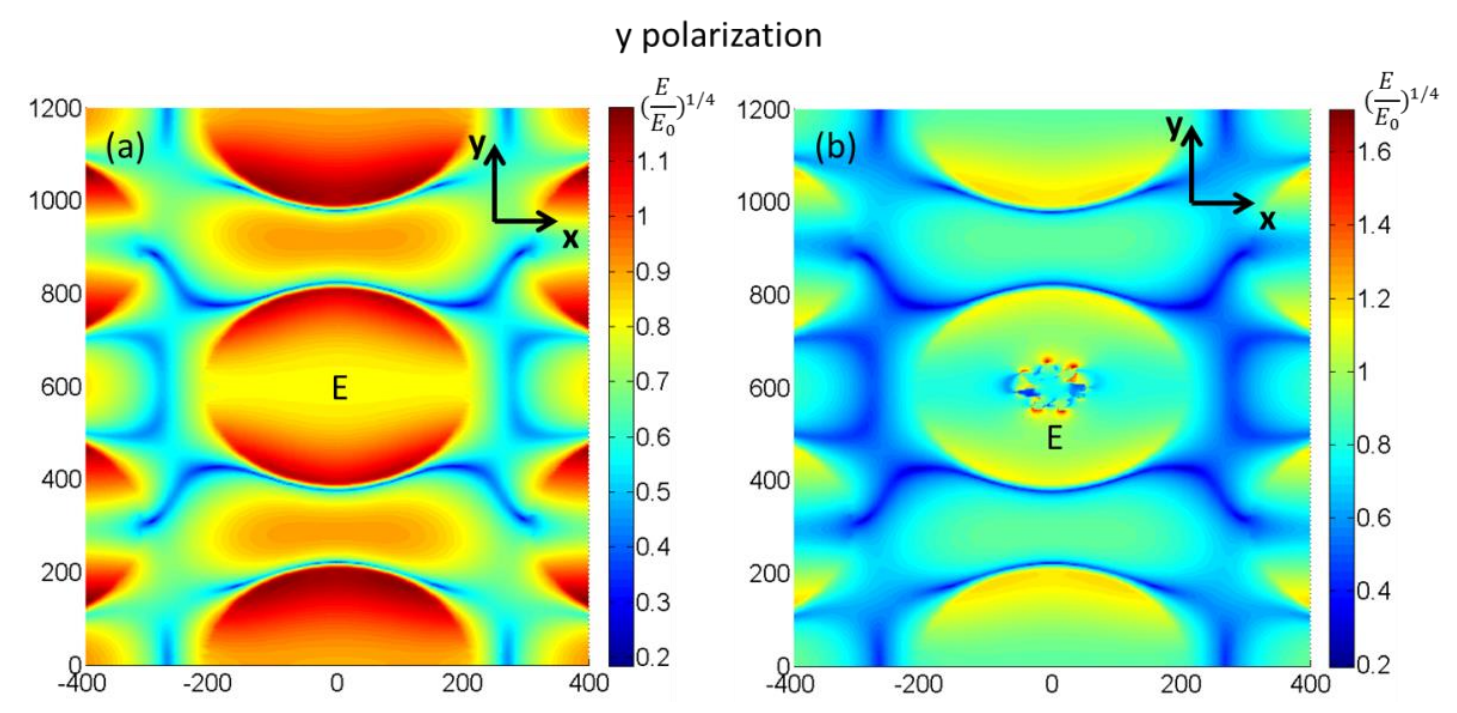

Figure 4.9 FDTD simulated electromagnetic field distributions with a y-polarized $785 \mathrm{~nm}$ incident light.

(a) the field distribution on Au nanohole array with a periodicity of $600 \mathrm{~nm}$ and diameter of $420 \mathrm{~nm}$; (b) the field enhancement of Au nanostar at the center of nanohole.

\begin{tabular}{ccccccc}
\hline (E/E0)^4 & \multicolumn{3}{c}{ M-N cross-section } & D & R-S cross-section \\
\cline { 2 - 4 } & A & B & C & & E \\
Px & $8.7 E+03$ & $1.2 \mathrm{E}+06$ & $4.6 \mathrm{E}+05$ & & N/A & $4.2 \mathrm{E}+02$ \\
\hline $\mathrm{Py}$ & $4.5 \mathrm{E}+04$ & $6.2 \mathrm{E}+05$ & $1.5 \mathrm{E}+04$ & N/A & $1.1 \mathrm{E}+04$ \\
\hline
\end{tabular}

Table 4.1 SERS enhancement factors of Au nanostars at different locations on Au nanohole array. The SERS enhancement factors in the table were from the M-N and R-S cross-sections. The M-N corsssection was shown in Figure 4.10 (c); R-S cross-section was shown in Figure 4.11 (d).

\begin{tabular}{cccccc}
\hline (E/E0)^4 $^{\wedge}$ & \multicolumn{5}{c}{ X-Y cross-section } \\
\cline { 2 - 6 } & A & B & C & D & E \\
\hline $\mathrm{Px}$ & $1.4 \mathrm{E}+06$ & $7.8 \mathrm{E}+06$ & $8.0 \mathrm{E}+05$ & $3.3 \mathrm{E}+06$ & $7.6 \mathrm{E}+03$ \\
\hline $\mathrm{Py}$ & $9.0 \mathrm{E}+03$ & $3.5 \mathrm{E}+05$ & $4.0 \mathrm{E}+04$ & $2.3 \mathrm{E}+06$ & $5.2 \mathrm{E}+03$ \\
\hline
\end{tabular}

Table 4.2 SERS enhancement factors of Au nanostars at different locations on Au nanohole array. The SERS enhancement factors in the table were from the $\mathrm{X}$-Y cross-sections. 
The excellent performance of the SERS sensor built with Au nanostar on Au nanohole array could thus be understood as a result of the strong coupling between the Au nanostar and the LSPR mode of the Au nanohole array. While different positions on the Au nanohole array supported different levels of coupling, the rim area provided the highest enhancement factor while the gap area offered generally lower enhancement factors, still producing an average enhancement larger than nanostars on the Au film.

\subsection{Conclusion}

In summary, we have successfully developed a SERS sensor for silver ions detection based on the strong coupling between $\mathrm{Au}$ nanostar and $\mathrm{Au}$ nanohole array. The presence of $\mathrm{Ag}^{+}$leads to the formation of $\mathrm{C}-\mathrm{Ag}^{+}-\mathrm{C}$ pairs, resulting in the hierarchical nanostructure of $\mathrm{Au}$ nanostar@MGITC@ $\mathrm{SiO}_{2}$ via $\quad$ DNA $\quad$ hybridizations. The Au@MGITC@ $\mathrm{SiO}_{2}$ sandwich nanoparticles are selectively binded to create Raman signal intensity proportional to the logarithmic concentration of $\mathrm{Ag}^{+}$. This approach provides a facile way for metal ion detection with high sensitivity and selectivity. It is expected that the hierarchical nanostructure is promising for integration into microfluidic system for on-site sensing applications. 


\section{Chapter 5: Conclusions and Recommendations on Future Work}

\subsection{Conclusions}

In this thesis, a systematic study of the optical properties of Au nanoarrays was conducted, revealing a strong correlation between the Raman performance and the local electric field enhancement of the order, disorder, and reflectance. On the basis of this understanding of the Raman response for Au nanoarrays with different parameters, an optimized $\mathrm{Au}$ nanohole array with a periodicity of $600 \mathrm{~nm}$, a hole diameter of $420 \mathrm{~nm}$, and a film thickness of $50 \mathrm{~nm}$, was employed to build an SERS sensor for silver ion detection. The strong LSPR coupling between Au nanostar and Au nanohole array was utilized to create a high sensitivity and very low limit of detection sensor.

More detailed conclusions are as follows:

(1) Au nanotriangle arrays behave like nanoparticles which are dominated by the LSPR resonance mode; in contrast, in addition to the LSPR resonance mode, Au nanohole arrays can also support SPP modes;

(2) The transition zone between the Au nanotriangle array and the Au nanohole array exists when the film networks of Au nanohole arrays are seamlessly formed. Specific to Au nanoarrays with a periodicity of $500 \mathrm{~nm}$, the transition zone takes place when the hole size lies in the range of $440 \mathrm{~nm} \sim 410 \mathrm{~nm}$; to Au nanoarrays with a periodicity of $600 \mathrm{~nm}$, it takes place when the hole size is between $540 \mathrm{~nm} \sim 510 \mathrm{~nm}$;

(3) The refractive index sensitivity of Au nanotriangle arrays is small but is subject to a steady increase at the transition zone since the electromagnetic field changes drastically. 
The LSPR peak position also shifts in this range as the resonance goes from metal nanoparticle to a void in the metal film;

(4) For Au nanohole arrays, the LSPR resonance mode is independent of the incident angle but the SPP resonance mode is angle-resolved, which is imposed by the momentum conservation condition;

(5) The resonance modes of Au nanoarrays show a dependence on the hole diameter and periodicity. A decrease of the hole diameter or an increase of the periodicity leads to a red shift of the resonance modes. Au film thicker than $40 \mathrm{~nm}$ do not influence the resonance modes but below $40 \mathrm{~nm}$, the resonance modes red shift with a further decrease of the film thickness due to the interactions of the SPP modes on both sides of the Au nanoarray;

(6) The Raman response of Au nanoarrays was found to be a synergistic effect of the electric field enhancement of both defect-containing and defect-free nanoarray and the reflectance;

(7) When the LSPR resonance mode overlaps with the Raman excitation wavelength, there will be an optimal Raman performance, which was demonstrated by Au nanohole arrays with a periodicity of $600 \mathrm{~nm}$, a hole diameter of $420 \mathrm{~nm}$, a film of $50 \mathrm{~nm}$, and a LSPR wavelength of around $780 \mathrm{~nm}$;

(8) A SERS sensor built on the optimized Au nanohole array exhibited improved sensitivity compared to a Au film, a lower limit of detection, and a strong selectivity towards silver ions. The improved performance is due to the strong coupling between the Au nanostar and Au nanohole array. 


\subsection{Recommendations on Future Work}

Study of the optical properties of Au nanoarrays provides us with a guideline in selecting the right Au nanohole array for building SERS sensors. The demonstration of a SERS sensor to detect silver ions under laboratory conditions shows the potential of the sensor to be implemented in real life applications. Based on the work done in this thesis, the following recommendations are made for future work:

(1) Establish a protocol, an archive, or database regarding the control of the optical properties, especially the Raman response, of Au nanoarrays. This requires an expansion of the Au nanoarrays being studied both experimentally and theoretically by FDTD simulations. It is expected a stronger correlation between the Raman response and the optical properties of Au nanoarrays will be observed;

(2) Assess the capability of the SERS sensor with real samples, like river water and human fluids. A lack of a feasibility test using real substances greatly restrain and highly limits the application of the SERS sensor;

(3) The working mechanism of the SERS sensor is based on $\mathrm{C}-\mathrm{Ag}^{+}-\mathrm{C}$ bonding, which is highly specific. Following this principle, it is necessary and beneficial to explore similar structures and reaction mechanism in order to develop sensors capable of wide range detection applications. 


\section{References}

1. T. W. Ebbesen, H. J. Lezec, H. F. Ghaemi, T. Thio and P. A. Wolff, Nature, 1998, 391, 667-669.

2. S. H. Lee, T. W. Johnson, N. C. Lindquist, H. Im, D. J. Norris and S. H. Oh, Adv Funct Mater, 2012, 22, 4439-4446.

3. L. Martin-Moreno, F. J. Garcia-Vidal, H. J. Lezec, K. M. Pellerin, T. Thio, J. B. Pendry and T. W. Ebbesen, Phys Rev Lett, 2001, 86, 1114-1117.

4. S. H. Chang, S. K. Gray and G. C. Schatz, Opt Express, 2005, 13, 3150-3165.

5. J. Homola, Chem Rev, 2008, 108, 462-493.

6. A. Hessel and A. A. Oliner, Appl Optics, 1965, 4, 1275-1295.

7. R. H. Ritchie, Phys Rev, 1957, 106, 874-881.

8. S. A. Meyer, B. Auguie, E. C. Le Ru and P. G. Etchegoin, J Phys Chem A, 2012, 116, 1000-1007.

9. E. K. Akowuah, T. Gorman and S. Haxha, Opt Express, 2009, 17, 23511-23521.

10. C. L. Haynes and R. P. Van Duyne, J Phys Chem B, 2001, 105, 5599-5611.

11. J. C. Hulteen, D. A. Treichel, M. T. Smith, M. L. Duval, T. R. Jensen and R. P. Van Duyne, J Phys Chem B, 1999, 103, 3854-3863.

12. P. Colson, C. Henrist and R. Cloots, J Nanomater, 2013. 
13. M. Li, J. M. Zhang, S. Suri, L. J. Sooter, D. L. Ma and N. Q. Wu, Anal Chem, 2012, 84, 2837-2842.

14. M. Li, S. K. Cushing, J. M. Zhang, J. Lankford, Z. P. Aguilar, D. L. Ma and N. Q. Wu, Nanotechnology, 2012, 23.

15. M. Li, S. K. Cushing, H. Y. Liang, S. Suri, D. L. Ma and N. Q. Wu, Anal Chem, 2013, 85, 2072-2078.

16. M. Li, S. K. Cushing, J. M. Zhang, S. Suri, R. Evans, W. P. Petros, L. F. Gibson, D. L. Ma, Y. X. Liu and N. Q. Wu, Acs Nano, 2013, 7, 4967-4976.

17. A. V. Zayats and I. I. Smolyaninov, J Opt a-Pure Appl Op, 2003, 5, S16-S50.

18. M. A. Ordal, L. L. Long, R. J. Bell, S. E. Bell, R. R. Bell, R. W. Alexander and C. A. Ward, Appl. Opt., 1983, 22, 1099-1119.

19. L. Wu, P. Bai and E. P. Li, J Opt Soc Am B, 2012, 29, 521-528.

20. A. V. Zayats, I. I. Smolyaninov and A. A. Maradudin, Phys Rep, 2005, 408, 131-314.

21. W. L. Barnes, A. Dereux and T. W. Ebbesen, Nature, 2003, 424, 824-830.

22. R. P. Van Duyne, Science, 2004, 306, 985-986.

23. A. K. Singh and S. C. Sharma, Opt Laser Technol, 2014, 56, 256-262.

24. H.-S. Leong, J. Guo, R. G. Lindquist and Q. H. Liu, J Appl Phys, 2009, 106, 124314. 
25. S. Wu, P. F. Guo, W. X. Huang, S. J. Xiao and Y. Y. Zhu, J Phys Chem C, 2011, 115, 15205-15209.

26. K. L. Kelly, E. Coronado, L. L. Zhao and G. C. Schatz, J Phys Chem B, 2003, 107, 668677.

27. L. P. Du, X. J. Zhang, T. Mei and X. C. Yuan, Opt Express, 2010, 18, 1959-1965.

28. H. A. Bethe, Phys Rev, 1944, 66, 163-182.

29. M. Najiminaini, F. Vasefi, B. Kaminska and J. J. L. Carson, Plasmonics, 2013, 8, 217224.

30. M. Couture, L. S. Live, A. Dhawan and J. F. Masson, Analyst, 2012, 137, 4162-4170.

31. C. Genet and T. W. Ebbesen, Nature, 2007, 445, 39-46.

32. H. W. Gao, J. Henzie and T. W. Odom, Nano Lett, 2006, 6, 2104-2108.

33. H. F. Ghaemi, T. Thio, D. E. Grupp, T. W. Ebbesen and H. J. Lezec, Phys Rev B, 1998, 58, 6779-6782.

34. T. Thio, H. F. Ghaemi, H. J. Lezec, P. A. Wolff and T. W. Ebbesen, J Opt Soc Am B, 1999, 16, 1743-1748.

35. T. Rindzevicius, Y. Alaverdyan, B. Sepulveda, T. Pakizeh, M. Kall, R. Hillenbrand, J. Aizpurua and F. J. G. de Abajo, J Phys Chem C, 2007, 111, 1207-1212.

36. R. Gordon, A. G. Brolo, D. Sinton and K. L. Kavanagh, Laser Photonics Rev, 2010, 4, 311-335. 
37. A. Hessel and A. A. Oliner, Appl. Opt., 1965, 4, 1275-1297.

38. Y. Shen, J. H. Zhou, T. R. Liu, Y. T. Tao, R. B. Jiang, M. X. Liu, G. H. Xiao, J. H. Zhu, Z. K. Zhou, X. H. Wang, C. J. Jin and J. F. Wang, Nat Commun, 2013, 4.2318.

39. M. P. Murray-Methot, M. Ratel and J. F. Masson, J Phys Chem C, 2010, 114, 8268-8275.

40. Y. Ikenoya, M. Susa, J. Shi, Y. Nakamura, A. B. Dahlin and T. Sannomiya, J Phys Chem C, 2013, 117, 6373-6382.

41. M. Couture, Y. Z. Liang, H. P. P. Richard, R. Faid, W. Peng and J. F. Masson, Nanoscale, 2013, 5, 12399-12408.

42. S. K. Gray, J Phys Chem C, 2013, 117, 1983-1994.

43. J. P. Guo and H. S. Leong, Appl Phys Lett, 2012, 101.241115.

44. T. H. Reilly, R. C. Tenent, T. M. Barnes, K. L. Rowlen and J. van de Lagemaat, Acs Nano, 2010, 4, 615-624.

45. J. F. Masson, K. F. Gibson and A. Provencher-Girard, J Phys Chem C, 2010, 114, 2240622412.

46. L. Pang, G. M. Hwang, B. Slutsky and Y. Fainman, Appl Phys Lett, 2007, 91.

47. M. K. Liu, Y. J. Song, Y. B. Zhang, X. H. Wang and C. J. Jin, Plasmonics, 2012, 7, 397410.

48. Y. Shen, X. Chen, Z. J. Dou, N. P. Johnson, Z. K. Zhou, X. H. Wang and C. J. Jin, Nanoscale, 2012, 4, 5576-5580. 
49. A. Bottomley, D. Prezgot and A. Ianoul, Appl Phys a-Mater, 2012, 109, 869-872.

50. A. Bottomley and A. Ianoul, Quantum Dots and Nanostructures: Synthesis, Characterization, and Modeling Xi, 2014, 8996.

51. A. Hajiaboli, M. Kahrizi and V. V. Truong, J Phys D Appl Phys, 2012, 45. 485105.

52. J. Parsons, E. Hendry, C. P. Burrows, B. Auguie, J. R. Sambles and W. L. Barnes, Phys $\operatorname{Rev} B, 2009,79.073412$.

53. K. A. Tetz, R. Rokitski, M. Nezhad and Y. Fainman, Appl Phys Lett, 2005, 86. 111110.

54. $\quad$ L. Pang, K. A. Tetz and Y. Fainman, Appl Phys Lett, 2007, 90.

55. L. S. Live, O. R. Bolduc and J. F. Masson, Anal Chem, 2010, 82, 3780-3787.

56. K. Cheng, S. J. Wang, Z. G. Cui, Q. Q. Li, S. X. Dai and Z. L. Du, Appl Phys Lett, 2012, 100.

57. L. S. Jung, C. T. Campbell, T. M. Chinowsky, M. N. Mar and S. S. Yee, Langmuir, 1998, 14, 5636-5648.

58. P. P. Jia and J. Yang, Appl Phys Lett, 2013, 102. 243107.

59. J. Junesch, T. Sannomiya and A. B. Dahlin, Acs Nano, 2012, 6, 10405-10415.

60. A. G. Brolo, R. Gordon, B. Leathem and K. L. Kavanagh, Langmuir, 2004, 20, 48134815.

61. J. Henzie, M. H. Lee and T. W. Odom, Nat Nanotechnol, 2007, 2, 549-554. 
62. D. Sinton, R. Gordon and A. G. Brolo, Microfluid Nanofluid, 2008, 4, 107-116.

63. C. Escobedo, Lab Chip, 2013, 13, 2445-2463.

64. A. Barik, L. M. Otto, D. Yoo, J. Jose, T. W. Johnson and S. H. Oh, Nano Lett, 2014, 14, 2006-2012.

65. A. De Leebeeck, L. K. S. Kumar, V. de Lange, D. Sinton, R. Gordon and A. G. Brolo, Anal Chem, 2007, 79, 4094-4100.

66. A. Lesuffleur, H. Im, N. C. Lindquist, K. S. Lim and S. H. Oh, P Soc Photo-Opt Ins, $2008,7035$.

67. H. Im, A. Lesuffleur, N. C. Lindquist and S. H. Oh, Anal Chem, 2009, 81, 2854-2859.

68. C. Escobedo, S. Vincent, A. I. K. Choudhury, J. Campbell, A. G. Brolo, D. Sinton and R. Gordon, J Micromech Microeng, 2011, 21.

69. T. Sannomiya, O. Scholder, K. Jefimovs, C. Hafner and A. B. Dahlin, Small, 2011, 7, 1653-1663.

70. J. Ji and D. N. Larson, Abstr Pap Am Chem S, 2008, 235. 2491-8.

71. J. Ji, J. G. O'Connell, D. J. D. Carter and D. N. Larson, Anal Chem, 2008, 80, 2491-2498.

72. A. Lesuffleur, H. Im, N. C. Lindquist and S. H. Oh, Appl Phys Lett, 2007, 90. 243110.

73. J. C. Sharpe, J. S. Mitchell, L. Lin, H. Sedoglavich and R. J. Blaikie, Anal Chem, 2008, 80, 2244-2249. 
74. A. Lesuffleur, H. Im, N. C. Lindquist, K. S. Lim and S. H. Oh, Opt Express, 2008, 16, 219-224.

75. H. Im, J. N. Sutherland, J. A. Maynard and S. H. Oh, Anal Chem, 2012, 84, 1941-1947.

76. H. Im, S. H. Lee, N. J. Wittenberg, T. W. Johnson, N. C. Lindquist, P. Nagpal, D. J. Norris and S. H. Oh, Acs Nano, 2011, 5, 6244-6253.

77. C. Escobedo, A. G. Brolo, R. Gordon and D. Sinton, Proc Spie, 2011, 7929.79290Q.

78. J. Ferreira, M. J. L. Santos, M. M. Rahman, A. G. Brolo, R. Gordon, D. Sinton and E. M. Girotto, J Am Chem Soc, 2009, 131, 436-437.

79. A. Buchenauer, M. Bialon, D. Segun, C. Puttmann, C. Stein, S. Barth and U. Schnakenberg, J Micromech Microeng, 2014, 24. 034001.

80. H. Ko, S. Singamaneni and V. V. Tsukruk, Small, 2008, 4, 1576-1599.

81. M. K. Fan, G. F. S. Andrade and A. G. Brolo, Anal Chim Acta, 2011, 693, 7-25.

82. G. A. Baker and D. S. Moore, Anal Bioanal Chem, 2005, 382, 1751-1770.

83. X. M. Lin, Y. Cui, Y. H. Xu, B. Ren and Z. Q. Tian, Anal Bioanal Chem, 2009, 394, $1729-1745$.

84. T. Itoh, K. Yoshida, H. Tamaru, V. Biju and M. Ishikawa, J Photoch Photobio A, 2011, 219, 167-179.

85. D. Y. Wu, J. F. Li, B. Ren and Z. Q. Tian, Chem Soc Rev, 2008, 37, 1025-1041. 
86. A. Campion, J. E. Ivanecky, C. M. Child and M. Foster, J Am Chem Soc, 1995, 117, 11807-11808.

87. N. Felidj, J. Aubard, G. Levi, J. R. Krenn, A. Hohenau, G. Schider, A. Leitner and F. R. Aussenegg, Appl Phys Lett, 2003, 82, 3095-3097.

88. N. Felidj, J. Aubard, G. Levi, J. R. Krenn, M. Salerno, G. Schider, B. Lamprecht, A. Leitner and F. R. Aussenegg, Phys Rev B, 2002, 65. 075419.

89. A. G. Brolo, E. Arctander, R. Gordon, B. Leathem and K. L. Kavanagh, Nano Lett, 2004, 4, 2015-2018.

90. G. F. S. Andrade, J. G. Hayashi, M. M. Rahman, W. J. Salcedo, C. M. B. Cordeiro and A. G. Brolo, Plasmonics, 2013, 8, 1113-1121.

91. W. S. Yue, Y. Yang, Z. H. Wang, L. Q. Chen and X. B. Wang, J Phys Chem C, 2013, 117, 21908-21915.

92. P. Mandal, A. Nandi and S. A. Ramakrishna, J Appl Phys, 2012, 112. 044314.

93. G. Q. Liu, Y. Li, G. T. Duan, J. J. Wang, C. H. Liang and W. P. Cai, Acs Appl Mater Inter, 2012, 4, 1-5.

94. T. H. Reilly, S. H. Chang, J. D. Corbman, G. C. Schatz and K. L. Rowlen, J Phys Chem C, 2007, 111, 1689-1694.

95. Q. M. Yu, P. Guan, D. Qin, G. Golden and P. M. Wallace, Nano Lett, 2008, 8, 1923-1928. 
96. Q. M. Yu, S. Braswell, B. Christin, J. J. Xu, P. M. Wallace, H. Gong and D. Kaminsky, Nanotechnology, 2010, 21.

97. D. Correia-Ledo, K. F. Gibson, A. Dhawan, M. Couture, T. Vo-Dinh, D. Graham and J. F. Masson, J Phys Chem C, 2012, 116, 6884-6892.

98. J. C. Yang, H. W. Gao, J. Y. Suh, W. Zhou, M. H. Lee and T. W. Odom, Nano Lett, 2010, 10, 3173-3178.

99. B. C. Galarreta, E. Harte, N. Marquestaut, P. R. Norton and F. Lagugne-Labarthet, Phys Chem Chem Phys, 2010, 12, 6810-6816.

100. H. Fischer and O. J. F. Martin, Opt Express, 2008, 16, 9144-9154.

101. S. H. Lee, K. C. Bantz, N. C. Lindquist, S. H. Oh and C. L. Haynes, Langmuir, 2009, 25, 13685-13693.

102. J. T. Li, S. K. Cushing, P. Zheng, F. K. Meng, D. Chu and N. Q. Wu, Nat Commun, 2013, 4. 2561 .

103. E. D. Palik, Handbook of optical constants of solids, Academic Press, Orlando, 1985.

104. W. A. Murray, S. Astilean and W. L. Barnes, Phys Rev B, 2004, 69.

105. M. Li, H. L. Gou, I. Al-Ogaidi and N. Q. Wu, Acs Sustain Chem Eng, 2013, 1, 713-723.

106. Y. Yang, W. Y. Li, H. Qi, Q. F. Zhang, J. Chen, Y. Wang, B. Wang, S. J. Wang and C. Yu, Anal Biochem, 2012, 430, 48-52.

107. Y. H. Lin and W. L. Tseng, Chem Commun, 2009, 6619-6621. 
108. G. Schmalz, K. A. Hiller, P. Garhammer and T. Reitinger, J Dent Res, 2001, 80, 12541254.

109. G. Chakrapani, P. L. Mahanta, D. S. R. Murty and B. Gomathy, Talanta, 2001, 53, 11391147.

110. R. P. Singh and E. R. Pambid, Analyst, 1990, 115, 301-304.

111. M. Krachler, C. Mohl, H. Emons and W. Shotyk, Spectrochim Acta B, 2002, 57, 12771289.

112. S. Dadfarnia, A. M. H. Shabani and M. Gohari, Talanta, 2004, 64, 682-687.

113. Y. Q. Wen, F. F. Xing, S. J. He, S. P. Song, L. H. Wang, Y. T. Long, D. Li and C. H. Fan, Chem Commun, 2010, 46, 2596-2598.

114. J. Hatai, S. Pal and S. Bandyopadhyay, Rsc Adv, 2012, 2, 10941-10947.

115. G. Chen, Y. Wang, M. X. Yang, J. Xu, S. J. Goh, M. Pan and H. Y. Chen, J Am Chem Soc, 2010, 132, 3644-3645.

116. M. A. Ochsenkuhn, P. R. T. Jess, H. Stoquert, K. Dholakia and C. J. Campbell, Acs Nano, 2009, 3, 3613-3621.

117. Y. W. C. Cao, R. C. Jin and C. A. Mirkin, Science, 2002, 297, 1536-1540.

118. D. M. Wilson, S. Hoyt, J. Janata, K. Booksh and L. Obando, Ieee Sens J, 2001, 1, 256274.

119. F. Hao, C. L. Nehl, J. H. Hafner and P. Nordlander, Nano Lett, 2007, 7, 729-732. 
120. W. Ma, M. Z. Sun, L. G. Xu, L. B. Wang, H. Kuang and C. L. Xu, Chem Commun, 2013, 49, 4989-4991.

121. A. Ono, S. Cao, H. Togashi, M. Tashiro, T. Fujimoto, T. Machinami, S. Oda, Y. Miyake, I. Okamoto and Y. Tanaka, Chem Commun, 2008, 4825-4827.

122. M. Li, S. K. Cushing, J. Zhang, J. Lankford, Z. P. Aguilar, D. Ma and N. Wu, Nanotechnology, 2012, 23, 115501.

123. J. V. Gilfrich and L. S. Birks, Anal Chem, 1984, 56, 77-79. 


\section{Peer-reviewed Publication List}

(1) Jiangtian Li; Scott K. Cushing; Peng Zheng; Tess Senty; Fanke Meng; Alan D. Bristow; Ayyakkannu Manivannan; Nianqiang Wu. Solar Hydrogen Generation by a CdS-AuTiO2 Sandwich Nanorod Array Enhanced with Au Nanoparticle as Electron Relay and Plasmonic Photosensitizer. Journal of American Chemistry Society, 2014, 136 (23), 8438-8449.

(2) Israa Ali Zaidan Al-Ogaidi; Honglei Gou; Abdul Kareem Al-kazaz; Zoraida Aguilar; Alice Melconain; Peng Zheng; Nianqiang Wu. Gold@Silica Core-Shell NanoparticleBased Surface-Enhanced Raman Scattering Biosensor for Label-Free Glucose Detection. Analytica Chimica Acta, 811 (2014), 76-80.

(3) Jiangtian Li; Scott K. Cushing; Peng Zheng; Fanke Meng, Deryn Chu and Nianqiang Wu. Plasmon-induced photonic and energy transfer enhancement of solar water splitting by a hematite nanorod array. Nature Communications. 4:2651 doi: 10.1038/ncomms3651 (2013).

(4) Jiangtian Li; Scott K. Cushing; Joesph Bright; Fanke Meng; Tess Senty; Peng Zheng; Alan Bristow; Nianqiang Wu (Nick). Ag@Cu2O Core-Shell Nanoparticles as VisibleLight Plasmonic Photocatalysts. ACS Catalysis, 2013, 3, 47-51.

(5) Peng Zheng, Guowen He, Hengfeng Li. Recent Advances of Polyimide Based Nanocomposite Materials [J]. Materials China, 2011, (12): 40-45. 